2014

\title{
Adaptive Radiation Therapy for Lung Cancer
}

Christian W. Dial

Virginia Commonwealth University

Follow this and additional works at: https://scholarscompass.vcu.edu/etd

Part of the Oncology Commons

() The Author

\section{Downloaded from}

https://scholarscompass.vcu.edu/etd/3579

This Dissertation is brought to you for free and open access by the Graduate School at VCU Scholars Compass. It has been accepted for inclusion in Theses and Dissertations by an authorized administrator of VCU Scholars Compass. For more information, please contact libcompass@vcu.edu. 
(C) Christian Dial

2014

All rights reserved 


\title{
Adaptive Radiation Therapy for Lung Cancer
}

A dissertation submitted to the Virginia Commonwealth University School of Medicine in partial fulfillment of the requirements for the degree of

\author{
Doctor of Philosophy \\ Medical Physics
}

by

Christian Wesley Dial

Advisors: Dr. Geoffrey D. Hugo, Dr. Jeffrey V. Siebers

Virginia Commonwealth University

Richmond, Virginia July 2014 


\section{Dedication}

We are not completely of our own making;

we depend on a storehouse of talent and character from which to build our lives.

To my father, whose love for learning and balanced analysis of the world have influenced me almost as much as his gentleness, humility, and love towards others.

To my mother, for her love, support, and tireless efforts in behalf of our family and all that surround her.

And to my wife Erin, for bearing the final months and hours of this work with understanding, and for her continual optimism, support, and love. 


\section{Acknowledgements}

I would like to thank my advisors Dr. Geoffrey Hugo and Dr. Jeffrey Siebers for their encouragement to pursue a doctoral degree and for their patience and efforts in seeing this work through. Each expressed confidence in my abilities and always made time to answer questions and offer assistance. I am grateful for their influence in my life and for their support throughout this project.

I would also like to acknowledge Dr. Elisabeth Weiss for her assistance in the clinical aspects of this project, and Dr. Vojislav Kecman for his guidance and suggestions.

Lastly, I wish to thank our department secretary Deanna Pace, my classmates, and all others in the department who offered feedback, administrative support, encouragement, and who have taught me along the way. 


\section{Table of contents}

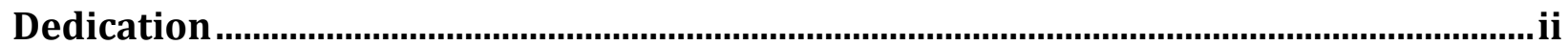

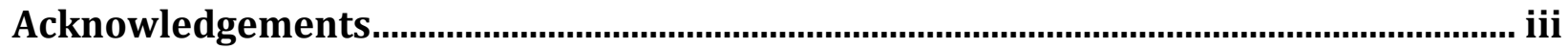

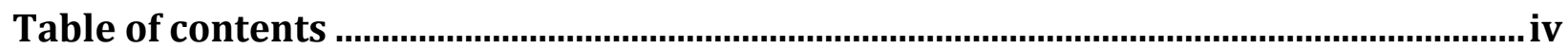

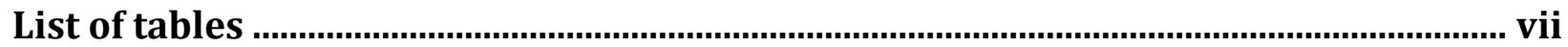

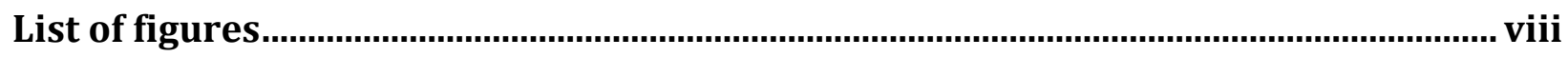

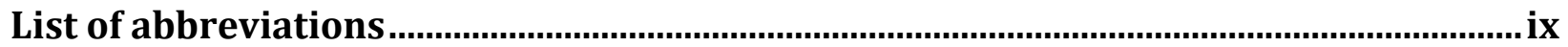

Abstract.................................................................................................................................. X

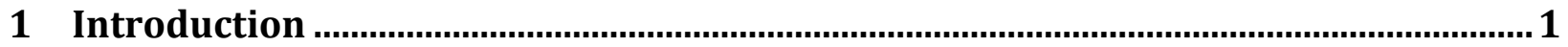

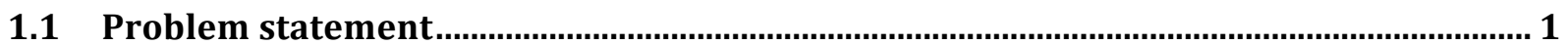

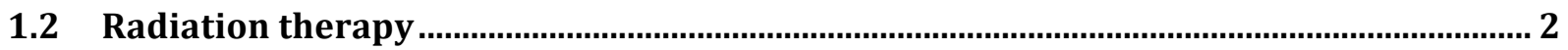

1.2.1 Uncertainties in radiotherapy for lung cancer ........................................................................ 3

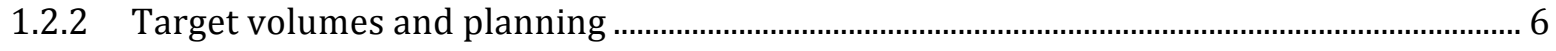

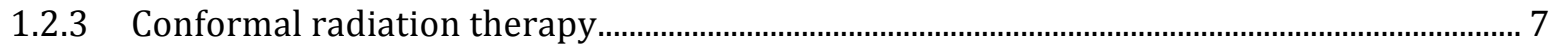

1.2.4 Intensity modulated radiation therapy and inverse planning.................................................... 7

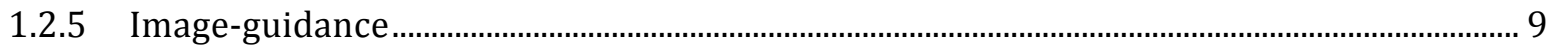

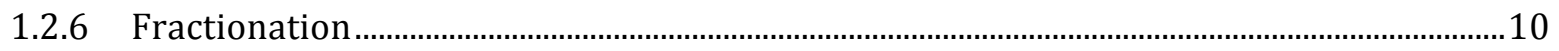

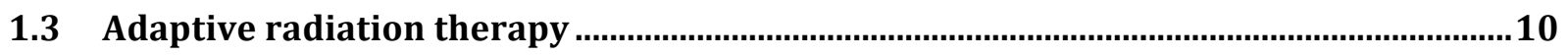

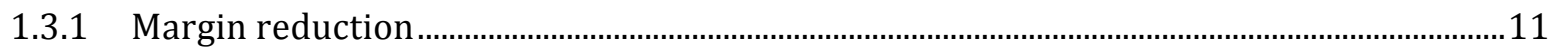




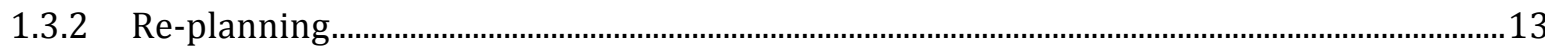

1.4 Purpose and thesis overview

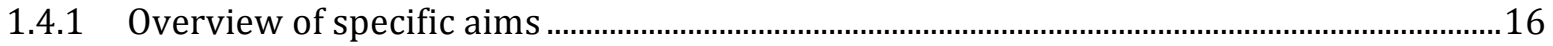

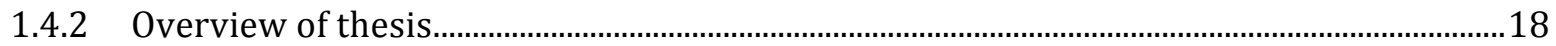

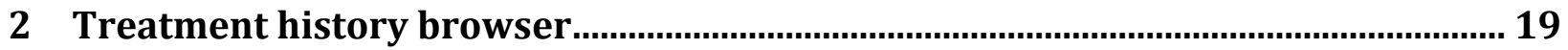

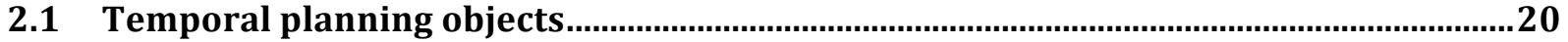

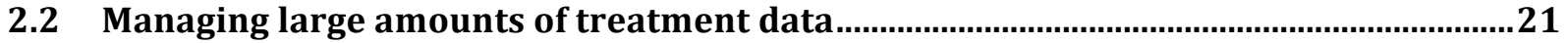

2.3 Methods for evaluating treatments that consist of multiple datasets ...........................22

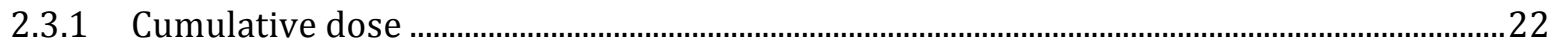

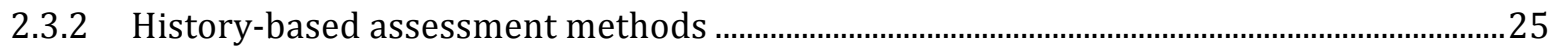

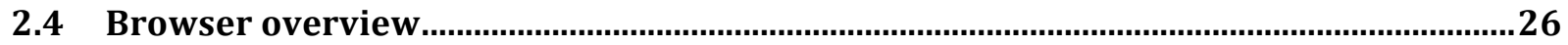

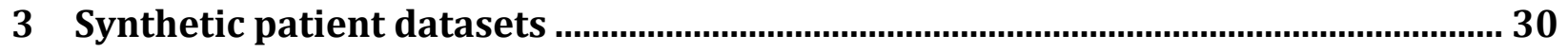

3.1 Statistical modeling of inter-fraction variation using principal component analysis30

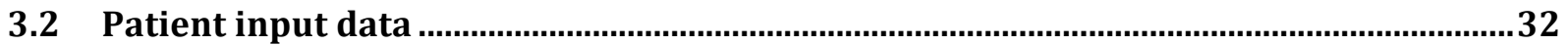

3.2.1 Deformable registration of weekly images .....................................................................................33

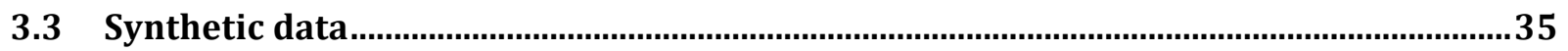

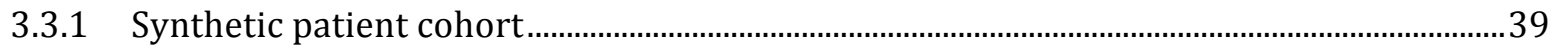

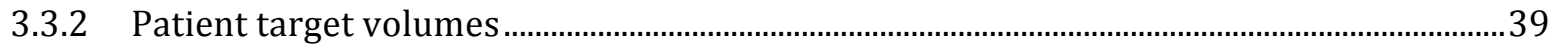

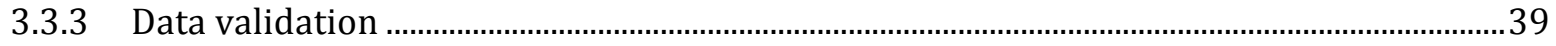

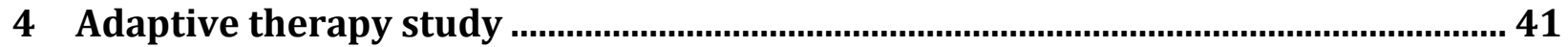

4.1 Correlation of nodal and non-nodal treatment results derived from the same patient 41

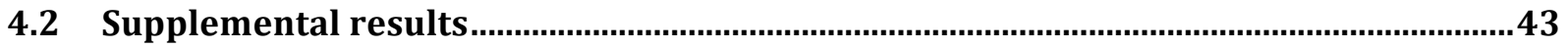

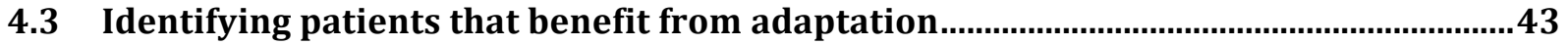


4.4 Differences between planning and simulated-treatment dose distributions..............45

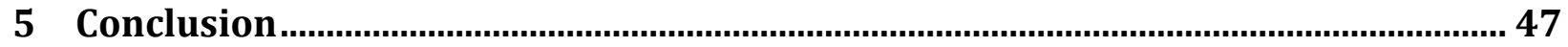

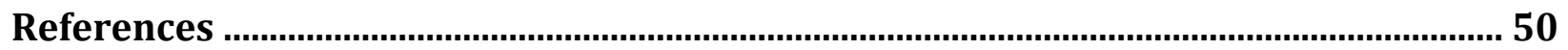

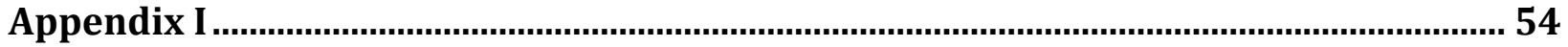

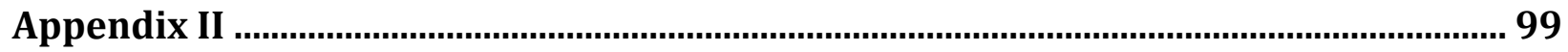

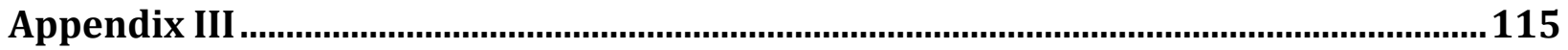

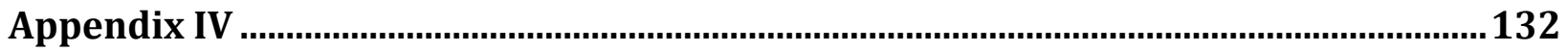

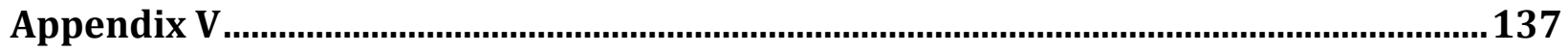

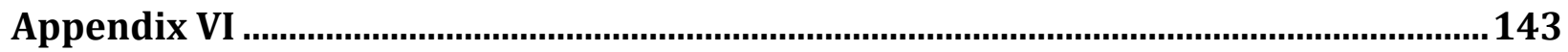

Vita 


\section{List of tables}

Table 3.1. Patient characteristics for the 12 NSCLC patients utilized in the work. $L U L=$ left upper lobe; $L L L=$ left lower lobe; $R U L=$ right upper lobe; $R M L=$ right middle lobe; $R L L=$ right lower lobe 33

Table 3.2. Gross tumor volumes corresponding to the first and last weekly images for a subset of the patient cohort. For the final image, gross tumor volumes are reported for the manually drawn physician contour, the propagated contour using the SICLE algorithm, and the propagated contour using the Demons algorithm. Volume discrepancies between the propagated and manual contours on the final image are included in parentheses. Demons algorithm demonstrated smaller discrepancies between propagated and manual volumes on the final image ( $p=0.02)$. 35

Table 4.1 Mean differences between planning dose distribution and cumulative dose for the no-adapt simulation. Target coverage and normal-tissue dose metrics are included along with mean differences ( $\Delta$ ) and standard deviations $(\sigma)$ for each. Statistical significance as measured by two-tailed paired $t$-tests is indicated in the final column for differences between planned and no-adapt distributions. 


\section{List of figures}

Fig. 3.1 Registration of a temporal series of $n$ images. Registrations are performed between first image in series and all subsequent images to identify corresponding tissue locations in the group of images. (mapping for single point illustrated). 31

Fig. 3.2 Percent gross tumor volumes as a function of treatment week based on physician delineated contours. 33

Fig. 3.3 Synthetic images for patients 1, 3, 4, 5, 6, and 8. Axial slices corresponding to tumor centroid are presented for fractions 1, 18, and 35 (from left to right). 36

Fig. 3.4 Synthetic images for patients 9, 14, 17, 18, 20, and 21. Axial slices corresponding to tumor centroid are presented for fractions 1, 18, and 35 (from left to right). 37

Fig. 3.5 Percent gross tumor volumes for propagated synthetic datasets as a function of treatment fraction. _- 38 Fig. 3.6 Cranial (left) and caudal (right) artifacts in fraction 35 synthetic image for patient 1. 38 Fig. 4.1 Correlation of dose-metric-difference for non-node and nodal simulations derived from the same patient. Plots are devoid of any strong correlation. 42 


\section{List of abbreviations}

ABC: Active breathing control

ART: Adaptive radiation therapy

CBCT: Cone beam computed tomography

CT: Computed tomography

CTV: Clinical target volume

DIR: Deformable image registration

DVF: Displacement vector field

DVH: Dose volume histogram

GTV: Gross tumor volume

ICRU: International Commission on Radiation Units

IMRT: Intensity modulated radiation therapy

LINAC: Linear accelerator

MD: Microscopic disease

MLC: Multi-leaf collimator

MSD: Minimum surface distance

NSCLC: Non-small-cell lung cancer

ODM: Opening density matrix

PCA: Principal component analysis

PTV: Planning target volume

ROI: Region of interest

RTOG: Radiation therapy oncology group

SICLE: Small deformation inverse consistent linear elastic

THB: Treatment history browser

TPS: Treatment planning system

V20: Percent volume receiving at least 20Gy 


\begin{abstract}
ADAPTIVE RADIATION THERAPY FOR LUNG CANCER

By Christian Wesley Dial, Ph.D.

A dissertation submitted to the Virginia Commonwealth University School of Medicine in partial fulfillment of the requirements for the degree of Doctor of Philosophy Medical Physics.
\end{abstract}

Virginia Commonwealth University, 2014

Advisors: Dr. Geoffrey D. Hugo, Assistant Professor; Dr. Jeffrey V. Siebers, Professor Division of Medical Physics Department of Radiation Oncology

Prognosis for lung cancer patients remains poor. For those receiving radiation therapy, local control and survival have been shown to improve with increased doses; however, deliverable dose is often limited by associated toxicity. Therefore, methods that reduce dose to normal tissues and allow isotoxic escalation are desirable. Adaptive radiation therapy seeks to improve treatment by modifying the initial plan throughout delivery, and has been shown to 
decrease normal tissue dose. Studies to date suggest a trend of increasing benefit with increases in replanning frequency; however, replanning is costly in terms of workload and past studies implement at most weekly adaptation. The purpose of this thesis is to quantify the benefit associated with daily replanning and characterize the tradeoff between replanning frequency and adaptive benefit. A software tool is developed to facilitate planning studies and to introduce complimentary methods for evaluating adaptive treatments. Synthetic images and contours are generated for each fraction of a typical fractionation schedule using principal component analysis and a novel method of sampling coefficients that preserves temporal trends in the data (e.g. tumor regression). Using the synthetic datasets, a series of adaptive schedules ranging from no adaption to daily replanning are simulated and compared to quantify adaptive benefits and characterize tradeoffs with frequency. Daily replanning resulted in significant reductions in all normal tissue planning metrics when compared to no adaptation, and incremental reductions were observed with each increase in replanning frequency while the magnitude of average reductions decreased with each step. Modest correlation between absolute change in planning target volume over the course of treatment and reductions in both mean lung dose and mean esophageal dose were observed. 


\section{Introduction}

\subsection{Problem statement}

Cancer ranks second, behind heart disease, among the leading causes of death in the United States for both men and women by a margin of less than $2 \%$. In 2009 , the most recent year for which data is available, 567,628 cancer deaths were reported, accounting for $23.3 \%$ of all mortalities.$^{[1]}$ For 2013, The American Cancer Society estimated 580,350 cancer-related deaths and projected 228,190 of those to be associated with malignancies of the lung, making lung cancer the number one cause of cancer-related death in the United States. ${ }^{[2]}$

Treatment depends on type, site, and stage of disease, and may be influenced by patient specific preferences or comorbidities, but generally includes surgical resection, chemotherapy, radiotherapy, or a combination of the three. In the case of lung cancer, radiotherapy is indicated in early stage disease where comorbidities or patient refusal preclude a surgical approach, and in advanced cases where tumor is unresectable, making it the treatment of choice for approximately $40 \%$ of newly diagnosed lung cancer patients. ${ }^{[3]}$

Prognosis for those diagnosed with lung cancer remain poor; in their most recent report, The National Cancer Institute reports a 5 year survival rate of $16.6 \%$ for those with cancers of the lung. ${ }^{[4]}$ Low survival rates for the large portion of patients diagnosed with lung cancer coupled with the fact that $40 \%$ receive radiation therapy as the primary treatment modality make improvements in radiation therapy for lung cancer an important topic of research.

For patients receiving radiation therapy, local tumor control and survival have been shown to improve with increased doses; ${ }^{[5,6]}$ however, for most patients deliverable dose is limited by 
associated normal tissue toxicity. Reducing normal tissue dose for a given treatment regimen is a common theme in radiotherapy research and allows tumor doses to be escalated until an isotoxic effect is reached for the limiting normal structure.

Advancements in the field that have lead to increased sparing of normal tissues include: conformal radiotherapy, intensity modulated radiation therapy (IMRT), and tools that reduce uncertainties associated with treatment and setup (e.g. image guidance). Adaptive radiation therapy (ART), which is the subject of this thesis, utilizes these techniques in a dynamic approach to accommodate interfraction variation and may be utilized to further reduce exposure of relevant risk structures.

A basic introduction to traditional radiation therapy, ART, and relevant advancements in the field are presented in this section as context for the work presented in later chapters.

\subsection{Radiation therapy}

Radiation therapy seeks to eradicate diseased tissue through the targeted administration of ionizing radiation while minimizing effects on surrounding normal tissues. Biological effects of ionizing radiation include disruption of cellular DNA, resulting in mutations that may compromise cellular function or ultimately prove fatal for the cell. Damage may result from direct action on the DNA, or indirectly through the production of free-radicals - principally, highly reactive oxygen species - which may subsequently lyse base pair bonds. Irreparable damage may signal programmed cell death (apoptosis) or may inhibit cellular division (mitotic death) and thereby end the life of the cell.

The type of radiation utilized may be either gamma-rays or particulate radiation (e.g. electrons) of various energies and is administered via a beam that originates outside of the patient (external-beam radiotherapy) or by placing radioactive sources inside or next to the 
treatment site (brachytherapy). For lung cancer, treatment usually involves external-beam radiotherapy that delivers gamma-rays in the megavoltage range.

Common to all modalities of radiation therapy is the specification of robust target volumes to ensure tumor coverage with a high degree of probability. In order to specify robust target volumes, geometric uncertainties inherent in the planning process must be appreciated and accounted for. A description of the uncertainties associated with radiotherapy for lung cancer are given below.

\subsubsection{Uncertainties in radiotherapy for lung cancer}

The various uncertainties associated with specifying and localizing radiotherapy targets have traditionally been managed through the addition of safety margins to account for intra- and inter-fraction variations. Specifically, uncertainties are related to: identifying and delineating tumor volumes as seen on planning CTs, ascertaining the extent of microscopic invasion and nodal involvement, patient positioning, and motion of target and surrounding anatomy. Anatomical change throughout treatment presents another challenge to accurately localizing structures of interest and may include: tumor regression, patient weight variations, and the development or resolution of local pathology (e.g. atelectasis, pleural effusion, etc.).

\subsubsection{Identifying and delineating gross tumor}

After acquisition of a diagnostic CT scan obtained with the patient in the intended treatment position, organs at risk and gross tumor are outlined by a physician using treatment planning software. While tools exist to aid in segmentation, ultimately contours are based on clinical judgment and have been shown to vary significantly for different observers. In one study, inter-observer variability as defined by the standard deviation of observer contour distances from a common region of interest (voxels designated as gross tumor by at least $50 \%$ of observers) was 
$1.02 \mathrm{~cm}$ for lung lesions when based on diagnostic CT alone. This improved to $0.42 \mathrm{~cm}$ in a second phase of the study that incorporated FDG-PET to help distinguish gross tumor; however, variability of this magnitude still constitutes a significant uncertainty in radiotherapy. ${ }^{[7]}$

Discrepancies at this stage of planning represent systematic errors that are propagated throughout treatment and for some patients may be the dominant source of uncertainty. Causes of variability may include lack of knowledge, inconsistencies in methodology, and lack of contrast between gross tumor and surrounding normal structures or pathology (e.g. atelectasis). ${ }^{[8]}$

\subsubsection{Extent of microscopic disease}

By definition, the gross tumor volume delineated by the physician includes macroscopic disease; however, surrounding tissue may harbor cancerous cells that are not clinically detectable. The extent of subclinical disease represents an additional uncertainty in the planning process that is managed by adding a margin around the grossly detectable tumor. Margins are based on histological evaluations of excised tumors and depend on, among other things, the type and grade of cancer. In a study conducted by Giraud et al., microscopic extension from the gross tumor border for lung cancer ranged from 0.0 to $12.0 \mathrm{~mm}$. For patients included in the study, 8 and 6 $\mathrm{mm}$ accounted for $95 \%$ of the observed microscopic disease in adeno- and squamous-cell carcinomas respectively. ${ }^{[9]}$

\subsubsection{Setup}

After regions of interest have been delineated and a plan developed, the patient is positioned for daily treatments with focus on reproducing the planning geometry. A reference point within the patient is designated when the planning CT is first acquired, and marks are made on the patient's skin for positioning in the coordinate system of the LINAC using in-room lasers. Relative motion of the skin and internal anatomy, the precision with which skin marks are made, 
and the care with which marks are aligned, all contribute to setup error which is composed of both systematic and random components. Though errors vary depending on institution, and from patient to patient, an estimation of $2-4 \mathrm{~mm}$ has been reported for lung cancer. ${ }^{[10]}$

\subsubsection{Respiratory motion and management}

The effects of respiration on tumor motion are complex and vary widely from patient to patient in magnitude, period, and trajectory, thus patient-specific analysis and management are recommended. ${ }^{[11]}$ In a study conducted by Stevens et al., tumor motion due to respiration in the superior-inferior direction ranged from $0.0-2.2 \mathrm{~cm},{ }^{[12]}$ thus respiratory motion represents a significant uncertainty in radiation therapy. In the planning process, respiratory motion can introduce image artifact in the simulation CT leading to systematic errors in both delineation and dose calculation. During treatment, inter- and intra-fraction motion may cause further deviations in dose from the planned distribution.

Motion management may include: additional target margins to encompass the range of motion throughout treatment; gating using a surrogate for tumor location (e.g. abdominal surface) to restrict beam-on time to a particular phase of motion; breath-hold using a spirometer and other forms of bio-feedback to isolate the tumor in a given location; forced shallow breathing with abdominal compression to limit movement; or tumor-tracking in which the beam position is dynamically modified. Each method warrants separate analysis and appropriate margins for residual errors.

\subsubsection{Tumor regression}

Regression of the gross tumor volume throughout the course of radiotherapy has been observed by various authors. Kupelian was one of the first to quantify this change in non-smallcell lung cancer (NSCLC) reporting an average volume decrease of $1.2 \%(0.6-2.3 \%)$ per day. ${ }^{[13]}$ 
Britton et al. acquired weekly 4DCT datasets for 8 NSCLC patients receiving definitive radiotherapy and found a median decrease of $41.7 \%(8.1-71.3 \%) .{ }^{[14]}$ Finally, van Zwienen et al. performed a more extensive study consisting of 114 lung cancer patients with weekly cone-beam CTs (CBCT), 46 (40\%) demonstrated tumor regression with an average volume change of $37 \%$ over the course of treatment. ${ }^{[15]}$

\subsubsection{Patho-anatomical change}

The development or resolution of local pathology throughout treatment may dramatically affect the anatomy of a given region and significantly alter planned dose distributions. In lung cancer, two types of patho-anatomical change are of interest. 1) Atelectasis is a collapse of lung parenchyma that may be due to obstruction of a main airway. Affected regions often have similar CT appearance to gross tumor which may make it difficult to distinguish between the two.

2) Pleural effusion is an accumulation of excess fluid in the space between the visceral and parietal pleura that surround the lungs, compressing and displacing adjacent regions.

\subsubsection{Target volumes and planning}

Various volumes are specified in the planning process to effectively target diseased regions. International Commission on Radiation Units (ICRU) report $50^{[16]}$ formalizes target volume definitions and recommends the following volumes be specified: the gross tumor volume (GTV), which consists of grossly detectable disease; the clinical target volume (CTV), which is an appropriate expansion of the GTV to incorporate potential subclinical disease; and the planning target volume (PTV), which is an expansion of the CTV to account for residual errors in setup and motion. While GTV and CTV specify regions of anatomy that presumably harbor disease, the PTV is a region of space that exists in the coordinate system of the linear accelerator 
(LINAC) specified to ensure a high probability of coverage in the presence of geometric uncertainty.

After delineation of target volumes and risk structures on the CT image, planning is carried out using treatment planning software to specify a number of beams and calculate the resulting dose distribution within the patient. Appropriate beam angles help avoid excessive irradiation of risk structures along with collimation to limit the extents of the beam to PTV.

\subsubsection{Conformal radiation therapy}

Conformal therapy is a technique that further modifies the beam to conform to the shape of the PTV as opposed to a rectangular field that is limited to the extents of the target volume. Beam shaping is achieved by inserting a custom cutout in the path of the beam that conforms to the $2 \mathrm{D}$ projection of the target volume as observed through the beams-eye-view or by using a multi-leaf collimator (MLC) which is mounted in the gantry of the linear accelerator. The MLC consists of multiple leaf pairs that are manufactured of a high- $\mathrm{Z}$ material and can be inserted into or retracted from the path of the beam automatically to create custom apertures.

\subsubsection{Intensity modulated radiation therapy and inverse planning}

While beam shaping increases dose conformality, certain distributions may remain unachievable (e.g. concave dose distributions). In intensity modulated radiation therapy (IMRT), non-uniform fluence patterns for a given beam angle and aperture are delivered; practically this is achieved by delivering multiple smaller beam segments (beamlets) to build up a fluence profile. The superposition of multiple non-uniform fluence profiles allows a greater degree of flexibility in achievable iso-dose shapes, and may increase dose gradients and conformality, but 
also increases treatment complexity. The number of plan-design parameters are significantly increased in IMRT making manual parameter selection and design impractical.

Computer assisted optimization of plan parameters is an essential element of modern IMRT. Treatment intent is translated into $n$ mathematical objectives $F_{k}, k=1 \ldots n$, that make up a composite cost function $F(\tau)$, where $\tau$ is a set of planning parameters to be optimized.

$$
F(\tau)=\sum_{k=1}^{n} F_{k}
$$

Objective functions may be based on physical expressions of dose or more complicated biological models of treatment effect. Additionally, a set of constraints $C(\tau)$ may also be specified and the optimization seeks the solution:

$$
\begin{aligned}
& \min _{\tau} F(\tau), \\
& C(\tau) \leq 0
\end{aligned}
$$

In this work, IMRT optimizations utilize dose volume criteria, and objective functions are of the general form:

$$
F_{k}=w_{k} \sum_{i \in V}\left(\frac{d_{i}-d_{k}}{d_{k}}\right)^{2} \Delta v_{i}
$$

Where $w_{k}$ is an importance weighting factor, $V$ is the volume of interest, $d_{i}$ is the current dose for voxel $i, d_{k}$ is the intended dose for voxel $i$, and $\Delta v_{i}$ is the fraction of volume $V$ represented by voxel $i$. Weighting factors are specified by the planner and significantly affect resulting plans.

The optimizer begins with an initial estimate of the discrete intensity map of beamlets, or opening density matrix (ODM), and iteratively seeks a solution. For multi-modal cost functions, 
solutions may converge to sub-optimal local minima depending on initial approximations and the search algorithm employed.

\subsubsection{Image-guidance}

The use of onboard imaging devices is an important advancement in modern radiation therapy. Images acquired on the day of treatment and in the treatment geometry provide up-todate positional information on target and normal tissue structures, allowing deviations from the planning geometry to be detected and corrected for. Increases in positional accuracy justify commensurate reductions in safety margins, which decreases dose to surrounding tissue, and potentially increases the therapeutic ratio of treatment.

The frequency with which image-guidance is utilized is important in determining safety margin reductions. Image-guidance strategies that are implemented periodically throughout treatment must allow for residual interfraction errors; when daily-guidance is utilized, safety margins need not include such considerations.

The effect of using daily image-guidance on safety margins has been reported in the literature by various authors. Yeung et al. commented on the significance of cone-beam CT in fractionated radiotherapy for lung, reporting a reduction of approximately $1.5 \mathrm{~cm}$ in PTV margin when compared to alignment protocols that relied on surface marks alone. ${ }^{[17]}$ In another study, Bissonnette et al. calculated population based margins assuming daily CBCT and remotecontrolled couch adjustments to setup the patient; they concluded that post-correction setup margins should be on the order of 2 to $3 \mathrm{~mm} .^{[18]}$

Safety margins utilized in this work were based on a protocol produced by the Radiation Therapy Oncology Group (RTOG) assuming daily image-guidance, and are consistent with those suggested by Bissonnette. 


\subsubsection{Fractionation}

In addition to conforming dose to target structures, fractionation (i.e. administering multiple fractions of prescribed dose over a period of time) maximizes the therapeutic ratio of treatment by capitalizing on inherent differences in radiosensitivity of clonogenic and nonclonogenic cellular populations. Briefly, tissue specific dynamics related to repair of damage, repopulation of cells, oxygenation of tissues, and assortment into radiosensitive stages of the cellular cycle, dictate differences in irradiated tissue response. Repair of sub-lethal damage in normal tissues exceed that in the tumor, thus fractionation spares normal tissues while still eradicating clonogenic populations. In addition to repair, repopulation of normal-tissue cells in the time between treatments enhances the sparing effect of fractionation. For the tumor, fractionation increases free-radical production as oxygen is restored, and allows redistribution of cells into radiosensitive stages of the cell cycle, both of which increase the effectiveness of the delivered dose.

\subsection{Adaptive radiation therapy}

Traditionally, a single arrangement of beams and apertures based on an initial planning image are delivered throughout the course of treatment. Plans are created to be robust against variation and other uncertainty through the addition of safety margins to the primary target volumes.

Adaptive radiation therapy (ART) was first introduced by Yan et al. ${ }^{[19]}$ and was defined as a feedback loop that incorporates measurements of treatment variation in modifying and reoptimizing the treatment plan, as opposed to a fixed plan approach. The early work of Yan focused on mitigating geometrical uncertainty as a means to escalate dose. ${ }^{[20,21]}$ However, in its most general sense, ART may incorporate any additional measurement in improving the different 


\section{Introduction}

\subsection{Problem statement}

Cancer ranks second, behind heart disease, among the leading causes of death in the United States for both men and women by a margin of less than $2 \%$. In 2009 , the most recent year for which data is available, 567,628 cancer deaths were reported, accounting for $23.3 \%$ of all mortalities.$^{[1]}$ For 2013, The American Cancer Society estimated 580,350 cancer-related deaths and projected 228,190 of those to be associated with malignancies of the lung, making lung cancer the number one cause of cancer-related death in the United States. ${ }^{[2]}$

Treatment depends on type, site, and stage of disease, and may be influenced by patient specific preferences or comorbidities, but generally includes surgical resection, chemotherapy, radiotherapy, or a combination of the three. In the case of lung cancer, radiotherapy is indicated in early stage disease where comorbidities or patient refusal preclude a surgical approach, and in advanced cases where tumor is unresectable, making it the treatment of choice for approximately $40 \%$ of newly diagnosed lung cancer patients. ${ }^{[3]}$

Prognosis for those diagnosed with lung cancer remain poor; in their most recent report, The National Cancer Institute reports a 5 year survival rate of $16.6 \%$ for those with cancers of the lung. ${ }^{[4]}$ Low survival rates for the large portion of patients diagnosed with lung cancer coupled with the fact that $40 \%$ receive radiation therapy as the primary treatment modality make improvements in radiation therapy for lung cancer an important topic of research.

For patients receiving radiation therapy, local tumor control and survival have been shown to improve with increased doses; ${ }^{[5,6]}$ however, for most patients deliverable dose is limited by 
associated normal tissue toxicity. Reducing normal tissue dose for a given treatment regimen is a common theme in radiotherapy research and allows tumor doses to be escalated until an isotoxic effect is reached for the limiting normal structure.

Advancements in the field that have lead to increased sparing of normal tissues include: conformal radiotherapy, intensity modulated radiation therapy (IMRT), and tools that reduce uncertainties associated with treatment and setup (e.g. image guidance). Adaptive radiation therapy (ART), which is the subject of this thesis, utilizes these techniques in a dynamic approach to accommodate interfraction variation and may be utilized to further reduce exposure of relevant risk structures.

A basic introduction to traditional radiation therapy, ART, and relevant advancements in the field are presented in this section as context for the work presented in later chapters.

\subsection{Radiation therapy}

Radiation therapy seeks to eradicate diseased tissue through the targeted administration of ionizing radiation while minimizing effects on surrounding normal tissues. Biological effects of ionizing radiation include disruption of cellular DNA, resulting in mutations that may compromise cellular function or ultimately prove fatal for the cell. Damage may result from direct action on the DNA, or indirectly through the production of free-radicals - principally, highly reactive oxygen species - which may subsequently lyse base pair bonds. Irreparable damage may signal programmed cell death (apoptosis) or may inhibit cellular division (mitotic death) and thereby end the life of the cell.

The type of radiation utilized may be either gamma-rays or particulate radiation (e.g. electrons) of various energies and is administered via a beam that originates outside of the patient (external-beam radiotherapy) or by placing radioactive sources inside or next to the 
treatment site (brachytherapy). For lung cancer, treatment usually involves external-beam radiotherapy that delivers gamma-rays in the megavoltage range.

Common to all modalities of radiation therapy is the specification of robust target volumes to ensure tumor coverage with a high degree of probability. In order to specify robust target volumes, geometric uncertainties inherent in the planning process must be appreciated and accounted for. A description of the uncertainties associated with radiotherapy for lung cancer are given below.

\subsubsection{Uncertainties in radiotherapy for lung cancer}

The various uncertainties associated with specifying and localizing radiotherapy targets have traditionally been managed through the addition of safety margins to account for intra- and inter-fraction variations. Specifically, uncertainties are related to: identifying and delineating tumor volumes as seen on planning CTs, ascertaining the extent of microscopic invasion and nodal involvement, patient positioning, and motion of target and surrounding anatomy. Anatomical change throughout treatment presents another challenge to accurately localizing structures of interest and may include: tumor regression, patient weight variations, and the development or resolution of local pathology (e.g. atelectasis, pleural effusion, etc.).

\subsubsection{Identifying and delineating gross tumor}

After acquisition of a diagnostic CT scan obtained with the patient in the intended treatment position, organs at risk and gross tumor are outlined by a physician using treatment planning software. While tools exist to aid in segmentation, ultimately contours are based on clinical judgment and have been shown to vary significantly for different observers. In one study, inter-observer variability as defined by the standard deviation of observer contour distances from a common region of interest (voxels designated as gross tumor by at least $50 \%$ of observers) was 
$1.02 \mathrm{~cm}$ for lung lesions when based on diagnostic CT alone. This improved to $0.42 \mathrm{~cm}$ in a second phase of the study that incorporated FDG-PET to help distinguish gross tumor; however, variability of this magnitude still constitutes a significant uncertainty in radiotherapy. ${ }^{[7]}$

Discrepancies at this stage of planning represent systematic errors that are propagated throughout treatment and for some patients may be the dominant source of uncertainty. Causes of variability may include lack of knowledge, inconsistencies in methodology, and lack of contrast between gross tumor and surrounding normal structures or pathology (e.g. atelectasis). ${ }^{[8]}$

\subsubsection{Extent of microscopic disease}

By definition, the gross tumor volume delineated by the physician includes macroscopic disease; however, surrounding tissue may harbor cancerous cells that are not clinically detectable. The extent of subclinical disease represents an additional uncertainty in the planning process that is managed by adding a margin around the grossly detectable tumor. Margins are based on histological evaluations of excised tumors and depend on, among other things, the type and grade of cancer. In a study conducted by Giraud et al., microscopic extension from the gross tumor border for lung cancer ranged from 0.0 to $12.0 \mathrm{~mm}$. For patients included in the study, 8 and 6 $\mathrm{mm}$ accounted for $95 \%$ of the observed microscopic disease in adeno- and squamous-cell carcinomas respectively. ${ }^{[9]}$

\subsubsection{Setup}

After regions of interest have been delineated and a plan developed, the patient is positioned for daily treatments with focus on reproducing the planning geometry. A reference point within the patient is designated when the planning CT is first acquired, and marks are made on the patient's skin for positioning in the coordinate system of the LINAC using in-room lasers. Relative motion of the skin and internal anatomy, the precision with which skin marks are made, 
and the care with which marks are aligned, all contribute to setup error which is composed of both systematic and random components. Though errors vary depending on institution, and from patient to patient, an estimation of $2-4 \mathrm{~mm}$ has been reported for lung cancer. ${ }^{[10]}$

\subsubsection{Respiratory motion and management}

The effects of respiration on tumor motion are complex and vary widely from patient to patient in magnitude, period, and trajectory, thus patient-specific analysis and management are recommended. ${ }^{[11]}$ In a study conducted by Stevens et al., tumor motion due to respiration in the superior-inferior direction ranged from $0.0-2.2 \mathrm{~cm},{ }^{[12]}$ thus respiratory motion represents a significant uncertainty in radiation therapy. In the planning process, respiratory motion can introduce image artifact in the simulation CT leading to systematic errors in both delineation and dose calculation. During treatment, inter- and intra-fraction motion may cause further deviations in dose from the planned distribution.

Motion management may include: additional target margins to encompass the range of motion throughout treatment; gating using a surrogate for tumor location (e.g. abdominal surface) to restrict beam-on time to a particular phase of motion; breath-hold using a spirometer and other forms of bio-feedback to isolate the tumor in a given location; forced shallow breathing with abdominal compression to limit movement; or tumor-tracking in which the beam position is dynamically modified. Each method warrants separate analysis and appropriate margins for residual errors.

\subsubsection{Tumor regression}

Regression of the gross tumor volume throughout the course of radiotherapy has been observed by various authors. Kupelian was one of the first to quantify this change in non-smallcell lung cancer (NSCLC) reporting an average volume decrease of $1.2 \%(0.6-2.3 \%)$ per day. ${ }^{[13]}$ 
Britton et al. acquired weekly 4DCT datasets for 8 NSCLC patients receiving definitive radiotherapy and found a median decrease of $41.7 \%(8.1-71.3 \%) .{ }^{[14]}$ Finally, van Zwienen et al. performed a more extensive study consisting of 114 lung cancer patients with weekly cone-beam CTs (CBCT), 46 (40\%) demonstrated tumor regression with an average volume change of $37 \%$ over the course of treatment. ${ }^{[15]}$

\subsubsection{Patho-anatomical change}

The development or resolution of local pathology throughout treatment may dramatically affect the anatomy of a given region and significantly alter planned dose distributions. In lung cancer, two types of patho-anatomical change are of interest. 1) Atelectasis is a collapse of lung parenchyma that may be due to obstruction of a main airway. Affected regions often have similar CT appearance to gross tumor which may make it difficult to distinguish between the two.

2) Pleural effusion is an accumulation of excess fluid in the space between the visceral and parietal pleura that surround the lungs, compressing and displacing adjacent regions.

\subsubsection{Target volumes and planning}

Various volumes are specified in the planning process to effectively target diseased regions. International Commission on Radiation Units (ICRU) report $50^{[16]}$ formalizes target volume definitions and recommends the following volumes be specified: the gross tumor volume (GTV), which consists of grossly detectable disease; the clinical target volume (CTV), which is an appropriate expansion of the GTV to incorporate potential subclinical disease; and the planning target volume (PTV), which is an expansion of the CTV to account for residual errors in setup and motion. While GTV and CTV specify regions of anatomy that presumably harbor disease, the PTV is a region of space that exists in the coordinate system of the linear accelerator 
(LINAC) specified to ensure a high probability of coverage in the presence of geometric uncertainty.

After delineation of target volumes and risk structures on the CT image, planning is carried out using treatment planning software to specify a number of beams and calculate the resulting dose distribution within the patient. Appropriate beam angles help avoid excessive irradiation of risk structures along with collimation to limit the extents of the beam to PTV.

\subsubsection{Conformal radiation therapy}

Conformal therapy is a technique that further modifies the beam to conform to the shape of the PTV as opposed to a rectangular field that is limited to the extents of the target volume. Beam shaping is achieved by inserting a custom cutout in the path of the beam that conforms to the $2 \mathrm{D}$ projection of the target volume as observed through the beams-eye-view or by using a multi-leaf collimator (MLC) which is mounted in the gantry of the linear accelerator. The MLC consists of multiple leaf pairs that are manufactured of a high- $\mathrm{Z}$ material and can be inserted into or retracted from the path of the beam automatically to create custom apertures.

\subsubsection{Intensity modulated radiation therapy and inverse planning}

While beam shaping increases dose conformality, certain distributions may remain unachievable (e.g. concave dose distributions). In intensity modulated radiation therapy (IMRT), non-uniform fluence patterns for a given beam angle and aperture are delivered; practically this is achieved by delivering multiple smaller beam segments (beamlets) to build up a fluence profile. The superposition of multiple non-uniform fluence profiles allows a greater degree of flexibility in achievable iso-dose shapes, and may increase dose gradients and conformality, but 
also increases treatment complexity. The number of plan-design parameters are significantly increased in IMRT making manual parameter selection and design impractical.

Computer assisted optimization of plan parameters is an essential element of modern IMRT. Treatment intent is translated into $n$ mathematical objectives $F_{k}, k=1 \ldots n$, that make up a composite cost function $F(\tau)$, where $\tau$ is a set of planning parameters to be optimized.

$$
F(\tau)=\sum_{k=1}^{n} F_{k}
$$

Objective functions may be based on physical expressions of dose or more complicated biological models of treatment effect. Additionally, a set of constraints $C(\tau)$ may also be specified and the optimization seeks the solution:

$$
\begin{aligned}
& \min _{\tau} F(\tau), \\
& C(\tau) \leq 0
\end{aligned}
$$

In this work, IMRT optimizations utilize dose volume criteria, and objective functions are of the general form:

$$
F_{k}=w_{k} \sum_{i \in V}\left(\frac{d_{i}-d_{k}}{d_{k}}\right)^{2} \Delta v_{i}
$$

Where $w_{k}$ is an importance weighting factor, $V$ is the volume of interest, $d_{i}$ is the current dose for voxel $i, d_{k}$ is the intended dose for voxel $i$, and $\Delta v_{i}$ is the fraction of volume $V$ represented by voxel $i$. Weighting factors are specified by the planner and significantly affect resulting plans.

The optimizer begins with an initial estimate of the discrete intensity map of beamlets, or opening density matrix (ODM), and iteratively seeks a solution. For multi-modal cost functions, 
solutions may converge to sub-optimal local minima depending on initial approximations and the search algorithm employed.

\subsubsection{Image-guidance}

The use of onboard imaging devices is an important advancement in modern radiation therapy. Images acquired on the day of treatment and in the treatment geometry provide up-todate positional information on target and normal tissue structures, allowing deviations from the planning geometry to be detected and corrected for. Increases in positional accuracy justify commensurate reductions in safety margins, which decreases dose to surrounding tissue, and potentially increases the therapeutic ratio of treatment.

The frequency with which image-guidance is utilized is important in determining safety margin reductions. Image-guidance strategies that are implemented periodically throughout treatment must allow for residual interfraction errors; when daily-guidance is utilized, safety margins need not include such considerations.

The effect of using daily image-guidance on safety margins has been reported in the literature by various authors. Yeung et al. commented on the significance of cone-beam CT in fractionated radiotherapy for lung, reporting a reduction of approximately $1.5 \mathrm{~cm}$ in PTV margin when compared to alignment protocols that relied on surface marks alone. ${ }^{[17]}$ In another study, Bissonnette et al. calculated population based margins assuming daily CBCT and remotecontrolled couch adjustments to setup the patient; they concluded that post-correction setup margins should be on the order of 2 to $3 \mathrm{~mm} .^{[18]}$

Safety margins utilized in this work were based on a protocol produced by the Radiation Therapy Oncology Group (RTOG) assuming daily image-guidance, and are consistent with those suggested by Bissonnette. 


\subsubsection{Fractionation}

In addition to conforming dose to target structures, fractionation (i.e. administering multiple fractions of prescribed dose over a period of time) maximizes the therapeutic ratio of treatment by capitalizing on inherent differences in radiosensitivity of clonogenic and nonclonogenic cellular populations. Briefly, tissue specific dynamics related to repair of damage, repopulation of cells, oxygenation of tissues, and assortment into radiosensitive stages of the cellular cycle, dictate differences in irradiated tissue response. Repair of sub-lethal damage in normal tissues exceed that in the tumor, thus fractionation spares normal tissues while still eradicating clonogenic populations. In addition to repair, repopulation of normal-tissue cells in the time between treatments enhances the sparing effect of fractionation. For the tumor, fractionation increases free-radical production as oxygen is restored, and allows redistribution of cells into radiosensitive stages of the cell cycle, both of which increase the effectiveness of the delivered dose.

\subsection{Adaptive radiation therapy}

Traditionally, a single arrangement of beams and apertures based on an initial planning image are delivered throughout the course of treatment. Plans are created to be robust against variation and other uncertainty through the addition of safety margins to the primary target volumes.

Adaptive radiation therapy (ART) was first introduced by Yan et al. ${ }^{[19]}$ and was defined as a feedback loop that incorporates measurements of treatment variation in modifying and reoptimizing the treatment plan, as opposed to a fixed plan approach. The early work of Yan focused on mitigating geometrical uncertainty as a means to escalate dose. ${ }^{[20,21]}$ However, in its most general sense, ART may incorporate any additional measurement in improving the different 


\begin{abstract}
ADAPTIVE RADIATION THERAPY FOR LUNG CANCER

Christian Wesley Dial

A dissertation submitted to the Virginia Commonwealth University School of Medicine in partial fulfillment of the requirements for the degree of Doctor of Philosophy Medical Physics.
\end{abstract}

Virginia Commonwealth University, 2014

Advisors: Dr. Geoffrey D. Hugo, Assistant Professor; Dr. Jeffrey V. Siebers, Professor Division of Medical Physics

Department of Radiation Oncology

Prognosis for lung cancer patients remains poor. For those receiving radiation therapy, local control and survival have been shown to improve with increased doses; however, deliverable dose is often limited by associated toxicity. Therefore, methods that reduce dose to normal tissues and allow isotoxic escalation are desirable. Adaptive radiation therapy seeks to 
improve treatment by modifying the initial plan throughout delivery, and has been shown to decrease normal tissue dose. Studies to date suggest a trend of increasing benefit with increases in replanning frequency; however, replanning is costly in terms of workload and past studies implement at most weekly adaptation. The purpose of this thesis is to quantify the benefit associated with daily replanning and characterize the tradeoff between replanning frequency and adaptive benefit. A software tool is developed to facilitate planning studies and to introduce complimentary methods for evaluating adaptive treatments. Synthetic images and contours are generated for each fraction of a typical fractionation schedule using principal component analysis and a novel method of sampling coefficients that preserves temporal trends in the data (e.g. tumor regression). Using the synthetic datasets, a series of adaptive schedules ranging from no adaption to daily replanning are simulated and compared to quantify adaptive benefits and characterize tradeoffs with frequency. Daily replanning resulted in significant reductions in all normal tissue planning metrics when compared to no adaptation, and incremental reductions were observed with each increase in replanning frequency while the magnitude of average reductions decreased with each step. Modest correlation between absolute change in planning target volume over the course of treatment and reductions in both mean lung dose and mean esophageal dose were observed. 


\section{Introduction}

\subsection{Problem statement}

Cancer ranks second, behind heart disease, among the leading causes of death in the United States for both men and women by a margin of less than $2 \%$. In 2009 , the most recent year for which data is available, 567,628 cancer deaths were reported, accounting for $23.3 \%$ of all mortalities.$^{[1]}$ For 2013, The American Cancer Society estimated 580,350 cancer-related deaths and projected 228,190 of those to be associated with malignancies of the lung, making lung cancer the number one cause of cancer-related death in the United States. ${ }^{[2]}$

Treatment depends on type, site, and stage of disease, and may be influenced by patient specific preferences or comorbidities, but generally includes surgical resection, chemotherapy, radiotherapy, or a combination of the three. In the case of lung cancer, radiotherapy is indicated in early stage disease where comorbidities or patient refusal preclude a surgical approach, and in advanced cases where tumor is unresectable, making it the treatment of choice for approximately $40 \%$ of newly diagnosed lung cancer patients. ${ }^{[3]}$

Prognosis for those diagnosed with lung cancer remain poor; in their most recent report, The National Cancer Institute reports a 5 year survival rate of $16.6 \%$ for those with cancers of the lung. ${ }^{[4]}$ Low survival rates for the large portion of patients diagnosed with lung cancer coupled with the fact that $40 \%$ receive radiation therapy as the primary treatment modality make improvements in radiation therapy for lung cancer an important topic of research.

For patients receiving radiation therapy, local tumor control and survival have been shown to improve with increased doses; ${ }^{[5,6]}$ however, for most patients deliverable dose is limited by 
associated normal tissue toxicity. Reducing normal tissue dose for a given treatment regimen is a common theme in radiotherapy research and allows tumor doses to be escalated until an isotoxic effect is reached for the limiting normal structure.

Advancements in the field that have lead to increased sparing of normal tissues include: conformal radiotherapy, intensity modulated radiation therapy (IMRT), and tools that reduce uncertainties associated with treatment and setup (e.g. image guidance). Adaptive radiation therapy (ART), which is the subject of this thesis, utilizes these techniques in a dynamic approach to accommodate interfraction variation and may be utilized to further reduce exposure of relevant risk structures.

A basic introduction to traditional radiation therapy, ART, and relevant advancements in the field are presented in this section as context for the work presented in later chapters.

\subsection{Radiation therapy}

Radiation therapy seeks to eradicate diseased tissue through the targeted administration of ionizing radiation while minimizing effects on surrounding normal tissues. Biological effects of ionizing radiation include disruption of cellular DNA, resulting in mutations that may compromise cellular function or ultimately prove fatal for the cell. Damage may result from direct action on the DNA, or indirectly through the production of free-radicals - principally, highly reactive oxygen species - which may subsequently lyse base pair bonds. Irreparable damage may signal programmed cell death (apoptosis) or may inhibit cellular division (mitotic death) and thereby end the life of the cell.

The type of radiation utilized may be either gamma-rays or particulate radiation (e.g. electrons) of various energies and is administered via a beam that originates outside of the patient (external-beam radiotherapy) or by placing radioactive sources inside or next to the 
treatment site (brachytherapy). For lung cancer, treatment usually involves external-beam radiotherapy that delivers gamma-rays in the megavoltage range.

Common to all modalities of radiation therapy is the specification of robust target volumes to ensure tumor coverage with a high degree of probability. In order to specify robust target volumes, geometric uncertainties inherent in the planning process must be appreciated and accounted for. A description of the uncertainties associated with radiotherapy for lung cancer are given below.

\subsubsection{Uncertainties in radiotherapy for lung cancer}

The various uncertainties associated with specifying and localizing radiotherapy targets have traditionally been managed through the addition of safety margins to account for intra- and inter-fraction variations. Specifically, uncertainties are related to: identifying and delineating tumor volumes as seen on planning CTs, ascertaining the extent of microscopic invasion and nodal involvement, patient positioning, and motion of target and surrounding anatomy. Anatomical change throughout treatment presents another challenge to accurately localizing structures of interest and may include: tumor regression, patient weight variations, and the development or resolution of local pathology (e.g. atelectasis, pleural effusion, etc.).

\subsubsection{Identifying and delineating gross tumor}

After acquisition of a diagnostic CT scan obtained with the patient in the intended treatment position, organs at risk and gross tumor are outlined by a physician using treatment planning software. While tools exist to aid in segmentation, ultimately contours are based on clinical judgment and have been shown to vary significantly for different observers. In one study, inter-observer variability as defined by the standard deviation of observer contour distances from a common region of interest (voxels designated as gross tumor by at least $50 \%$ of observers) was 
$1.02 \mathrm{~cm}$ for lung lesions when based on diagnostic CT alone. This improved to $0.42 \mathrm{~cm}$ in a second phase of the study that incorporated FDG-PET to help distinguish gross tumor; however, variability of this magnitude still constitutes a significant uncertainty in radiotherapy. ${ }^{[7]}$

Discrepancies at this stage of planning represent systematic errors that are propagated throughout treatment and for some patients may be the dominant source of uncertainty. Causes of variability may include lack of knowledge, inconsistencies in methodology, and lack of contrast between gross tumor and surrounding normal structures or pathology (e.g. atelectasis). ${ }^{[8]}$

\subsubsection{Extent of microscopic disease}

By definition, the gross tumor volume delineated by the physician includes macroscopic disease; however, surrounding tissue may harbor cancerous cells that are not clinically detectable. The extent of subclinical disease represents an additional uncertainty in the planning process that is managed by adding a margin around the grossly detectable tumor. Margins are based on histological evaluations of excised tumors and depend on, among other things, the type and grade of cancer. In a study conducted by Giraud et al., microscopic extension from the gross tumor border for lung cancer ranged from 0.0 to $12.0 \mathrm{~mm}$. For patients included in the study, 8 and 6 $\mathrm{mm}$ accounted for $95 \%$ of the observed microscopic disease in adeno- and squamous-cell carcinomas respectively. ${ }^{[9]}$

\subsubsection{Setup}

After regions of interest have been delineated and a plan developed, the patient is positioned for daily treatments with focus on reproducing the planning geometry. A reference point within the patient is designated when the planning CT is first acquired, and marks are made on the patient's skin for positioning in the coordinate system of the LINAC using in-room lasers. Relative motion of the skin and internal anatomy, the precision with which skin marks are made, 
and the care with which marks are aligned, all contribute to setup error which is composed of both systematic and random components. Though errors vary depending on institution, and from patient to patient, an estimation of $2-4 \mathrm{~mm}$ has been reported for lung cancer. ${ }^{[10]}$

\subsubsection{Respiratory motion and management}

The effects of respiration on tumor motion are complex and vary widely from patient to patient in magnitude, period, and trajectory, thus patient-specific analysis and management are recommended. ${ }^{[11]}$ In a study conducted by Stevens et al., tumor motion due to respiration in the superior-inferior direction ranged from $0.0-2.2 \mathrm{~cm},{ }^{[12]}$ thus respiratory motion represents a significant uncertainty in radiation therapy. In the planning process, respiratory motion can introduce image artifact in the simulation CT leading to systematic errors in both delineation and dose calculation. During treatment, inter- and intra-fraction motion may cause further deviations in dose from the planned distribution.

Motion management may include: additional target margins to encompass the range of motion throughout treatment; gating using a surrogate for tumor location (e.g. abdominal surface) to restrict beam-on time to a particular phase of motion; breath-hold using a spirometer and other forms of bio-feedback to isolate the tumor in a given location; forced shallow breathing with abdominal compression to limit movement; or tumor-tracking in which the beam position is dynamically modified. Each method warrants separate analysis and appropriate margins for residual errors.

\subsubsection{Tumor regression}

Regression of the gross tumor volume throughout the course of radiotherapy has been observed by various authors. Kupelian was one of the first to quantify this change in non-smallcell lung cancer (NSCLC) reporting an average volume decrease of $1.2 \%(0.6-2.3 \%)$ per day. ${ }^{[13]}$ 
Britton et al. acquired weekly 4DCT datasets for 8 NSCLC patients receiving definitive radiotherapy and found a median decrease of $41.7 \%(8.1-71.3 \%) .{ }^{[14]}$ Finally, van Zwienen et al. performed a more extensive study consisting of 114 lung cancer patients with weekly cone-beam CTs (CBCT), 46 (40\%) demonstrated tumor regression with an average volume change of $37 \%$ over the course of treatment. ${ }^{[15]}$

\subsubsection{Patho-anatomical change}

The development or resolution of local pathology throughout treatment may dramatically affect the anatomy of a given region and significantly alter planned dose distributions. In lung cancer, two types of patho-anatomical change are of interest. 1) Atelectasis is a collapse of lung parenchyma that may be due to obstruction of a main airway. Affected regions often have similar CT appearance to gross tumor which may make it difficult to distinguish between the two.

2) Pleural effusion is an accumulation of excess fluid in the space between the visceral and parietal pleura that surround the lungs, compressing and displacing adjacent regions.

\subsubsection{Target volumes and planning}

Various volumes are specified in the planning process to effectively target diseased regions. International Commission on Radiation Units (ICRU) report $50^{[16]}$ formalizes target volume definitions and recommends the following volumes be specified: the gross tumor volume (GTV), which consists of grossly detectable disease; the clinical target volume (CTV), which is an appropriate expansion of the GTV to incorporate potential subclinical disease; and the planning target volume (PTV), which is an expansion of the CTV to account for residual errors in setup and motion. While GTV and CTV specify regions of anatomy that presumably harbor disease, the PTV is a region of space that exists in the coordinate system of the linear accelerator 
(LINAC) specified to ensure a high probability of coverage in the presence of geometric uncertainty.

After delineation of target volumes and risk structures on the CT image, planning is carried out using treatment planning software to specify a number of beams and calculate the resulting dose distribution within the patient. Appropriate beam angles help avoid excessive irradiation of risk structures along with collimation to limit the extents of the beam to PTV.

\subsubsection{Conformal radiation therapy}

Conformal therapy is a technique that further modifies the beam to conform to the shape of the PTV as opposed to a rectangular field that is limited to the extents of the target volume. Beam shaping is achieved by inserting a custom cutout in the path of the beam that conforms to the $2 \mathrm{D}$ projection of the target volume as observed through the beams-eye-view or by using a multi-leaf collimator (MLC) which is mounted in the gantry of the linear accelerator. The MLC consists of multiple leaf pairs that are manufactured of a high- $\mathrm{Z}$ material and can be inserted into or retracted from the path of the beam automatically to create custom apertures.

\subsubsection{Intensity modulated radiation therapy and inverse planning}

While beam shaping increases dose conformality, certain distributions may remain unachievable (e.g. concave dose distributions). In intensity modulated radiation therapy (IMRT), non-uniform fluence patterns for a given beam angle and aperture are delivered; practically this is achieved by delivering multiple smaller beam segments (beamlets) to build up a fluence profile. The superposition of multiple non-uniform fluence profiles allows a greater degree of flexibility in achievable iso-dose shapes, and may increase dose gradients and conformality, but 
also increases treatment complexity. The number of plan-design parameters are significantly increased in IMRT making manual parameter selection and design impractical.

Computer assisted optimization of plan parameters is an essential element of modern IMRT. Treatment intent is translated into $n$ mathematical objectives $F_{k}, k=1 \ldots n$, that make up a composite cost function $F(\tau)$, where $\tau$ is a set of planning parameters to be optimized.

$$
F(\tau)=\sum_{k=1}^{n} F_{k}
$$

Objective functions may be based on physical expressions of dose or more complicated biological models of treatment effect. Additionally, a set of constraints $C(\tau)$ may also be specified and the optimization seeks the solution:

$$
\begin{aligned}
& \min _{\tau} F(\tau), \\
& C(\tau) \leq 0
\end{aligned}
$$

In this work, IMRT optimizations utilize dose volume criteria, and objective functions are of the general form:

$$
F_{k}=w_{k} \sum_{i \in V}\left(\frac{d_{i}-d_{k}}{d_{k}}\right)^{2} \Delta v_{i}
$$

Where $w_{k}$ is an importance weighting factor, $V$ is the volume of interest, $d_{i}$ is the current dose for voxel $i, d_{k}$ is the intended dose for voxel $i$, and $\Delta v_{i}$ is the fraction of volume $V$ represented by voxel $i$. Weighting factors are specified by the planner and significantly affect resulting plans.

The optimizer begins with an initial estimate of the discrete intensity map of beamlets, or opening density matrix (ODM), and iteratively seeks a solution. For multi-modal cost functions, 
solutions may converge to sub-optimal local minima depending on initial approximations and the search algorithm employed.

\subsubsection{Image-guidance}

The use of onboard imaging devices is an important advancement in modern radiation therapy. Images acquired on the day of treatment and in the treatment geometry provide up-todate positional information on target and normal tissue structures, allowing deviations from the planning geometry to be detected and corrected for. Increases in positional accuracy justify commensurate reductions in safety margins, which decreases dose to surrounding tissue, and potentially increases the therapeutic ratio of treatment.

The frequency with which image-guidance is utilized is important in determining safety margin reductions. Image-guidance strategies that are implemented periodically throughout treatment must allow for residual interfraction errors; when daily-guidance is utilized, safety margins need not include such considerations.

The effect of using daily image-guidance on safety margins has been reported in the literature by various authors. Yeung et al. commented on the significance of cone-beam CT in fractionated radiotherapy for lung, reporting a reduction of approximately $1.5 \mathrm{~cm}$ in PTV margin when compared to alignment protocols that relied on surface marks alone. ${ }^{[17]}$ In another study, Bissonnette et al. calculated population based margins assuming daily CBCT and remotecontrolled couch adjustments to setup the patient; they concluded that post-correction setup margins should be on the order of 2 to $3 \mathrm{~mm} .^{[18]}$

Safety margins utilized in this work were based on a protocol produced by the Radiation Therapy Oncology Group (RTOG) assuming daily image-guidance, and are consistent with those suggested by Bissonnette. 


\subsubsection{Fractionation}

In addition to conforming dose to target structures, fractionation (i.e. administering multiple fractions of prescribed dose over a period of time) maximizes the therapeutic ratio of treatment by capitalizing on inherent differences in radiosensitivity of clonogenic and nonclonogenic cellular populations. Briefly, tissue specific dynamics related to repair of damage, repopulation of cells, oxygenation of tissues, and assortment into radiosensitive stages of the cellular cycle, dictate differences in irradiated tissue response. Repair of sub-lethal damage in normal tissues exceed that in the tumor, thus fractionation spares normal tissues while still eradicating clonogenic populations. In addition to repair, repopulation of normal-tissue cells in the time between treatments enhances the sparing effect of fractionation. For the tumor, fractionation increases free-radical production as oxygen is restored, and allows redistribution of cells into radiosensitive stages of the cell cycle, both of which increase the effectiveness of the delivered dose.

\subsection{Adaptive radiation therapy}

Traditionally, a single arrangement of beams and apertures based on an initial planning image are delivered throughout the course of treatment. Plans are created to be robust against variation and other uncertainty through the addition of safety margins to the primary target volumes.

Adaptive radiation therapy (ART) was first introduced by Yan et al. ${ }^{[19]}$ and was defined as a feedback loop that incorporates measurements of treatment variation in modifying and reoptimizing the treatment plan, as opposed to a fixed plan approach. The early work of Yan focused on mitigating geometrical uncertainty as a means to escalate dose. ${ }^{[20,21]}$ However, in its most general sense, ART may incorporate any additional measurement in improving the different 
aspects of treatment and delivery with the objective of improving patient outcome and care. Lof et al. proposed an adaptive control algorithm incorporating characterizations of geometry, fluence, and biology obtained throughout treatment into a dynamic optimization of dose..$^{[22]}$ As alluded to by Lof, de la Zerda later suggested that ART fits naturally into a control theory framework where closed-loop control produces actions based on measurements of a given system in order to drive the system to some desired state ${ }^{[23]}$ this is illustrated in Fig. 1.1.

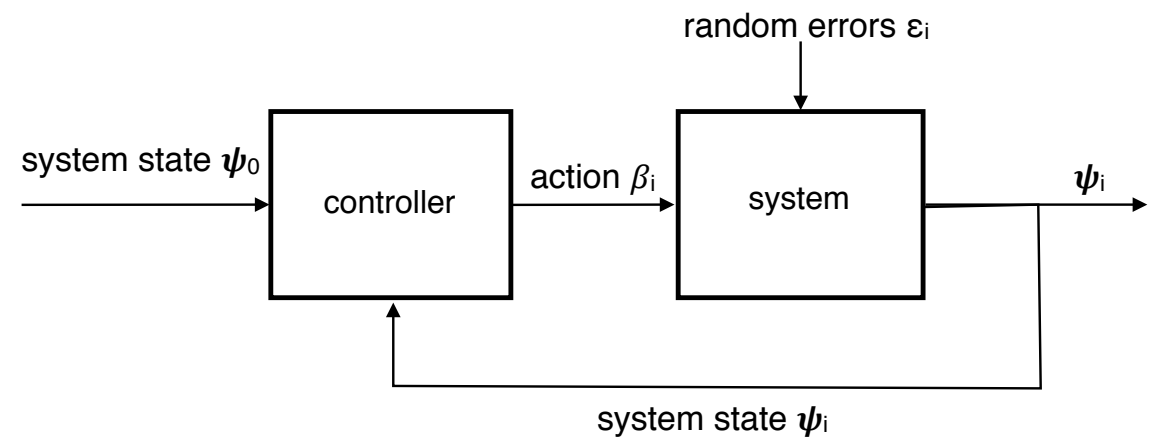

Fig. 1.1 Closed loop control as presented by de la Zerda.

In the context of radiation therapy, additional measurement is facilitated by onboard imaging which allows routine monitoring of patient-specific setup error, motion, treatment response, and provides a means of calculating dose on daily anatomy.

\subsubsection{Margin reduction}

As mentioned, Yan et al. was the first to suggest ART as a method to decrease margins and escalate dose. Specifically, they proposed replacing the standard population margin early on in treatment with a patient-specific model of random and systematic setup error based on an initial set of portal images. They found that an adequate error estimate could be obtained after relatively few measurements, resulting in smaller margins and allowing additional dose for $64 \%$ of patients in their study. ${ }^{[21]}$ Later work by Martinez et al. ${ }^{[24]}$ considered 150 prostate patients revealing an escalative benefit of $5 \%(2.5-10 \%)$ and $7.5 \%(2.5-15 \%)$ for 3D conformal and 
intensity-modulated ART strategies respectively, ultimately concluding that achievable dose escalation is patient dependent.

In 2007 Hugo et al. ${ }^{[25]}$ extended the approach Yan used in prostate and evaluated various strategies that incorporated patient-specific margins for lung. In total, the study compared 5 different approaches including: a population based margin; a hybrid margin that incorporated patient-specific measurement of respiratory motion based on initial fluoroscopy, and population estimates of all other error; an offline adaptive strategy that used an initial set of images to estimate patient-specific inter- and intra-fraction variation; an online margin that performed a daily online correction for mean target position and incorporated a patient-specific measurement of respiratory motion based on initial fluoroscopy; and finally, an online adaptive margin which also performed a daily online correction of the mean target position but utilized an initial set of images to determine patient-specific variation. The study reported a population margin of $9.7 \mathrm{~mm}$ and a reduction to $4.5 \mathrm{~mm}, 2.9 \mathrm{~mm}$, and $2.8 \mathrm{~mm}$ for the offline adaptive, online, and online adaptive strategies respectively. The authors conclude that the online strategies would be useful for patients exhibiting large variability while the offline strategy would be more appropriate for patients with less variability in the daily mean tumor position.

Using four-dimensional cone beam computed tomography, Harsolia et al. ${ }^{[26]}$ performed a similar study comparing 3D-conformal, 4D-union internal target volume (ITV), 4D-offline, and 4D-online adaptive strategies, to account for setup error. Compared with the 3D-conformal plan they reported reductions of $15 \%, 39 \%$ and $44 \%$ in PTV volumes for the above strategies respectively. 


\subsubsection{Re-planning}

In addition to estimations of patient specific setup error, frequent imaging allows monitoring of target volume dynamics (e.g. changes in size and shape) and other anatomical change (e.g. atelectatic resolution). In cases of large anatomical deviations from the planning geometry, re-planning may be necessary to maintain proper coverage and acceptable levels of toxicity. In cases where coverage and toxicity are acceptable, re-planning may have the potential to improve these endpoints.

\subsubsection{Adapting to tumor regression}

Treating regressing volumes with a static margin may result in unnecessary irradiation of normal tissue; a fact that may be exploited by ART. Ramsey et al. ${ }^{[27]}$ suggested a weekly adaptation to accommodate tumor regression and evaluated whether a dosimetric advantage existed for such a strategy. The advantage of weekly ART over a non-adaptive plan was quantified by the reduction in the volume of ipsilateral lung receiving at least $20 \mathrm{~Gy}$ (V20) for both a geometric test case and a retrospective simulation carried out in 7 patients. For the geometric test case, various spherical targets with initial volumes ranging from $25-1000 \mathrm{cc}$ were utilized, and simulated ART yielded a mean reduction in V20 of $21.2 \%(17-24 \%)$. For the patient simulations, adaptive plans and dose calculations were performed on the planning kilo-voltage CT ( $\mathrm{kVCT}$ ) in conjunction with adaptive PTVs derived from weekly mega-voltage CT (MVCT) scans. Using this approach, a mean reduction in V20 of 17\% (5-20\%) was reported.

Woodford et al ${ }^{[28]}$ examined the effect of various adaptive strategies on mean lung dose (MLD) and V20 for a subset of 3 patients in a study primarily concerned with quantifying tumor regression. Adaptive plans were based on reduced-field-of-view MVCTs merged with an initial 
$\mathrm{kVCT}$ and used rigid registration for image alignment. For a single patient exhibiting a pattern of early regression and a total decrease in tumor volume of $87 \%$, adaptive plans corresponding to fractions 5, 15, and 22 were produced. Adapting at these three time points for this patient reduced MLD and V20 by 15 and 13\% respectively. A single adaptation corresponding to fraction 16 was simulated for another patient, decreasing V20 by 7.4\% and MLD by $4.1 \%$. For the remaining patient, re-planning was associated with fraction 21, reducing V20 and MLD by $3.2 \%$ and $2.4 \%$ respectively. While the population and analysis was limited, a trend of reduced lung-dose with increased adaptive-frequency is evident; furthermore, the pattern of regression and timing of adaptation are stated as important in determining derived benefits. The authors recommend a threshold of $30 \%$ observed-regression occurring within the first 22 fractions as a "useful criterion" for determining which patients might benefit from ART. However, patient selection using the criterion could only be applied after the $22^{\text {nd }}$ fraction.

The most recent study investigating tumor regression and ART was carried out by Guckenberger et al. ${ }^{[29]}$ and included 13 patients with NSCLC receiving conventionally fractionated 3D conformal radiation therapy. Weekly helical CTs were used for re-planning which was performed during the third and fifth weeks of treatment. Dose accumulation was carried out using a surface-based deformable image registration (DIR) algorithm and was projected onto the planning dataset. Average MLD for all patients was reduced from $17.7 \pm 3.9$ Gy to $17.0 \pm 3.9 \mathrm{~Gy}, 16.9 \pm 3.9 \mathrm{~Gy}$, and $16.5 \pm 3.8 \mathrm{~Gy}$ using a single adaptation in week 3 , a single adaptation in week 5 , and an adaptation in both weeks 3 and 5 respectively. The twice adaptive strategy allowed dose escalation from $66.8 \pm 0.8 \mathrm{~Gy}$ to $73.6 \pm 3.8 \mathrm{~Gy}$ using an isotoxic MLD criteria. 
While gross tumor is readily distinguished by volumetric imaging, uncertainty remains regarding the presence and location of microscopic extension. Hugo et al ${ }^{[30]}$ used deformable image registration to propagate planning contours to weekly helical CT image-sets for examining the effect of online GTV-based alignment on CTV coverage. The study concluded that large errors in CTV localization may result in the context of significant or anisotropic tumor regression.

In a follow up study, Guckenberger et al ${ }^{[31]}$ examined the effect of ART on CTV dosing for two extremes of CTV dynamics i.e. stationary microscopic disease (MD) and MD that shrinks commensurate with gross tumor regression. For coverage at $50 \mathrm{~Gy}$ they found that their adaptive strategy achieved adequate dosing of the CTV for both simulated scenarios. Coverage at $60 \mathrm{~Gy}$ was reduced from $92 \% \pm 10 \%$ to $73 \% \pm 19 \%$ for the stationary case, but was restored when applying an isotoxic boost. Thus the authors conclude that ART as implemented in this study does not under-dose the microscopic extension.

The studies mentioned here demonstrate an advantage for an adaptive strategy that accommodates tumor regression, and a general trend of increased benefit with additional adaptations can be observed.

\subsubsection{Online and offline strategies}

Re-planning consists of the same steps involved in the initial planning process including: image acquisition, segmentation, optimization of beams and apertures, dose calculation, quality assurance, and plan approval. To date, only offline approaches, in which re-planning is carried out in the time between treatment fractions, have been implemented clinically. Online strategies, in which image acquisition, planning, and treatment all occur while the patient is on the couch, have been suggested but are currently limited by various practical and technological 
considerations. Such an approach will presumably improve treatment by reducing setup error and improving consistency between planning and treatment geometries.

\subsubsection{Workload considerations}

Presumably adaptive benefit will increase with increasing frequencies of adaptation; however, frequent adaptation is currently costly requiring the same steps involved in the initial plan listed above. Implementing daily replanning represents a large increase in associated workload. It is important, therefore to characterize the tradeoff between adaptive frequency and resulting benefit to inform decisions regarding allocation of resources. Methods to predict which patients may benefit from adaptive therapy are likewise desirable.

\subsection{Purpose and thesis overview}

The purpose of this dissertation is to assess the potential benefit of ART for lung cancer and to present tools to accommodate its implementation and evaluation. While various studies have examined the benefits associated with a limited number of re-plans, this work examines the potential of ART in the limit of daily re-planning and analyzes the tradeoff between re-planning frequency and dosimetric advantage. Furthermore, ART represents a shift in treatment and planning paradigms, thus evaluation tools that reflect this change are warranted.

\subsubsection{Overview of specific aims}

At the onset of this work the following specific aims were proposed in conjunction with the purpose stated above: 1) construct a software tool to facilitate implementation of adaptive radiation therapy in an existing treatment planning system;2) generate a set of synthetic images and contours corresponding to each fraction of treatment for a cohort of lung cancer patients using principal component analysis (PCA); 3) quantify the benefit associate with daily adaption 
for lung cancer and characterize the tradeoff between replanning frequency and adaptive benefit; and 4) investigate methods for identifying patients that may benefit from an adaptive therapy approach. An overview of these aims is given below.

\subsubsection{Aim 1: Adaptive therapy software tool}

Develop a software tool as a plugin to an existing treatment planning system to facilitate implementation of adaptive radiation therapy and serve as a platform for conducting simulations of adaptive therapy treatments. The tool will extend existing functionality in the temporal domain providing methods for organizing and managing large sets of planning objects inherent in adaptive treatments that incorporate multiple images and plans. Methods for displaying and navigating temporal sequences of relevant planning metrics will also be included.

\subsubsection{Aim 2: Synthetic patient datasets}

Generate a set of synthetic images, contours, and mappings corresponding to each fraction of a 35 fraction treatment schedule for a cohort of lung cancer patients. Datasets will be generated using PCA analysis and should exhibit regression trends of the primary tumor that are representative of those observed clinically. Generated datasets will be utilized in simulations of various adaptive therapy schedules.

\subsubsection{Aim 3: Potential benefit of ART as a function of re-planning frequency}

Utilizing synthetic imagesets generated in the previous aim, conduct a series of planning studies with the objective of quantifying adaptive benefit associated with daily replanning and characterizing the tradeoff between adaptive frequency and resulting benefit. Simulations will be carried out for different frequencies of adaptation from no replanning up to daily replanning. Benefit will be quantified in terms of dose sparing and allowable escalation. 


\subsubsection{Aim 4: Identifying patients that benefit from adaptation}

Using the results from the previous aim explore potential methods for identifying patients that may benefit from an adaptive therapy approach.

\subsubsection{Overview of thesis}

This document is based on additional works included here as appendices which serve as the primary documentation for the outlined specific aims. Remaining chapters seek to summarize and supplement these documents while presenting the information in a cohesive form. Chapters 3 and 4 correspond to manuscripts intended for publication contained in appendices II and IV respectively; the reader is advised to first review the relevant appendix before proceeding with these chapters. 


\section{Treatment history browser}

Tools to facilitate adaptive radiotherapy planning on the scale implemented in this work were not readily available at the time of its writing and an initial effort was dedicated to this purpose. Incorporation of additional data (images and contours) associated with time-points throughout treatment for the purpose of replanning represents a change in the current treatment paradigm. Though the dynamic nature of treatment is appreciated and accounted for through the addition of treatment margins, planning and evaluation methods are inherently static i.e. they are based on a single instance of patient anatomy acquired prior to radiation delivery. Workflows and data-objects reflect this static paradigm and are ill-equipped in an adaptive approach that carries out replanning at multiple time-points.

Essential extensions of an effective treatment planning system (TPS) for ART include: 1) temporal planning objects that reflect the four-dimensional nature of treatment, 2) methods for efficiently managing and navigating large amounts of treatment data, and 3) evaluation methods that accommodate multiple datasets corresponding to different time-points. As part of this work, a software tool that incorporates these elements is developed as a plugin to the Pinnacle treatment planning system (Philips Oncology, Fitchburg, WI) using the Python programming language and Qt GUI toolkit; the tool is referred to here as the "Treatment History Browser"

(THB). A discussion of treatment planning system (TPS) elements that may facilitate adaptive workflows and their implementation in this work are given in the sections below. Detailed information on installation, usage, and class-documentation for the THB can be found in the Users Guide located in Appendix I. 


\subsection{Temporal planning objects}

In traditional radiation therapy a CT scan is acquired before treatment, and regions of interest (ROI), including target and risk structures, are delineated and approved by a physician. The CT scan and ROIs are used to develop a plan that consists of an arrangement of beams and apertures which are delivered throughout treatment. In the Pinnacle TPS, "trial” objects represent a proposed treatment plan based on a given CT image and consist of beam objects and the associated dose volume; additionally, ROI objects correspond to image volumes and may be associated with any trial that is based on the image.

In an adaptive paradigm, trial objects are created for each image incorporated into the planning process each of which may be used to represent single or multiple treatment fractions. In the limit of daily imaging, a trial object is created for each fraction of treatment and provides an estimation of dose as calculated on the anatomy of the day regardless of whether re-planning is performed. While trials may be used to represent treatment fractions, fraction specific data is not explicitly recorded by the system; to this end a "fraction" object is introduced in the software tool and is created for each fraction of treatment. Each object is associated with a single trial, and naturally includes additional data such as fraction number, date, and whether or not replanning is carried out for the fraction. Temporally-ordered collections of fraction objects make up a given treatment course and are represented by a "treatment" object. Analogous to trials, multiple treatments may exist for each patient and represent proposed treatment options in the evolving adaptive-plan.

Like the images they are associated with, individual ROI objects correspond to single instances of dynamic anatomy. In an adaptive plan, multiple ROI instances associated with the same structure at different time-points exist and necessitate a means of organization and 
management; to this end, the "ROI group" object is created. Though intended for ROIs corresponding to the same structure, ROI groups may consist of any arbitrary set of ROIs which facilitates analysis of sub-regions or those otherwise related. Newly introduced objects and their relation to existing Pinnacle objects are illustrated in Fig. 2.1.

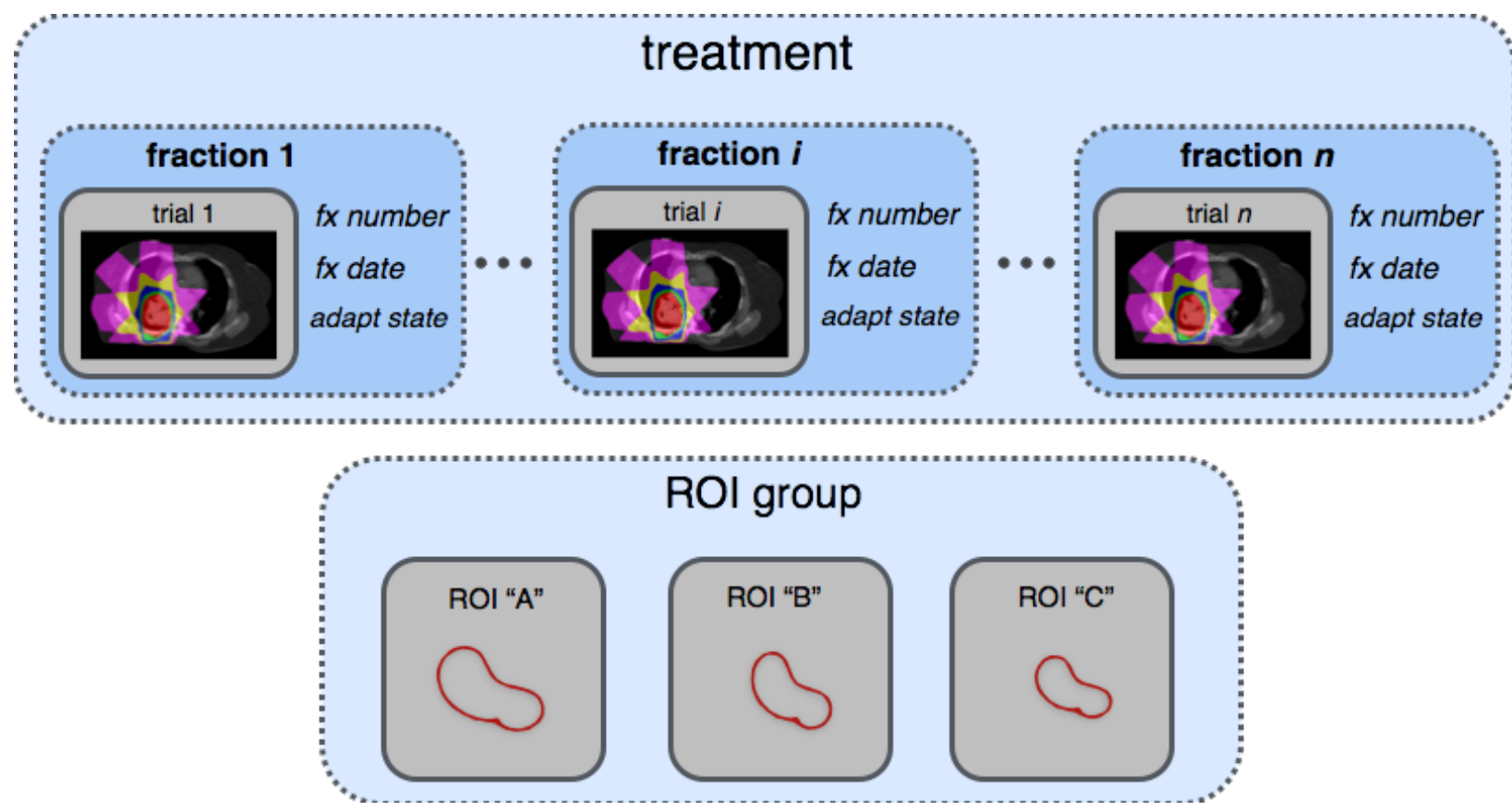

Fig. 2.1 Organizational diagram of THB (dotted border with blue fill) and Pinnacle (solid border with gray fill) software objects. Treatment objects (top) consist of a collection of $\boldsymbol{n}$ temporally ordered fraction objects. Fraction objects are associated with a Pinnacle trial object and include fields for fraction number, date, and adapt state (i.e. whether replanning is carried out). ROI group objects (bottom) are a collection of related Pinnacle ROI objects.

\subsection{Managing large amounts of treatment data}

Implementation of temporal planning objects as described above provides an efficient scheme for managing the large amounts of treatment data inherent in an adaptive treatment. Manipulating individual instances as opposed to group objects (e.g. individual ROIs of an ROI group) is cumbersome and inefficient. Furthermore, group management of objects associated with different time-points builds in a level of standardization in the display of individual instances (e.g. ROI color, image window levels, etc.) and in the evaluation of treatment data (e.g. 
plot line-styles, etc.). This type of consistency lends to greater clarity in treatment related tasks and facilitates the planning workflow.

\subsection{Methods for evaluating treatments that consist of multiple datasets}

\subsubsection{Cumulative dose}

In an adaptive treatment, re-plans are based on recent images of patient anatomy which reflect any variation that has occurred since previous acquisitions and which modify the projected dose distribution of the initial plan. One method for synthesizing data from multiple time-points is to sum individual dose distributions to a single reference image ${ }^{[32]}$ using a mapping established by deformable image registration. This method of evaluation has various advantages: 1) cumulative dose is directly comparable to the original plan which likewise corresponds to a single image, 2) dose-response data is largely based on projected cumulative metrics ${ }^{[33]}$ and 3) ambiguities related to comparing volume-based parameters (e.g. V20) in the presence of volumetric variation $^{[34]}$ are avoided if a common reference is utilized for both.

Dose accumulation utilizes mappings between imaging data to warp dose distributions to a given reference image. Inaccuracies in image registrations will likewise affect deformed distributions, thus reliable dose accumulation depends on accurate mappings between image data. In this work consistency between images and mappings is achieved by generating synthetic displacement vector fields (DVF) using a PCA model and then warping the primary dataset to create images resulting in self-consistent images, contours, and mappings that correspond to intermediate time points (see chapter 3).

In cases where DVFs accurately map dose, any time-point may serve as a valid reference for dose accumulation assuming that mass is conserved between images. In cases where mass is 
not conserved, different images represent distinct structures as opposed to distinct poses of anatomy which presents a subtlety in reference image selection. The following discussion illustrates this point.

\subsubsection{Cumulative dose in the presence of mass loss}

Consider an image $I_{a}$ that consists of a single voxel $V_{a}$ acquired at time-point $t_{a}$ and that receives an initial fraction of radiation. Assuming mass loss occurs as a result of the delivered radiation, the cellular population represented in $V_{a}$ may be divided into a group that survives and persists, denoted by $S$, and a group that is killed or otherwise cleared from the voxel, denoted by $K$. After the initial irradiation, a second image $I_{b}$ consisting of a single voxel $V_{b}$, and containing the surviving fraction, is acquired at time-point $t_{b}$, and an additional fraction of radiation is delivered to population $S$. The scenario is illustrated in Fig. 2.2.

Dose delivered to the cellular population represented in $V_{a}$ at time $t_{a}$ considering separate populations of surviving and non-surviving cells is given by:

$$
D_{V_{a}}=\frac{E_{s}\left(t_{a}\right)+E_{k}\left(t_{a}\right)}{m_{s}+m_{k}}
$$

where $E_{s}$ and $E_{k}$ are the absorbed energy at a given time point for surviving and non-surviving fractions respectively, and $m_{s}$ and $m_{k}$ are the masses of the respective population. Dose delivered to voxel $V_{b}$ at time $t_{b}$ is as follows:

$$
D_{V_{b}}=\frac{E_{s}\left(t_{b}\right)}{m_{s}}
$$

Cumulative dose using image $I_{a}$ as reference is then given by:

$$
C D_{I_{a}}=\frac{E_{s}\left(t_{a}\right)+E_{k}\left(t_{a}\right)+E_{s}\left(t_{b}\right)}{m_{s}+m_{k}}=D_{V_{a}}+\frac{E_{s}\left(t_{b}\right)}{m_{s}+m_{k}}
$$

and incorporating equation (2.2 this becomes: 


$$
C D_{I_{a}}=D_{V_{a}}+D_{V_{b}} \frac{m_{s}}{m_{s}+m_{k}}=D_{V_{a}}+D_{V_{b}} \frac{m_{V_{b}}}{m_{V_{a}}}
$$

Cumulative dose using $I_{b}$ as reference is given by:

$$
C D_{I_{b}}=\frac{E_{s}\left(t_{a}\right)+E_{s}\left(t_{b}\right)}{m_{s}}
$$

and assuming that

$$
D_{V_{a}}=D_{S}\left(t_{a}\right)=D_{K}\left(t_{a}\right)
$$

equation (2.5 may be written as

$$
C D_{I_{b}}=D_{V_{a}}+D_{V_{b}}
$$

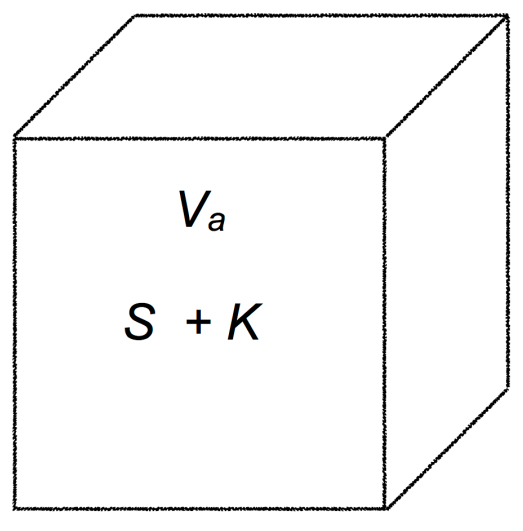

$l_{a}, t=t_{a}$
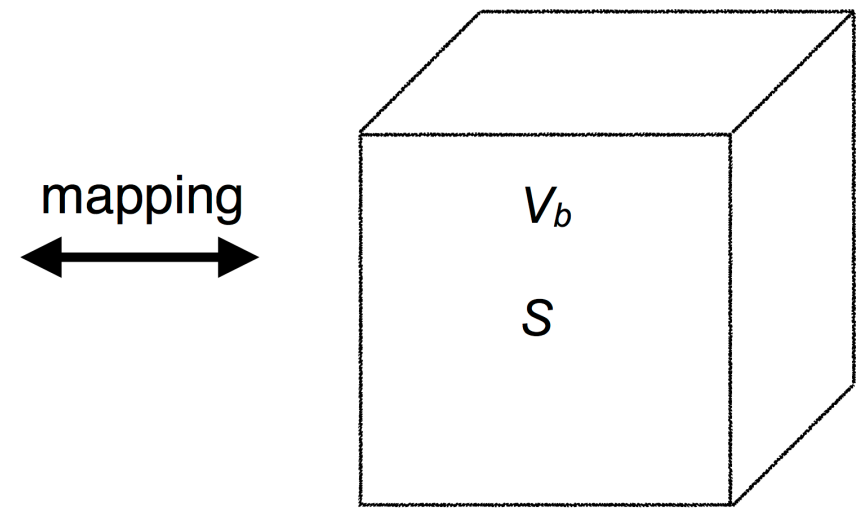

$I_{b}, t=t_{b}$

Fig. 2.2. Dose accumulation in the presence of mass loss. Voxels $V_{a}$ and $V_{b}$ are irradiated at times $t_{a}$ and $t_{b}$ respectively. The surviving fraction of cells is denoted as $S$ and the fraction that does not survive is denoted as $K$. Dose accumulation is facilitated by a mapping between the two images $I_{a}$ and $I_{b}$ either of which may be selected as the reference image.

Equation(2.4 is consistent with a literal interpretation of cumulative dose, i.e. cumulative dose is equal to the actual mean dose received by the cellular population contained in the voxel. In this case, cells that receive less dose because they are eradicated and cleared from the voxel decrease the reported cumulative dose. One may argue, that summing dose in this way may lead 
to an erroneous conclusion that coverage was compromised throughout treatment when in reality coverage was maintained and mass-loss occurred. An alternative then is to report the mean dose that would be received if all cells were to persist throughout treatment. In this case cumulative dose reflects the dose received by surviving cells in each fraction as opposed to the actual dose received by all cells represented in the earlier image. Using the latter definition, cumulative dose reported on the earlier image is equivalent to that reporter on the later image.

These subtleties highlight the limitations inherent in using a static image to summarize dose delivered to dynamic structures that experience mass loss which result from the compression of temporal data to a single time-point. Such considerations motivate development of history-based methods to compliment assessments based on cumulative dose.

\subsubsection{History-based assessment methods}

As highlighted in the previous section, cumulative dose represents a compression of data which obscures the temporal details associated with treatment delivery, i.e. the dose distributions associated with each fraction are compressed to a single time-point.

Temporal sequences of dose metrics, plotted as a function of treatment fraction, are presented here as a complimentary method to accumulating dose for evaluating and informing adaptive plans. It is hypothesized that preserving the temporal course of metrics throughout treatment will provide insight into the dynamics of adaptive benefit i.e. Patterns and trends in temporal signals (e.g. changes in volume, mass, tumor centroid, etc.) may indicate advantageous times to adapt or serve to classify patients that benefit from an adaptive planning approach.

Greater clarity and accuracy in evaluation methods are necessary to relate details of treatment delivery to clinical outcome ${ }^{[35]}$ likewise, it is anticipated that temporal tracking of relevant metrics will provide valuable perspective on treatment delivery and outcome and may 
allow patient specific estimates of response through a correlation of various temporal signals (e.g. mass and cumulative dose), though correlative analysis of delivery metrics and response are not addressed in this thesis.

\subsection{Browser overview}

The main form of the software tool is pictured in Fig. 2.3. The most prominent feature of the graphical user interface is a pair of independent plot areas used for display of signal data. Each plot corresponds to a single signal at any given time but may include data from multiple treatments and ROI groups. Dual plot areas allow for flexible comparisons between different treatments, ROI groups, or signal types and each plot may be opened in an external editor for modifying and saving to disk.

Signals for ROI groups as a function of treatment fraction include: volume, mass, centroid position, min dose, max dose, mean dose, and arbitrary dose-volume-histogram (DVH) based metrics (e.g. V20). Additional signals include: single fraction DVHs, all fraction DVHs, and cumulative DVHs.

Multiple fractions, treatments, ROIs, and signals, contribute to a large amount of data that may be cumbersome to navigate and display in a meaningful fashion. To facilitate this, information is conceptualized as a multi-dimensional data space that may be browsed interactively by the user.

Treatments, ROI groups, and signals are navigated using a group of combo boxes and arrow keys that reside below the plot areas. Fractions are selected using the "Treatment Schedule" calendar which highlights dates associated with each fraction. If a single-fraction signal is selected in the plot area, a change in fraction updates the plot to reflect the new selection; for 
multi-fraction data, the plot is marked with a dashed line or corresponding data is made bold to distinguish the current fraction selection.

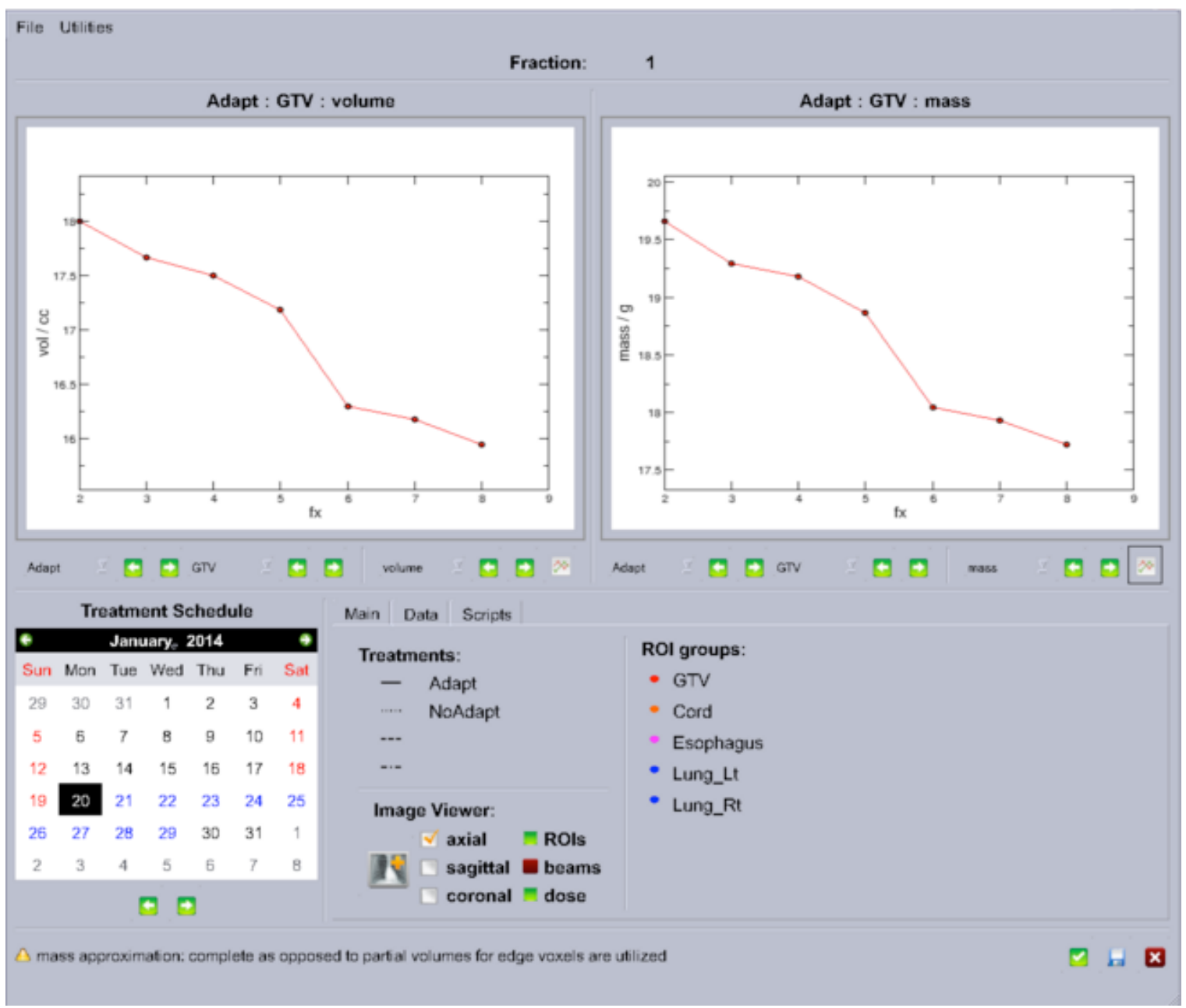

Fig. 2.3 Main form of the "Treatment history browser" software plugin for the Pinnacle TPS

Below the plot area and adjacent to the treatment calendar are three tabs: the "Main" tab, the "Data" tab, and the "Scripts" tab. The "Main" tab indicates line-styles assigned to treatments, and colors associated with ROI groups, both of which are held constant in all plots. On the same tab are a group of controls for an image viewer that reflects the current fraction selection which is included for convenience and may consist of axial, sagittal, and coronal views. The "Data" tab gives a summary of image and plan names associated with a given treatment indicating which fractions are adaptive, and is used to initiate automatic replanning as implemented in the latter 
portion of this work. Lastly, a "Scripts" tab allows quick navigation of the file system to initiate relevant scripts.

In addition to the main window, forms for managing ROI groups and treatment details (Fig. 2.4) may be called from the "utilities" menu at the top of the main window; for detailed information on usage the reader is again referred to Appendix I.

Upon exiting the tool, all data pertaining to the active session is saved to disk in a single file. This allows previous sessions to be restored even if underlying data no longer persists in the TPS. While active, signal data is stored in a python dictionary object and is updated only when retrieving new data or when forced by the user. This model was implemented to increase responsiveness of data retrieval and decrease the memory burden associated with a large amount of treatment data i.e. image and planning data can be discarded after relevant metrics have been extracted.

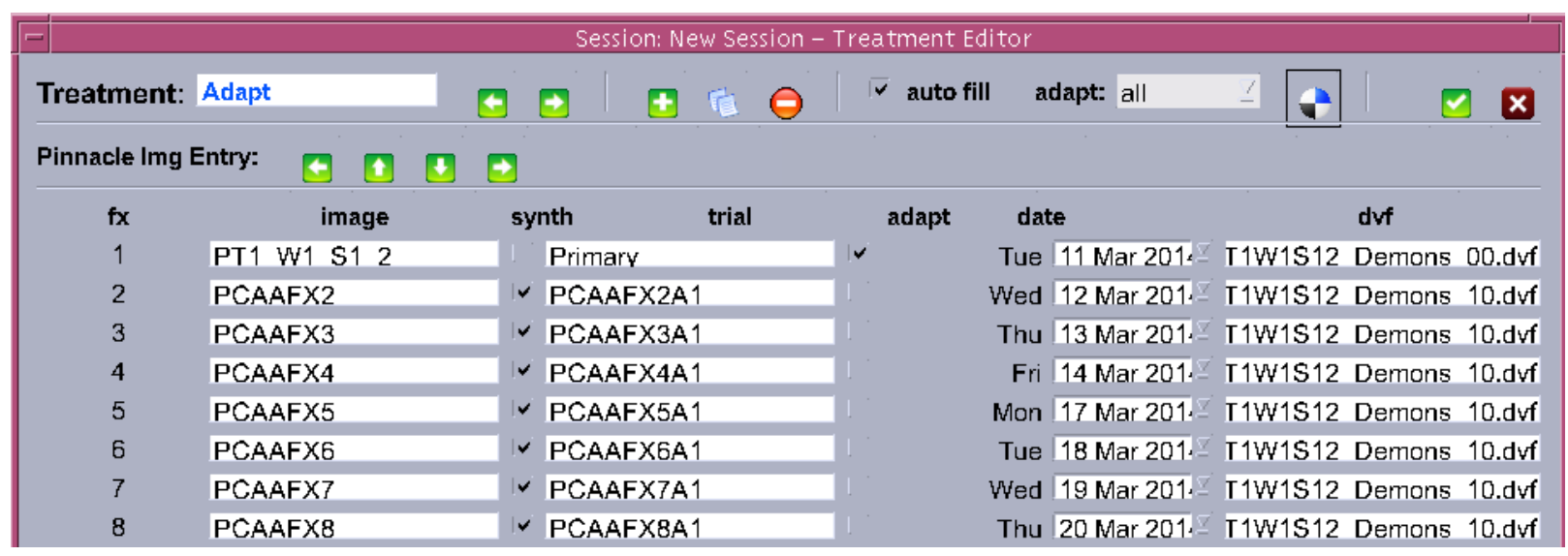




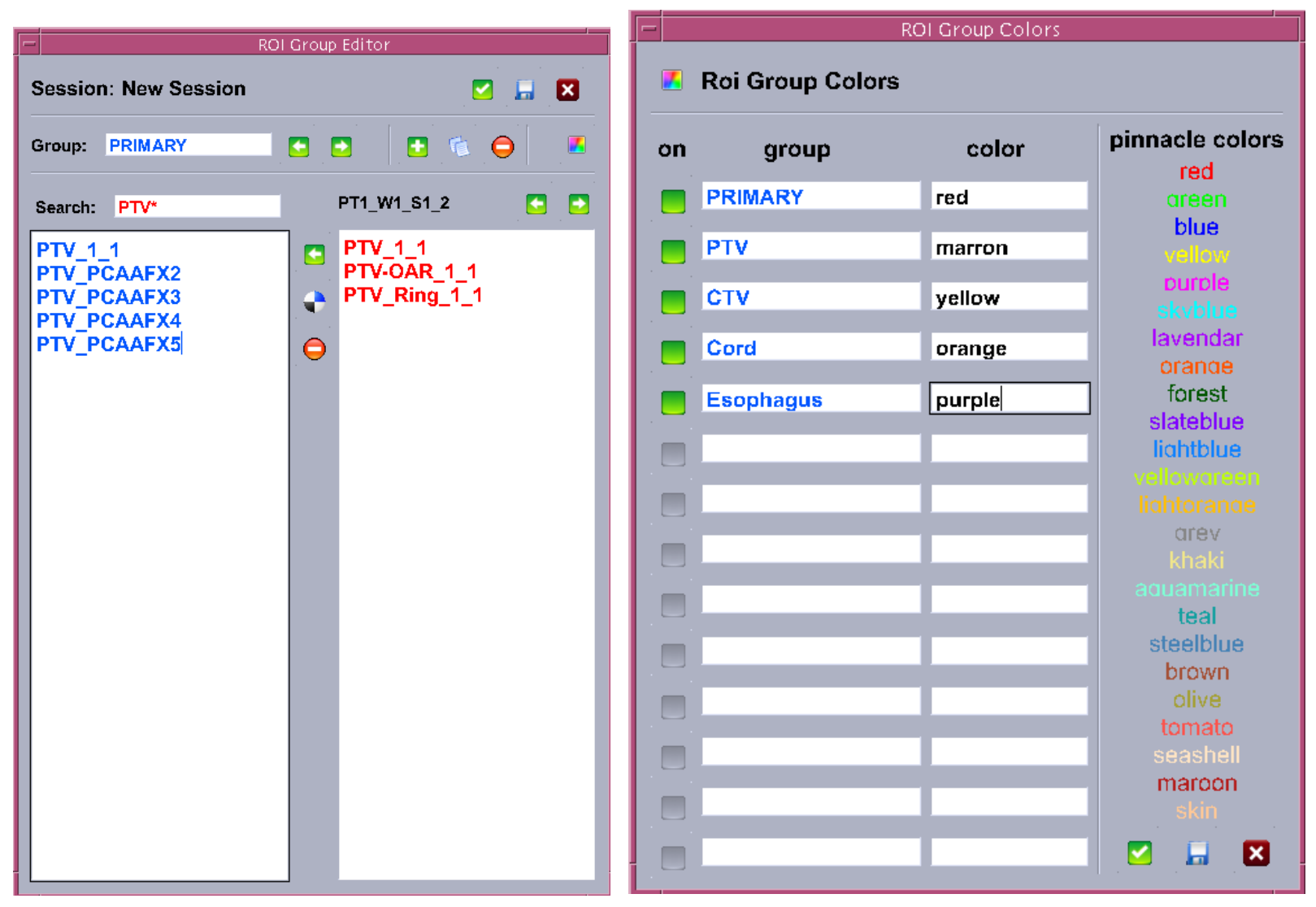

Fig. 2.4 Treatment editor, ROI group manager, and ROI color forms. 


\section{Synthetic patient datasets}

CT datasets of patient anatomy in the treatment position currently serve two main purposes in radiation therapy planning: 1) datasets provide a geometric model of the patient that is used to localize risk and target structures, and arrange beams in a manner consistent with treatment objectives; and 2) CT datasets contain electron density information that is utilized in estimating the dose to target and risk structures associated with intended beams.

In this chapter, planning studies investigate the dosimetric benefit associated with adapting the initial plan to accommodate interfraction variation. This is carried out at different frequencies up to modifying each fraction and thus requires an image and set of contours for each day of the prescribed treatment regimen. Daily images and contours for the patient cohort included in this thesis were not readily available and thus synthetic datasets based on existing data were generated.

\subsection{Statistical modeling of inter-fraction variation using principal}

\section{component analysis}

A statistical model of interfraction variation was utilized to generate synthetic datasets corresponding to each fraction of a 35 fraction treatment regimen. The model is derived from a temporal sequence of images and a set of mappings, which identify corresponding tissue locations in either a pair or group of images, using PCA. The method is briefly described here; for a more detailed treatment, the reader is referred to Appendix II.

For each set of patient images, a mapping is established between the first image in the sequence and all subsequent images using deformable image registration as illustrated in Fig. 3.1. 


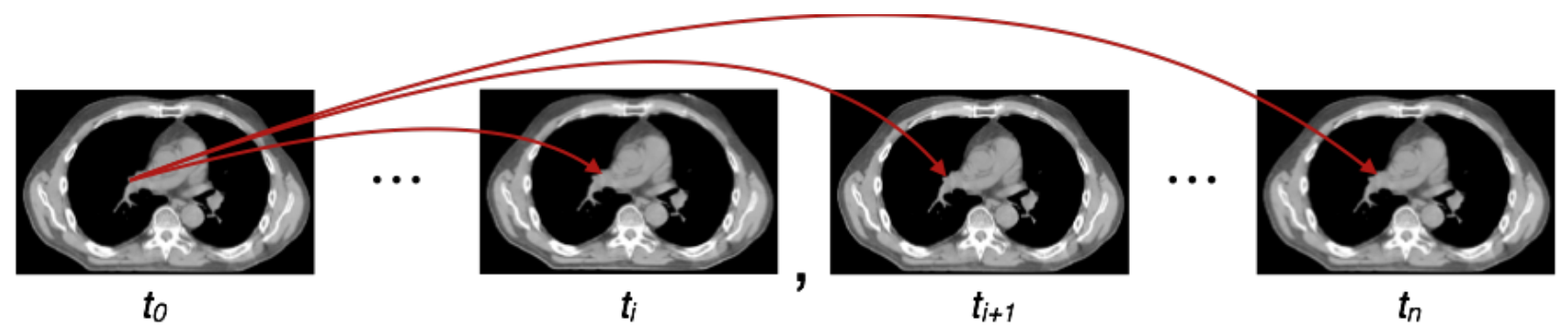

Fig. 3.1 Registration of a temporal series of $n$ images. Registrations are performed between first image in series and all subsequent images to identify corresponding tissue locations in the group of images. (mapping for single point illustrated).

A common region of interest is used for each patient, resulting in DVFs of equal dimensions that track displacement of initial-image tissue elements throughout the course of treatment. Each

DVF is then reshaped into column vectors which are combined to make up a joint variation matrix containing homologous elements along each row and which proceed temporally from left to right. The matrix is mean-corrected by subtracting row means from each row and a factorization of the matrix is performed using PCA. This results in a set of basis vectors and weighting coefficients which may be utilized to reconstruct the original data; i.e. a weighted sum of basis vectors utilizing coefficients associated with a given transform produces the same transform. Reasonable DVFs that are not observed in the original data may likewise be constructed using a summation of basis vectors by selecting appropriate weighting coefficients.

Relevant coefficients are drawn from the model by sampling from a statistical distribution in a way that preserves temporal trends in the original data. This is accomplished by first plotting coefficients corresponding to all DVFs for a single basis vector against the elapsed time associated with each coefficient, and performing a linear fit of the data. Fit residuals are then used to construct a vector-specific probability density function (PDF) using kernel density estimation from which samples may be drawn. Synthetic coefficients are generated by evaluating the fit at time-points associated with each treatment fraction and adding a sampled component from the residual distribution. 
Fraction images and contours are then produced by deforming the first dataset in the series using corresponding transforms resulting in a set of images, contours, and mappings that are self consistent. Inconsistent datasets, in which discrepancies exist between mappings and the actual position of tissue elements and contour boundaries, may lead to inaccuracies in deformed dose distributions and derived metrics which may lead to faulty conclusions regarding treatment delivery. Therefore, consistent datasets are important in this work for two reasons: 1) cumulative measures of dose, which depend on underlying mappings, are utilized as a means of evaluating planning studies; and 2) ambiguities in dose coverage of the clinical target volume (CTV) associated with adapting to regressing target volumes are avoided if the initial CTV can be accurately tracked throughout treatment.

\subsection{Patient input data}

Statistical modeling to generate synthetic datasets is based on weekly images acquired throughout the course of radiation therapy treatment for 12 patients diagnosed with non-smallcell lung cancer. For each patient, 4 - 6 weekly helical CT scans are acquired under an active breathing control (ABC) protocol and relevant contours (gross tumor volume, lungs, cord, esophagus, heart and nodal involvement) are delineated on each scan by a qualified physician. Patients exhibited variation in size, location, and stage of disease as summarized in Table 3.1, and percent gross tumor volumes as a function of treatment week are illustrated in Error! Reference source not found.. The latter is of particular interest in this thesis as planning studies implementing adaptive re-plans presume benefit commensurate with tumor regression.

Prior to deformable registration, a rigid alignment of weekly images is performed to align boney anatomy, and all images are resampled on to a grid associated with the first image in the series. 
PATIENT CHARACTERISTICS

\begin{tabular}{|c|c|c|c|c|c|c|c|c|c|}
\hline patient & stage & location & nodes & $\begin{array}{l}\text { tumor vol } \\
\text { (cc) }\end{array}$ & patient & stage & location & nodes & $\begin{array}{l}\text { tumor vol } \\
\text { (cc) }\end{array}$ \\
\hline 1 & IIIA & LUL & Y & 24 & 9 & IIIA & RUL & $\mathrm{Y}$ & 40 \\
\hline 3 & IIIB & Bilateral & $\mathrm{Y}$ & 100 & 14 & IIIA & RML & $\mathrm{Y}$ & 34 \\
\hline 4 & IIB & LLL & $\mathrm{N}$ & 65 & 17 & IIIA & RUL & $\mathrm{Y}$ & 216 \\
\hline 5 & IIIB & RUL & $\mathrm{N}$ & 1 & 18 & IIIB & RUL & $\mathrm{N}$ & 58 \\
\hline 6 & IIIA & RLL & $\mathrm{N}$ & 242 & 20 & NA & LLL & $\mathrm{Y}$ & 47 \\
\hline 8 & IIB & RUL & $\mathrm{N}$ & 11 & 21 & NA & LUL & $\mathrm{N}$ & 86 \\
\hline
\end{tabular}

Table 3.1. Patient characteristics for the 12 NSCLC patients utilized in the work. $L U L=$ left upper lobe; $L L L=$ left lower lobe; $\mathrm{RUL}=$ right upper lobe; $\mathrm{RML}=$ right middle lobe; $\mathrm{RLL}=$ right lower lobe.

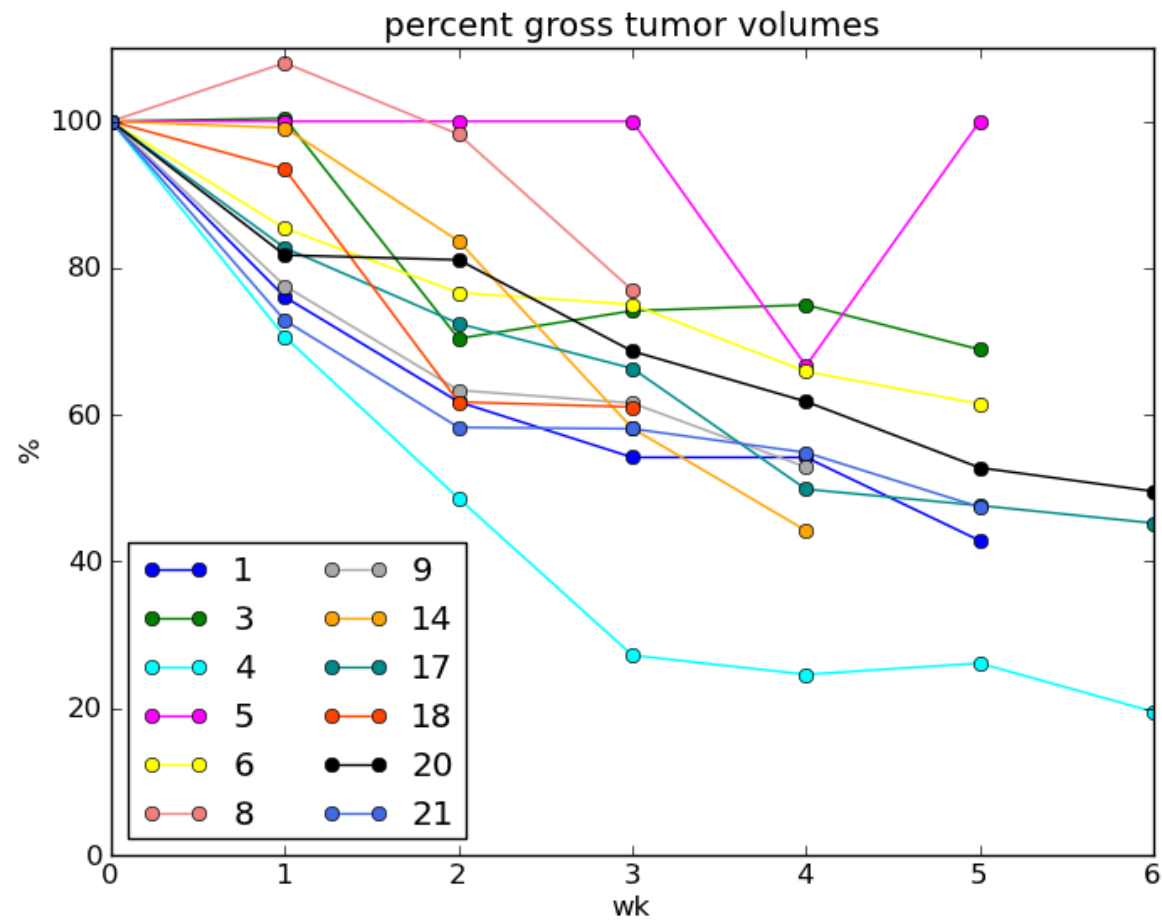

Fig. 3.2 Percent gross tumor volumes as a function of treatment week based on physician delineated contours.

\subsubsection{Deformable registration of weekly images}

Ultimately, statistical models of inter-fraction variation reflect mappings between images used in the analysis as opposed to observable variation in the images themselves. Though DVF 
accuracy is not a primary objective of this work, potential DIR algorithms were evaluated on their ability to map the extents of visible tumor and preserve observed regression trends.

Two deformable registration algorithms for use in generating synthetic datasets are considered in this work: 1) the Demons algorithm as implemented in the Pinnacle treatment planning system (Philips Oncology, Fitchburg, WI), and 2) a small deformation, inverse consistent, linear elastic (SICLE) algorithm. ${ }^{[36]}$ Evaluation consisted of propagating GTV contours from the first image to the last in the series using each algorithm and comparing them to the manually-drawn physician contours on the final image.

Discrepancies between propagated and physician-drawn contours for a subset of the patient cohort are reported in Table 3.2 and were smallest for the Demons algorithm ( $p=0.02$ paired ttest ). Based on this analysis, the Demons algorithm is utilized in registrations between existing weekly images for statistical modeling of inter-fraction variation and resulted in $n-1$ DVFs for each patient with $n$ weekly images.

In summary, patient data utilized in statistical modeling of interfraction variation consisted of $3-5$ DVFs, the first image in the series, and a set of contours corresponding to the first image for 12 NSCLC patients. 
PROPAGATED AND MANUAL GROSS TUMOR VOLUMES (CC)

\begin{tabular}{ccccc}
\multicolumn{5}{c}{ PROPAGATED AND MANUAL GROSS TUMOR VOLUMES (CC) } \\
\hline \hline patient & $\begin{array}{c}\text { manual } \\
\text { (first image) }\end{array}$ & $\begin{array}{c}\text { manual } \\
\text { (final image) }\end{array}$ & $\begin{array}{c}\text { SICLE } \\
\text { (final image) }\end{array}$ & $\begin{array}{c}\text { Demons } \\
\text { (final image) }\end{array}$ \\
\hline 1 & 26 & 10 & $22(+12)$ & $16(+6)$ \\
3 & 100 & 70 & $82(+12)$ & $63(-7)$ \\
4 & 66 & 13 & $44(+31)$ & $40(+27)$ \\
6 & 250 & 127 & $180(+53)$ & $133(+6)$ \\
8 & 12 & 9 & $11(+2)$ & $5(-4)$ \\
17 & 207 & 98 & $132(+34)$ & $105(+7)$ \\
18 & 96 & 36 & $109(+74)$ & $33(-3)$ \\
20 & 49 & 23 & $38(+15)$ & $38(+15)$ \\
21 & 110 & 50 & $83(+33)$ & $57(+7)$ \\
\hline \hline
\end{tabular}

Table 3.2. Gross tumor volumes corresponding to the first and last weekly images for a subset of the patient cohort. For the final image, gross tumor volumes are reported for the manually drawn physician contour, the propagated contour using the SICLE algorithm, and the propagated contour using the Demons algorithm. Volume discrepancies between the propagated and manual contours on the final image are included in parentheses. Demons algorithm demonstrated smaller discrepancies between propagated and manual volumes on the final image $(p=0.02)$.

\subsection{Synthetic data}

Synthetic data generated from the 12 patients are reviewed here and consisted of a set of images, contours, and mappings for each fraction of treatment. A selection of synthetic axial image slices corresponding to the centroid location of the GTV for the first, middle, and last fractions are presented in Fig. 3.3 and Fig. 3.4. Regression of gross tumor is evident in the figures, and tumor volumes as a function of treatment fraction are reported in Fig. 3.5. Regression trends are linear for most patients and are devoid of large acute variation due to the sampling method employed in the modeling (see Appendix II). Trends are consistent with those observed clinically, ${ }^{[28]}$ and absence of acute variation is a desirable characteristic for the planning studies in this work.

Artifacts were observed for a large portion of patients in the cranial and caudal extents of synthetic images as a result of registration uncertainties in these regions. An example of artifacts associated with patient 1 are illustrated in Fig. 3.6. Though artifacts of this nature are undesirable, all fell outside dose-grid regions constructed to contain the whole of the lungs and 
other relevant risk structures, thus the impact on planning studies is likely to be negligible.

1

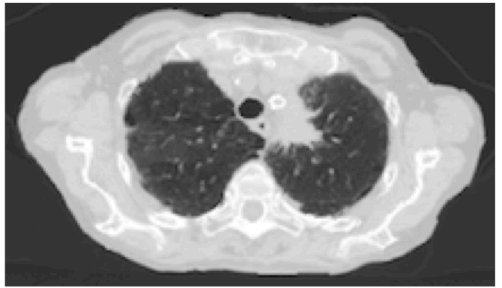

3

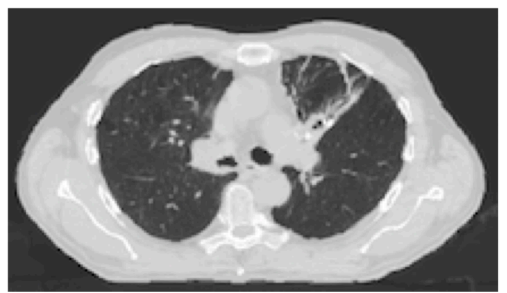

4

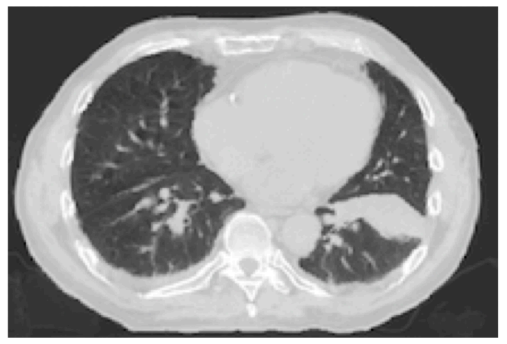

5
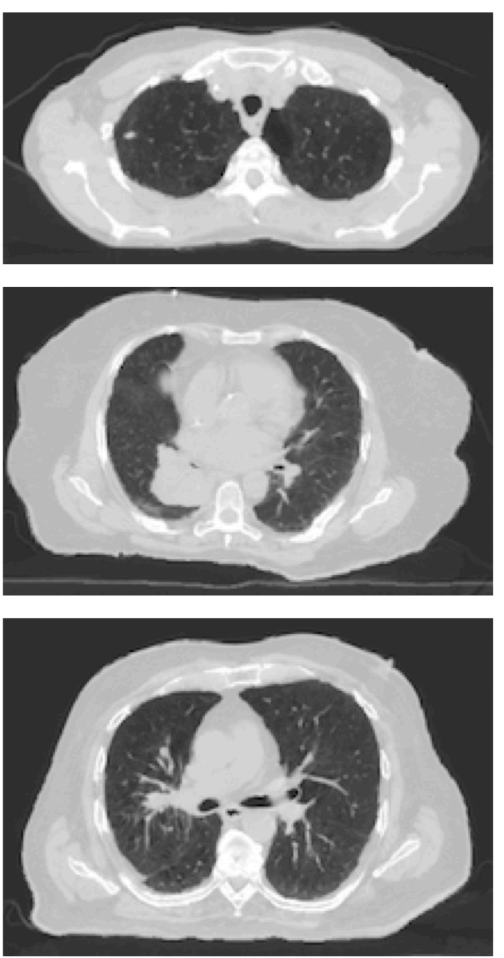
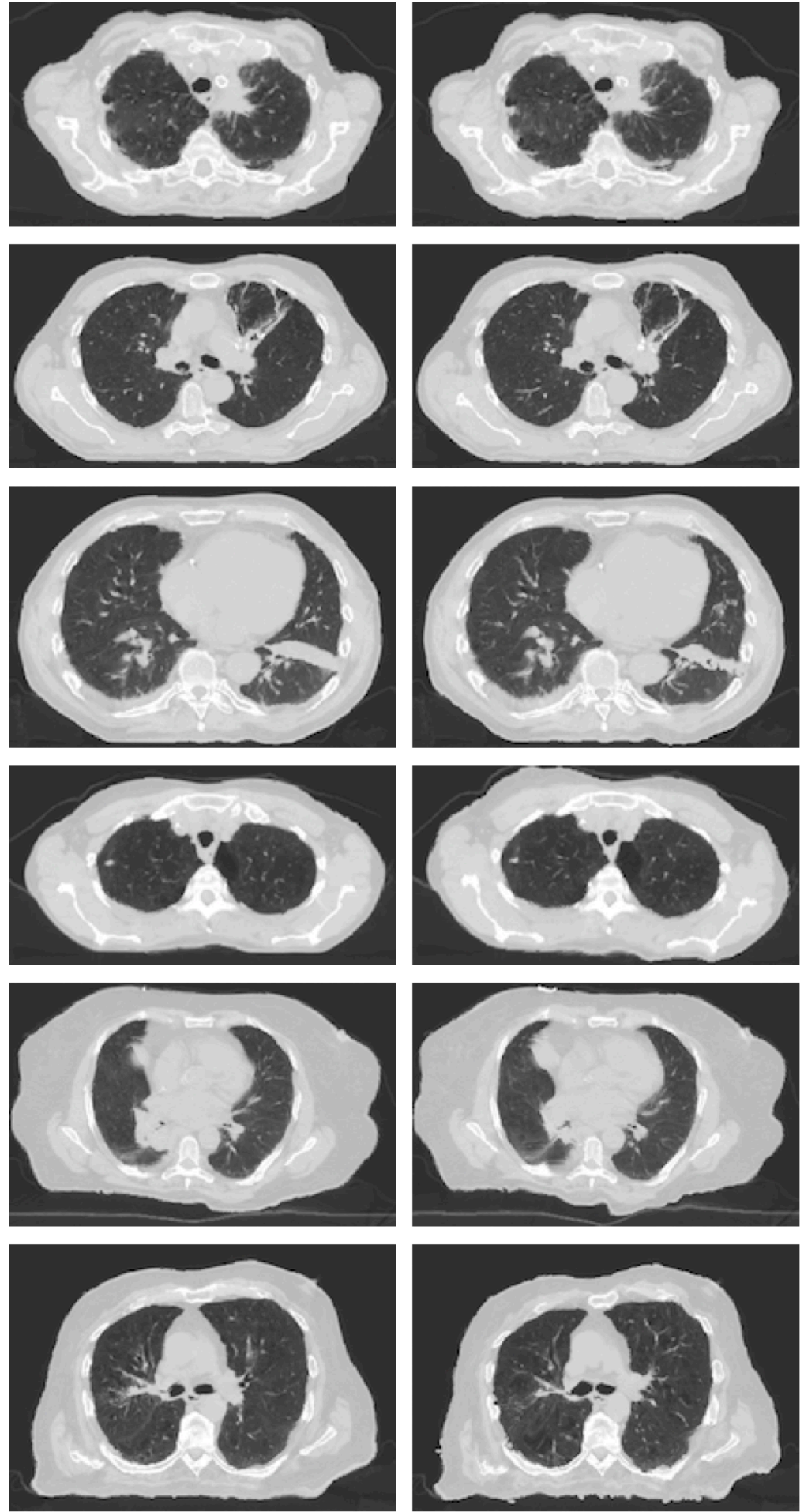

Fig. 3.3 Synthetic images for patients $1,3,4,5,6$, and 8 . Axial slices corresponding to tumor centroid are presented for fractions 1,18 , and 35 (from left to right). 

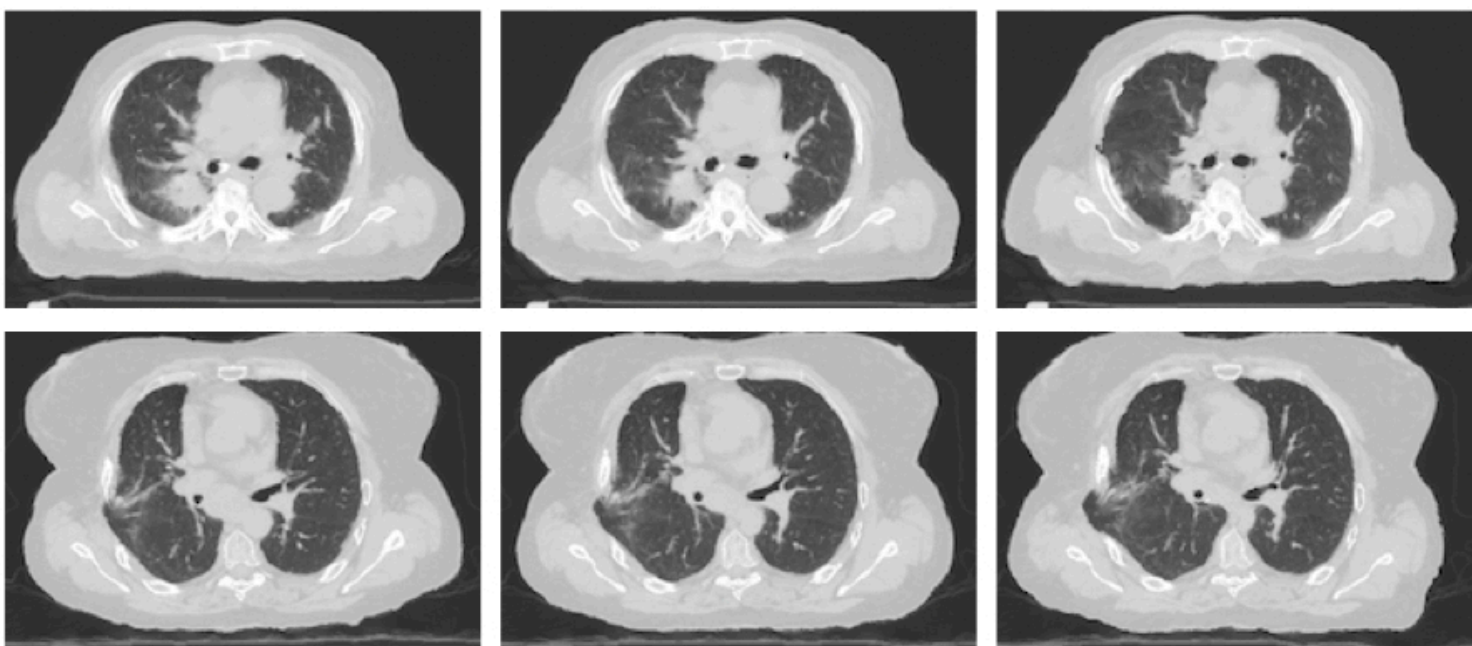

17
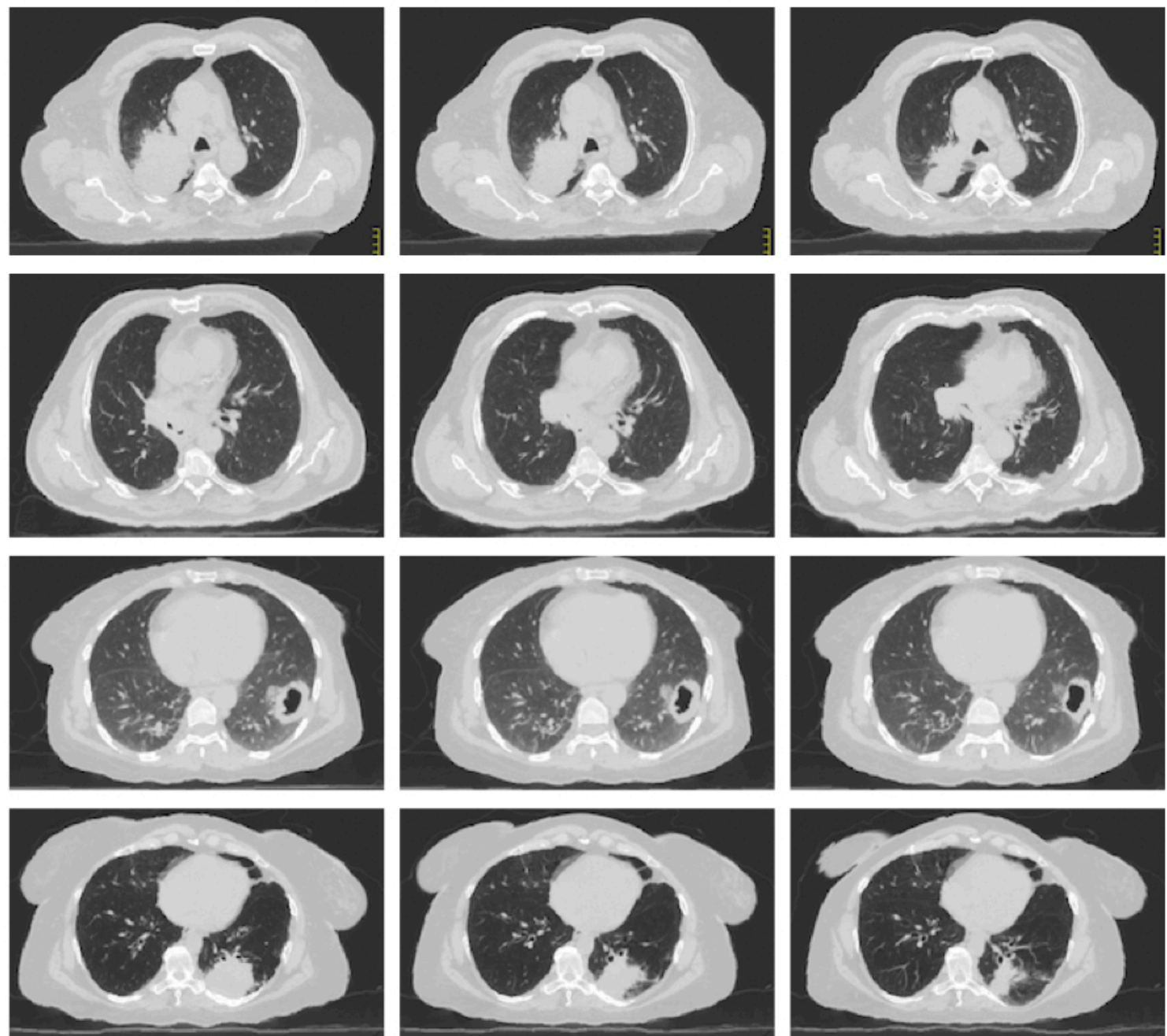

Fig. 3.4 Synthetic images for patients $9,14,17,18,20$, and 21 . Axial slices corresponding to tumor centroid are presented for fractions 1, 18, and 35 (from left to right). 


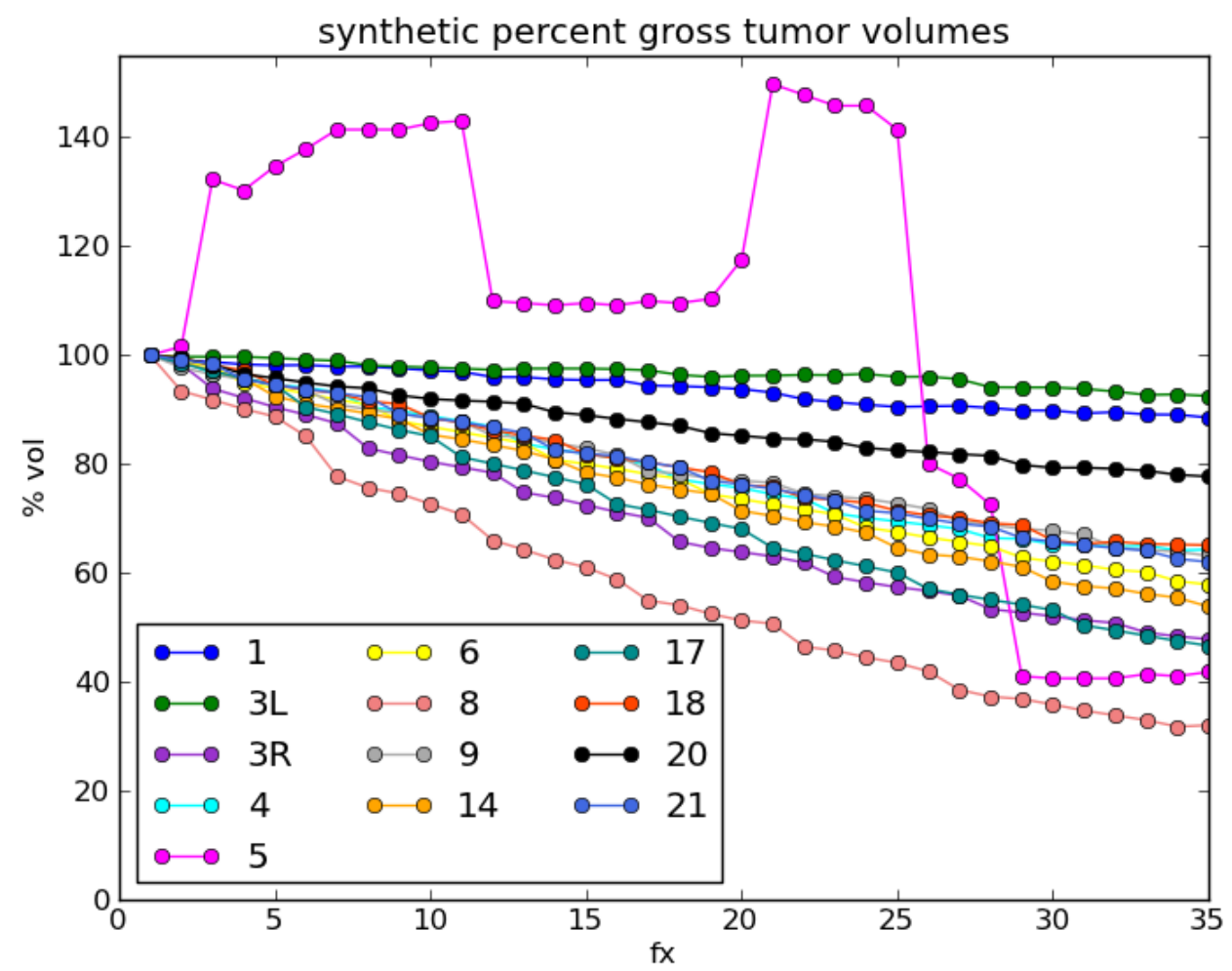

Fig. 3.5 Percent gross tumor volumes for propagated synthetic datasets as a function of treatment fraction.
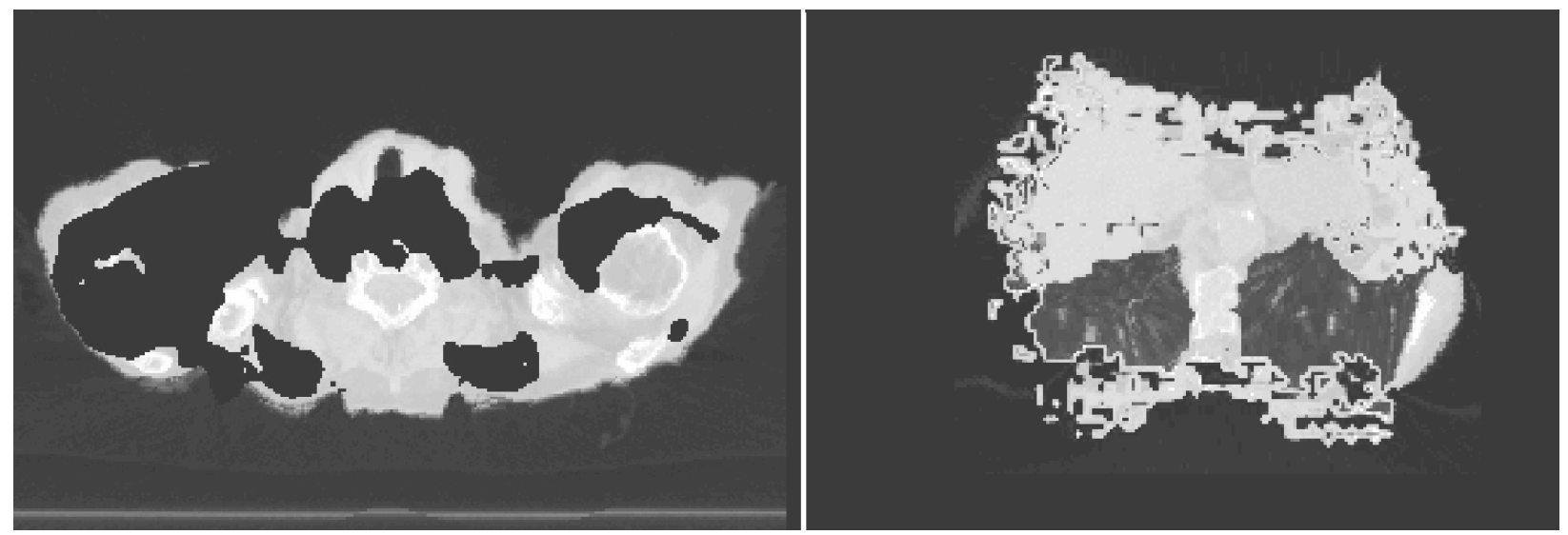

Fig. 3.6 Cranial (left) and caudal (right) artifacts in fraction 35 synthetic image for patient 1 . 


\subsubsection{Synthetic patient cohort}

Though data was available for 12 patients, additional treatment volumes were derived from existing contours to simulate additional patients. This is accomplished by either including or excluding nodal involvement and by separating bilateral tumors into separate volumes. For the twelve patients in this study, 6 had nodal involvement and a single patient had both nodal involvement and bilateral tumors. Dividing the dataset in this way resulted in a total of 20 "patients" available for simulation which are referenced by the original patient number and the addition of an "N," "L," or "R" to denote nodes, left, and right respectively (e.g. 3LN to denote patient 3 that is based on the left tumor and includes nodes). To ensure that study bias is not introduced through this procedure correlation of related patient results is analyzed (see section 4.1).

\subsubsection{Patient target volumes}

For the 20 derived patients, volume measurements of the gross, clinical, and planning target-volume contours are reported in Appendix IV. Individual plots for each are presented for the sake of clarity and to facilitate planning-study analysis of adaptive-benefit as it relates to volume regression. Though related, GTV, CTV, and PTV do not necessarily follow the same regression trends and such subtleties are important to the analysis.

\subsubsection{Data validation}

In order to be suitable for use in planning studies, the following criteria associated with generated datasets were specified: 1) individual synthetic data should represent reasonable poses

of patient anatomy; 2) regression trends should be consistent with those observed clinically; and 3) the set of synthetic patient data should be representative of the range of variation observed in 
practice. These criteria are evaluated in Appendix II and the reader is referred to the same for an explanation of evaluation methods and results. In this section, additional results are presented that are deemed useful in evaluating synthetic datasets.

\subsubsection{Synthetic mean surface distance distributions}

Composite distributions of minimum surface distances (MSD) are described in Appendix II and are utilized to assess similarity in the type and magnitude of variation observed in a sequences of images for a given structure. Comparisons are made between synthetic and actual datasets to verify that the former represent reasonable poses of patient anatomy assuming that global variation can adequately be characterized by a set of contour surfaces and that similarity in variation suggests reasonability of synthetic data. MSD distribution means are utilized in Appendix II to compare synthetic and actual data, and histograms of MSD distributions for each patient and structure are given here to supplement the aforementioned validation.

Agreement was good for the majority of contours and patients, with the largest discrepancies observed in contours that resided in low contrast regions where inter-observer variation in physician-drawn contours and registration uncertainties are likely. 


\section{Adaptive therapy study}

Utilizing the synthetic datasets described in the previous chapter, a study was performed with the purpose of quantifying adaptive benefit resulting from daily replanning, and characterizing the relationship between achievable benefit and replanning frequency. The reader is directed to the study manuscript included in Appendix III before proceeding with the remainder of this chapter which includes supplemental results and discussion.

\subsection{Correlation of nodal and non-nodal treatment results derived from the same patient}

Additional sets of target contours were derived from an original set of 12 patients by separating bilateral tumors and by including and excluding nodal volumes in planning targets as described in 3.3.1. This provided additional types of planning scenarios to be analyzed and allows direct comparison of paired node and non-node plans; however, one concern is that plans based on such datasets will be highly correlated and will therefore introduce bias into study results.

Correlation of reported results for the full-adapt treatment are illustrated in Fig. 4.1.

Though the number of data points are sparse, no strong correlations are observed. Based on this analysis normal statistical methods are utilized to analyze the data. 

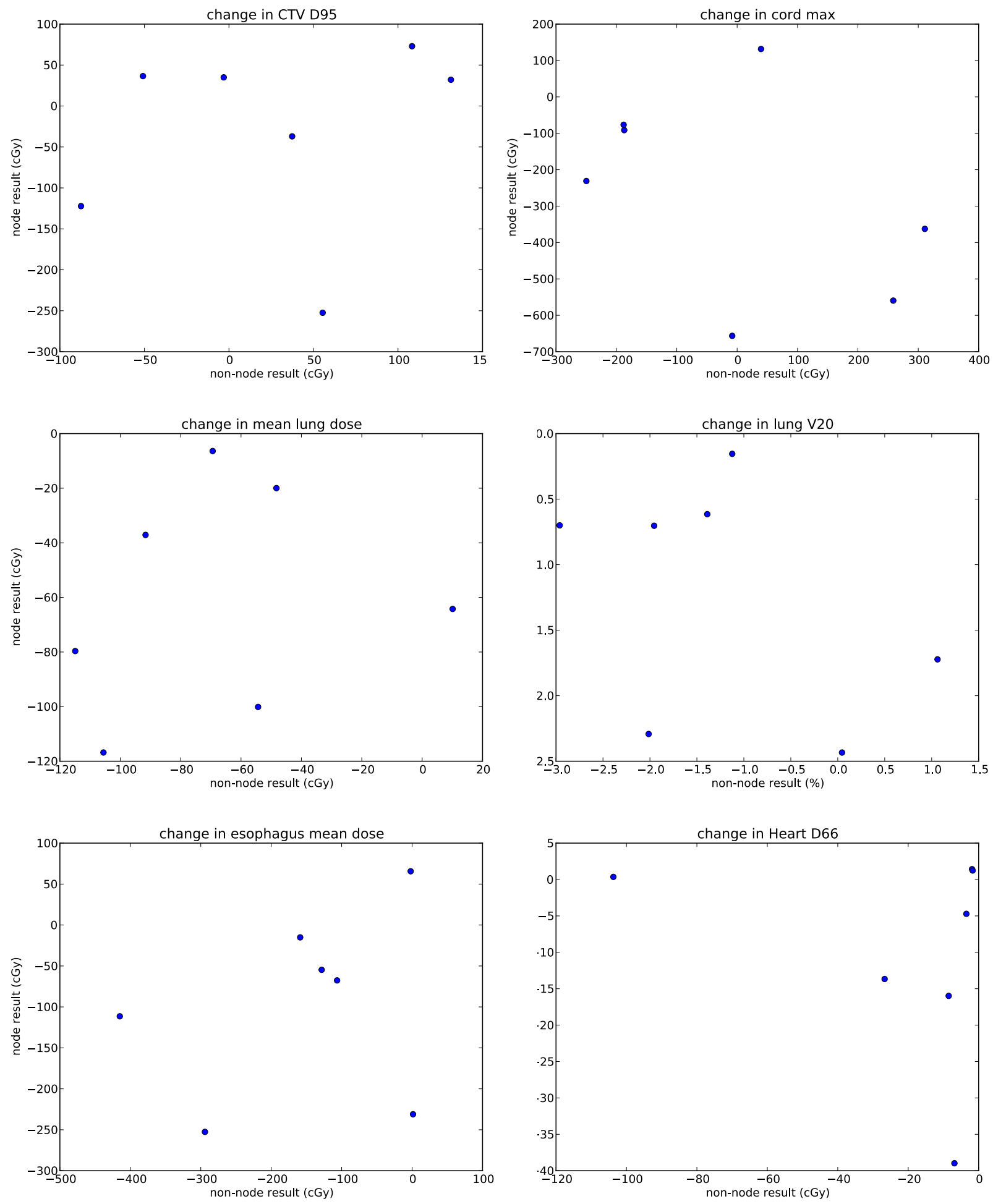

Fig. 4.1 Correlation of dose-metric-difference for non-node and nodal simulations derived from the same patient. Plots are devoid of any strong correlation. 


\subsection{Supplemental results}

Dose volume histograms for CTV and all risk structures are included for all patients and simulations in Appendix VI.

\subsection{Identifying patients that benefit from adaptation}

Three patients in the studies exhibited no benefit from daily adaptation in terms of dose reductions to risk structures of interest including PT5, PT18, and PT20. An additional three patients are identified as receiving a marginal benefit, defined here as a decrease in mean lung dose of less than $50 \mathrm{cGy}$, and included patients $3 \mathrm{LN}, 1 \mathrm{~N}$, and patient $14 \mathrm{~N}$.

As part of the work conducted in Appendix III, various classifications were investigated as indicators of adaptive benefit including nodal status, initial PTV volume, and absolute reductions in PTV volume. Of the three, only the latter proved to be useful in predicting adaptive benefit being modestly correlated with decreases in mean lung dose and mean esophageal dose.

Two of the three patients receiving no benefit (PT5, PT20) from adaptation, and a single patient classified as receiving a marginal benefit (PT1N), exhibited increases in PTV over the course of treatment; however, the remaining patients (PT18, PT3LN, PT14N) all exhibited average decreases in absolute PTV.

It was hypothesized that patterns of regression along with absolute volume changes may be important in predicting adaptive benefit e.g. large acute-variation toward the end of treatment is expected to differ from small daily variation in terms of observable benefit (Appendix IV provides a useful illustration of target volume changes over the course of treatment for all patients considered here). Large variation in patterns of regression was not evident in synthetic datasets potentially due to a degree of imposed linearity in the sampling procedure as discussed in Appendix II. This represents a limitation in detecting the effects of regression patterns in this 
thesis however some variation was observed. PT18 exhibited a unique pattern of volume change over the course of treatment showing a consistent decrease in PTV volume until about fraction 22 at which point a modest incremental increase was observed throughout the remainder of the simulation. Gross tumor regression for the same patient leveled off in the lattermost portion of treatment but did not demonstrate an increase similar to the PTV. While a larger sample size will be necessary to identify relevant predictors of adaptive benefit, PT18 suggests that patterns may indeed be important; furthermore, it highlights the potential for differences in volume change between target volumes for the same patient.

Inter-fraction differences between GTV and CTV variation may produce errors when localizing CTV targets as described by Hugo et. al. ${ }^{[30]}$ These types of discrepancies were apparent for 4 patients in this study (PT1, PT1N, PT5, PT20N) for which CTV increased while gross tumor regressed. In practice, accurate CTV volumes may not exist and adaptations may be implemented based on regression observed in detectable disease. In this case it becomes increasingly important to gauge potential effects as underdosing of the CTV may occur. This is discussed by Guckenberger et. al. who demonstrated that underdosing is mediated in scenarios where dose is escalated to the primary tumor. ${ }^{[31]}$ In a study conducted by Weiss et. al, ${ }^{[37]} \mathrm{CTV}$ contours were propagated to images associated with replanning time-points using deformable image registration. Propagated contours were then used to develop adaptive plans and a simultaneous integrated boost to the primary tumor volume was implemented. In this work, daily CTVs were readily available for all synthetic datasets providing a direct method for producing daily PTVs and avoiding ambiguities; however, this potentially represents smaller reductions in volume than adapting to gross tumor alone. Effectively, reported dose escalation in 
this work boosts dose to the CTV as opposed to primary tumor which may be one factor in the smaller amounts of sparing and associated escalation that were observed.

\subsection{Differences between planning and simulated-treatment dose}

\section{distributions}

Traditional radiotherapy planning utilizes a single image acquired before treatment begins for planning purposes. Dose estimations based on the planning image are expected to vary throughout treatment as plans are delivered to dynamic anatomy that change throughout the course of radiotherapy. Accumulating calculations of dose on daily anatomy to a reference image as implemented in Appendix III provides an improved picture of delivered dose and is utilized as a point of comparison for all adaptive simulations. Though not a primary objective of this thesis, comparisons between planned and accumulated distributions are included to emphasize the importance of using accurate estimations of delivered dose in evaluating adaptive treatments.

Mean differences between the planned dose and cumulative no-adapt simulation for target dose and all normal-tissue metrics are listed in Table 3.1. In general, average differences between planning and cumulative distributions were small; however a large amount of variation was observed for the majority. Differences in mean esophageal dose were statistically significant, while all others were not. For all metrics, differences similar in magnitude to those reported in the adaptive studies were observed. Thus, using the planned distribution as opposed to the cumulative dose of the no-adapt simulation as a point of comparison had the potential to significantly alter patient results. 
MEAN DIFFERNECES BETWEEN PLANNED AND SIMULATED DOSE-DISTRIBUTIONS

\begin{tabular}{ccccccccc}
\hline \hline & planned & & no-adapt & & $\Delta$ & & $\sigma$ & $\mathrm{p}$ \\
\hline CTV D95 & 6942 & cGy & 6958 & cGy & 16 & cGy & 99 & 0.3 \\
cord max & 3814 & cGy & 3754 & cGy & -60 & cGy & 349 & 0.7 \\
mean lung dose & 1346 & cGy & 1363 & cGy & 17 & cGy & 89 & 0.25 \\
lung V20 & 23.9 & $\%$ & 24.1 & $\%$ & 0.3 & $\%$ & 1.9 & 0.1 \\
mean esophagus dose & 2015 & cGy & 2107 & cGy & 92 & cGy & 122 & 0.003 \\
heart D66 & 514 & cGy & 504 & cGy & -11 & cGy & 98 & 0.3 \\
heart D33 & 1137 & cGy & 1174 & cGy & 37 & cGy & 155 & 0.15 \\
\hline \hline
\end{tabular}

Table 4.1 Mean differences between planning dose distribution and cumulative dose for the no-adapt simulation. Target coverage and normal-tissue dose metrics are included along with mean differences $(\Delta)$ and standard deviations $(\sigma)$ for each. Statistical significance as measured by two-tailed paired t-tests is indicated in the final column for differences between planned and no-adapt distributions. 


\section{Conclusion}

This thesis focused on quantifying the benefit of daily replanning for lung cancer patients and characterizing the tradeoffs of adaptive benefit with replanning frequency. In addition to these main objectives, a software tool was created to facilitate an adaptive paradigm by extending data objects and evaluation methods in the temporal domain. This provided a platform for conducting the research, and useful methods for evaluating planning data.

The goal of adaptive therapy is to improve targeting and spare normal tissues by dynamically modifying treatment in response to observed variation. Reductions in normal-tissue dose allow for target increases while maintaining acceptable levels of toxicity. Dose escalation in lung cancer is desirable as it increases the probability of local control.

Various studies have demonstrated dose sparing and associated escalation for an adaptive approach in lung cancer, and suggest an increased benefit with greater adaptive frequency; however, previous studies implement at most weekly replanning, leaving questions regarding the full potential of adaptive therapy.

Daily adaptation requires images for each replanning time-point to provide up-to-date anatomic information and a basis for dose calculation. In this work synthetic CT images were generated from an existing set of weekly images for a cohort of lung cancer patients using PCA. A novel method was implemented in the sampling process which preserved temporal trends such as tumor regression in the model and resulted in a set of image mappings that were used to map the first image in the weekly series. The use of synthetic images was advantageous because images and contours associated with a given time-point were based on a common mapping to the initial image resulting in consistency between images, contours, and dose volumes; additionally, 
extents of designated sub-clinical disease could be accurately tracked throughout treatment avoiding ambiguities regarding adequate target volumes for replanning time-points.

Daily replanning resulted in statistically significant dose decreases for all risk structures considered in this work. An average decrease in mean lung dose of 5\% allowed average increases in tumor dose of $441 \mathrm{cGy}$ when escalating to an isotoxic criteria, and escalation of approximately 17 Gy was achieved for a single patient. Cord tolerances were not exceeded for any escalation in target dose. These values were slightly less than those observed by other authors implementing lesser amounts of replanning frequency. In part the discrepancy may be a result of volumes to which dose was escalated. In this work dose was escalated to the whole of the CTV as opposed to boosting dose to the residual gross disease.

Additional planning studies were carried out that simulated a single adaptation at mid treatment and weekly replanning. Sequential comparison of simulations from the non-adaptive to the full-adaptive revealed incremental reductions that were statistically significant for both mean lung dose and mean esophageal dose revealing an increase in adaptive benefit with each increase in replanning frequency. Interestingly, the magnitude of benefit decreased as planning frequency increased with an average of $60 \%$ of mean lung dose reductions associated with daily replanning being achieved after a single mid-treatment replan, and $88 \%$ being realized after weekly replanning. Understanding tradeoffs between replanning frequency and adaptive benefit are important because resources are limited and replanning is currently expensive in terms of workload. Assuming that each instance of replanning is equally costly and that a given patient follows the averages stated above, four times the workload of weekly planning (i.e. 28 additional replans) would need to be expended to achieve the final $12 \%$ of potential benefit. In addition to boosting CTV as opposed to primary tumor, the trend of decreasing returns may help explain 
discrepancies between reported values of adaptive benefit in this thesis and the work reported by others.

A final effort was made to explore potential methods of identifying patients that may benefit from an adaptive approach. Correlations between reductions in mean lung dose and a variety of simple descriptors were investigated including: nodal status, initial PTV volume, and absolute change in PTV; of these, only the latter was found to be correlated with adaptive benefit. Relationships between patterns of target volume regression and adaptive benefit were investigated, noting that some irregularity (e.g. patterns of target volume regression followed by a trend of volume increase) seemed to be associated with patients that exhibited no adaptive benefit which suggests that temporal patterns may be important; however, sample size was not large enough to be conclusive.

Indicators of adaptive benefit are important to select which patients warrant allocation of additional resources inherent in replanning, and future work should be dedicated to this end. The software tool described in chapter 2 enables analysis of multiple signals that could be used in such efforts including temporal course of volume, mass, or centroid positions.

The major contributions of this work are 1) it identifies a need for tools suited to an adaptive paradigm and gives an example of such tools; 2) it introduces a novel method for sampling of synthetic geometries that preserves temporal anatomic trends providing a basis for adaptive studies; 3 ) demonstrates the benefits associated with daily adaptation; and 4) it characterizes the tradeoff between adaptive frequency and workload. These developments: provide insight into the value of adaptive radiation therapy for lung cancer, help inform decisions regarding it's implementation, and provide a basis for future studies. 


\section{References}

1. Heron M. Deaths: Leading Causes for 2009. National Vital Statistics Reports; 2012.

2. Siegel R, Naishadham D, Jemal A. Cancer statistics, 2013. CA Cancer J Clin 2013;63(1):11-30.

3. Halperin EC, Pérez CA, Brady LW. Perez and Brady's Principles and Practice of Radiation Oncology. Lippincott Williams \& Wilkins; 2008.

4. Howlader N, Noone AM, Krapcho M, Garshell J, Neyman N, Altekruse SF, et al. SEER Cancer Statistics Review, 1975-2010.

5. Mehta M, Scrimger R, Mackie R, Paliwal B, Chappell R, Fowler J. A new approach to dose escalation in non-small-cell lung cancer. Int $\mathrm{J}$ Radiat Oncol Biol Phys 2001;49(1):23-33.

6. Kong F-M, Haken Ten RK, Schipper MJ, Sullivan MA, Chen M, Lopez C, et al. Highdose radiation improved local tumor control and overall survival in patients with inoperable/unresectable non-small-cell lung cancer: Long-term results of a radiation dose escalation study. International Journal of Radiation Oncology*Biology*Physics 2005;63(2):324-33.

7. Steenbakkers RJHM, Duppen JC, Fitton I, Deurloo KEI, Zijp LJ, Comans EFI, et al. Reduction of observer variation using matched CT-PET for lung cancer delineation: A three-dimensional analysis. International Journal of Radiation Oncology*Biology*Physics 2006;64(2):435-48.

8. Van de Steene J, Linthout N, de Mey J, Vinh-Hung V, Claassens C, Noppen M, et al. Definition of gross tumor volume in lung cancer: inter-observer variability. Radiother Oncol 2002;62(1):37-49.

9. Giraud P, Antoine M, Larrouy A, Milleron B, Callard P, De Rycke Y, et al. Evaluation of microscopic tumor extension in non-small-cell lung cancer for three-dimensional conformal radiotherapy planning. Int J Radiat Oncol Biol Phys 2000;48(4):1015-24.

10. de Boer HC, van Sörnsen de Koste JR, Senan S, Visser AG, Heijmen BJ. Analysis and reduction of $3 \mathrm{D}$ systematic and random setup errors during the simulation and treatment of lung cancer patients with CT-based external beam radiotherapy dose planning. Int $\mathbf{J}$ Radiat Oncol Biol Phys 2001;49(3):857-68.

11. Keall PJ, Mageras GS, Balter JM, Emery RS, Forster KM, Jiang SB, et al. The management of respiratory motion in radiation oncology report of AAPM Task Group 76. 2006.

12. Stevens CW, Munden RF, Forster KM, Kelly JF, Liao Z, Starkschall G, et al. Respiratory- 
driven lung tumor motion is independent of tumor size, tumor location, and pulmonary function. Int J Radiat Oncol Biol Phys 2001;51(1):62-8.

13. Kupelian PA, Ramsey C, Meeks SL, Willoughby TR, Forbes A, Wagner TH, et al. Serial megavoltage CT imaging during external beam radiotherapy for non-small-cell lung cancer: Observations on tumor regression during treatment. International Journal of Radiation Oncology*Biology*Physics 2005;63(4):1024-8.

14. Britton KR, Starkschall G, Tucker SL, Pan T, Nelson C, Chang JY, et al. Assessment of Gross Tumor Volume Regression and Motion Changes During Radiotherapy for NonSmall-Cell Lung Cancer as Measured by Four-Dimensional Computed Tomography. International Journal of Radiation Oncology*Biology*Physics 2007;68(4):1036-46.

15. van Zwienen M, van Beek S, Belderbos J, van Kranen S, Rasch C, van Herk M, et al. Anatomical changes during radiotherapy of lung cancer patients. Int J Radiat Oncol Biol Phys 2008;72(1):S111.

16. ICRU. Prescribing, recording, and reporting photon beam therapy. Bethesda: International Commission on Radiation Measurements; 1993.

17. Yeung AR, Li JG, Shi W, Newlin HE, Chvetsov A, Liu C, et al. Tumor localization using cone-beam CT reduces setup margins in conventionally fractionated radiotherapy for lung tumors. Int J Radiat Oncol Biol Phys 2009;74(4):1100-7.

18. Bissonnette J-P, Purdie TG, Higgins JA, Li W, Bezjak A. Cone-beam computed tomographic image guidance for lung cancer radiation therapy. Int $\mathrm{J}$ Radiat Oncol Biol Phys 2009;73(3):927-34.

19. Yan D, Vicini F, Wong J, Martinez A. Adaptive radiation therapy. Phys Med Biol 1997;42(1):123.

20. Yan D, Wong J, Vicini F, Michalski J, Pan C, Frazier A, et al. Adaptive modification of treatment planning to minimize the deleterious effects of treatment setup errors. Int $\mathrm{J}$ Radiat Oncol Biol Phys 1997;38(1):197-206.

21. Yan D, Lockman D, Brabbins D, Tyburski L, Martinez A. An off-line strategy for constructing a patient-specific planning target volume in adaptive treatment process for prostate cancer. Int J Radiat Oncol Biol Phys 2000;48(1):289-302.

22. Lof J, Lind BK, Brahme A. An adaptive control algorithm for optimization of intensity modulated radiotherapy considering uncertainties in beam profiles, patient set-up and internal organ motion. Phys Med Biol 1998;43(6):1605-28.

23. Zerda A de L, Armbruster B, Xing L. Formulating adaptive radiation therapy (ART) treatment planning into a closed-loop control framework. Phys Med Biol 2007;52(14):4137-53.

24. Martinez AA, Yan D, Lockman D, Brabbins D, Kota K, Sharpe M, et al. Improvement in 
dose escalation using the process of adaptive radiotherapy combined with threedimensional conformal or intensity-modulated beams for prostate cancer. Int J Radiat Oncol Biol Phys 2001;50(5):1226-34.

25. Hugo GD, Yan D, Liang J. Population and patient-specific target margins for 4D adaptive radiotherapy to account for intra- and inter-fraction variation in lung tumour position. Phys Med Biol 2007;52(1):257-74.

26. Harsolia A, Hugo GD, Kestin LL, Grills IS, Yan D. Dosimetric Advantages of FourDimensional Adaptive Image-Guided Radiotherapy for Lung Tumors Using Online ConeBeam Computed Tomography. International Journal of Radiation Oncology*Biology*Physics 2008;70(2):582-9.

27. Ramsey CR, Langen KM, Kupelian PA, Scaperoth DD, Meeks SL, Mahan SL, et al. A technique for adaptive image-guided helical tomotherapy for lung cancer. Int J Radiat Oncol Biol Phys 2006;64(4):1237-44.

28. Woodford C, Yartsev S, Dar AR, Bauman G, Van Dyk J. Adaptive Radiotherapy Planning on Decreasing Gross Tumor Volumes as Seen on Megavoltage Computed Tomography Images. International Journal of Radiation Oncology*Biology*Physics 2007;69(4):131622 .

29. Guckenberger M, Wilbert J, Richter A, Baier K, Flentje M. Potential of adaptive radiotherapy to escalate the radiation dose in combined radiochemotherapy for locally advanced non-small cell lung cancer. Int J Radiat Oncol Biol Phys 2011;79(3):901-8.

30. Hugo GD, Weiss E, Badawi A, Orton M. Localization accuracy of the clinical target volume during image-guided radiotherapy of lung cancer. Int J Radiat Oncol Biol Phys 2011;81(2):560-7.

31. Guckenberger M, Richter A, Wilbert J, Flentje M, Partridge M. Adaptive radiotherapy for locally advanced non-small-cell lung cancer does not underdose the microscopic disease and has the potential to increase tumor control. Int J Radiat Oncol Biol Phys 2011;81(4):e275-82.

32. Yan D, Jaffray DA, Wong JW. A model to accumulate fractionated dose in a deforming organ. Int J Radiat Oncol Biol Phys 1999;44(3):665-75.

33. Marks LB, Bentzen SM, Deasy JO, Kong F-MS, Bradley JD, Vogelius IS, et al. Radiation dose-volume effects in the lung. Int J Radiat Oncol Biol Phys 2010;76(3 Suppl):S70-6.

34. Nioutsikou E, Webb S, Panakis N, Bortfeld T, Oelfke U. Reconsidering the definition of a dose-volume histogram. Phys Med Biol 2005;50(11):L17-9.

35. Jaffray DA, Lindsay PE, Brock KK, Deasy JO, Tomé WA. Accurate accumulation of dose for improved understanding of radiation effects in normal tissue. Int J Radiat Oncol Biol Phys 2010;76(3 Suppl):S135-9. 
36. Christensen GE, Song JH, Lu W, Naqa El I, Low DA. Tracking lung tissue motion and expansion/compression with inverse consistent image registration and spirometry. Med Phys 2007;34(6):2155-63.

37. Weiss E, Fatyga M, Wu Y, Dogan N, Balik S. Dose Escalation for Locally Advanced Lung Cancer Using Adaptive Radiation Therapy With Simultaneous Integrated VolumeAdapted Boost. Int J Radiat Oncol Biol Phys 2013; 
Appendix I

Treatment History Browser

User's Guide 


\section{Treatment History Browser User Guide}

Christian Dial

February 15, 2014 


\section{CONTENTS}

$\begin{array}{ll}\text { INTRODUCTION } & 1\end{array}$

$\begin{array}{ll}\text { Software requirements and dependencies } & 1\end{array}$

Package contents and installation 2

DATA STRUCTURES

USER INTERFACE 6

$\begin{array}{ll}\text { Start Window } & 6\end{array}$

$\begin{array}{ll}\text { Region of Interest Group Editor } & 7\end{array}$

$\begin{array}{lr}\text { Treatment Editor } & 9\end{array}$

Main Window 11 


\section{INTRODUCTION}

Traditional methods of radiation therapy planning typically utilize CT images associated with a single time-point acquired before treatment begins, and treatment planning systems (TPS) currently reflect this "static" workflow. Adaptive radiotherapy (ART) is a treatment strategy that utilizes information acquired throughout the course of delivery to optimize target volumes and reduce treatment margins. If additional volumetric imaging is performed, re-planning may be carried out to accommodate inter-fraction variation; however, clinically this is implemented on a very limited basis when large and acute changes are observed that may significantly affect the quality of the plan. Routine implementation of adaptive planning at multiple treatment timepoints is currently an area of active research and represents a shift in the traditional treatment paradigm extending planning and evaluation into the temporal domain.

The Treatment History Browser (THB) is a software tool that facilitates implementation of ART by likewise extending existing TPS functionality in a temporal dimension. It is implemented as a plugin to the Pinnacle TPS (Philips Oncology, Fitchburg, WI) using the python scripting language and the Qt GUI toolkit, and is intended as a research tool to organize, conduct, navigate, and evaluate ART studies.

The tool was created as part of thesis work performed at Virginia Commonwealth University titled "Adaptive radiation therapy for lung cancer." As part of this research, synthetic datasets were generated for each fraction of a typical treatment regimen (35 fractions) resulting in a large number of displacement vector fields (DVF), images, contours, and trials. To efficiently store and manage the data a dynamic approach was taken in which DVFs were stored to disk and all other data was generated as needed on demand. While dynamic data need not be utilized, the management and creation of synthetic datasets is integrated into the tool's functionality.

Methods for displaying temporal signals are implemented as an alternative to cumulative approaches that sum dose to a reference dataset. Temporal signals include conventional dosevolume metrics (e.g. D95) tracked for each fraction of treatment, dose statistics, volume, mass, centroid position, and composite fraction dose volume histograms (DVH).

This guide is intended for researchers that wish to make use of the tool in its current state, extend its functionality, or use it as an example and starting point for creating GUI plugin applications for the Pinnacle TPS using python scripting and the Qt GUI toolkit.

\section{Software requirements and dependencies}

The THB was developed as a plugin for the Pinnacle TPS and has only been tested with the following configuration:

- Pinnacle TPS version 9.100 with python scripting plugin

- Python 2.7 (Pinnacle specific build)

- PyQt4 (Python bindings for the Qt GUI toolkit)

- numpy (Python package for scientific computing) 
Plotting functionality utilizes xmgrace as a backend to produce .png image files and must be installed on the workstation in use.

\section{Package contents and installation}

The THB is a package of several python scripts and other resources that reside in a folder titled "txhsbr2.0" which contains the following directories and files:

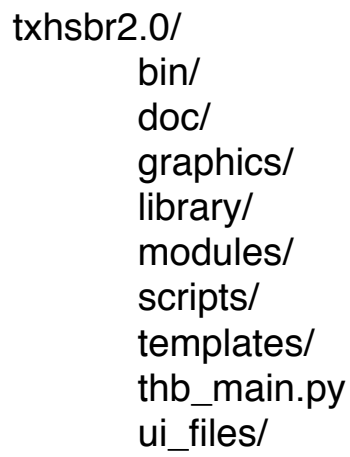

The single python script that resides at this level is "thb_main.py" and is initiated using the python script-tree plugin from within Pinnacle to start the application. Modules that reside in the different subdirectories are referenced relative to the location of the main script file, thus the package directory may reside at any location on disk and may be moved at anytime without

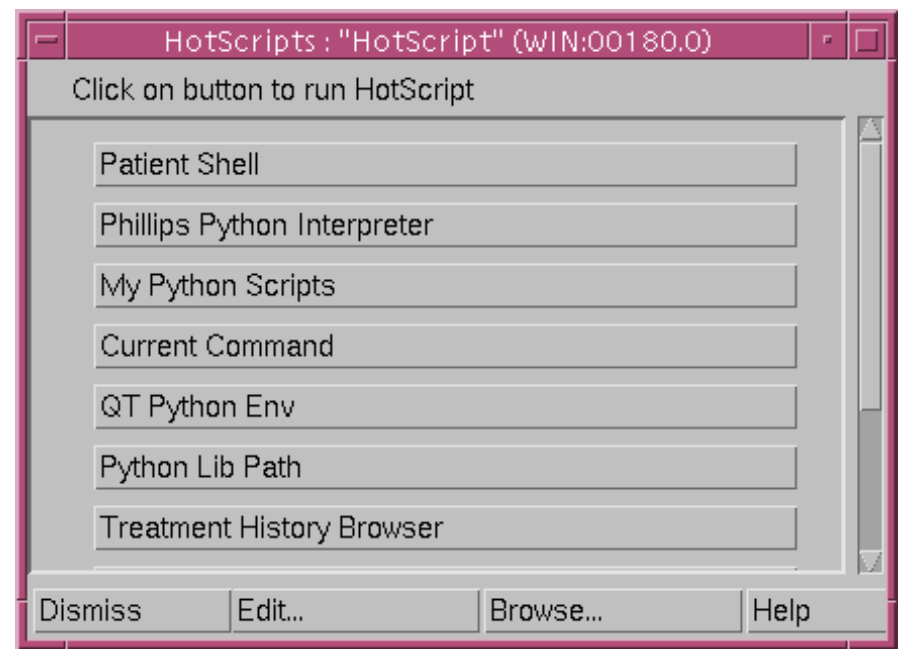

disrupting the applications functionality. Python is an interpreted language thus no compiling is necessary in the installation process for the core functionality of the application.

After ensuring that the preliminary requirements described in the previous section are met, installation consists of simply copying the folder "txhsbr2.0" to disk. As an alternative to locating and running the "thb_main.py" file to initiate the application, a traditional Pinnacle script may be written to invoke the main application file, and incorporated into the user's hot-script list. 
Apart from the "thb_main.py" file, the application directory contains various divisions of resources whose contents are outlined here.

\section{directory contents}

bin/ Though the core functionality is written in pure python, certain tasks may require the efficiency of compiled code. Such utilities are stored here.

doc/ Contains class documentation for core python modules and scripts for automatically generating the same using a python module named Sphinx.

graphics/ Contains all icon and graphics files included in the user interface.

library/ A collection of modules that are integral to the application's functionality but that operate independent of it and may be utilized in other applications.

modules/ Core application code for each of the application windows.

scripts/ User defined scripts to automate the applications functionality.

templates/ A group of templates for quickly setting up common treatments (e.g. adapt every week, adapt every day)

ui_files/ Files that specify the appearance and behavior of the user interface most of which are automatically generated using Qt Designer (Digia Plc, Helsinki Finland) to create .ui files that then may be converted to python code using the "pyside-uic" utility. 


$$
\text { tx [ 'Adapt'] }
$$

returns the treatment object named "Adapt"

$$
t x[\text { 'Adapt']. } \mathrm{fx}[1]
$$

returns the first fraction object associated with this treatment and

$$
\operatorname{tx}[\text { 'Adapt']. } \mathrm{fx}[1] \cdot \text { date }
$$

returns the date associated with this fraction object.

\section{ROI group object}

Like trials, ROls correspond to a single image and in a traditional paradigm are static representations of dynamic structures. Incorporating multiple images into the planning process necessitates an additional object to manage all ROls associated with a given anatomical structure. ROI group objects inherit from standard python lists and incorporate the following additional attributes.

\section{attribute type description}

color string color to be used for ROls in group; all ROls are set to this color when displayed in Pinnacle and signals associated with the group are plotted in the same color

display bool designates whether the group as a whole should be displayed in Pinnacle

signals list lists all signals associated with ROI group (e.g. volume, mass, etc.) that may be plotted in the application. 


\section{DATA STRUCTURES}

To accommodate large sequences of temporal data that correspond to multiple treatment timepoints, various new data structures are created to extend the existing functionality of the TPS. In Pinnacle, the basic objects of planning and evaluation that correspond to single images are that of a trial and a region of interest (ROI). Traditionally, plans associated with trial objects are delivered for the duration of treatment and thus trials correspond to a treatment regimen. In an adaptive paradigm, plans associated with trial objects may correspond to a single fraction or a subset of fractions. Having a single object that may refer to an entire treatment or a subset of fractions creates an ambiguity that is resolved by introducing "treatment" and "fraction" objects to reflect the dynamic planning workflow. Single ROls are grouped together into "ROI group" objects.

\section{Fraction object}

Fraction objects correspond to a single treatment time-point and are associated with a single trial object. Multiple fractions may refer to the same image and trial, allowing flexibility in the number of images incorporated into the planning process and the amount of re-plans carried out.

Fraction object attributes are as follows:

$\begin{array}{lll}\text { attribute } & \text { type } & \text { description } \\ \text { image } & \text { string } & \text { name of image used to represent the given fraction } \\ \text { trial } & \text { string } & \text { name of pinnacle trial object associated with the fraction } \\ \text { date } & \text { date } & \text { date of treatment associated with the fraction } \\ \text { adapt } & \text { bool } & \text { value to specify whether re-planning will be carried out for the fraction }\end{array}$

\section{Treatment object}

Treatment objects provide a way to organize a set of fractions and are the temporal analogue of trials. The main attribute of the treatment object is a python dictionary named " $f x$ " whose keys correspond to the fraction number and whose values are the fraction objects themselves. A treatment object may consist of an arbitrary amount of fractions each of which may correspond to different or the same images i.e. multiple fractions may be represented by a single image. Using a dictionary to organize the fraction object provides a natural and readable way to access fraction attributes.

Multiple treatment objects may be created to evaluate and compare multiple treatment courses. An additional python dictionary named "tx" (an attribute of the main object) is implemented to contain the different treatment objects. Nesting multiple dictionary objects in this way results in a convenient and readable method to access each of the objects. For example, 


\section{USER INTERFACE}

\section{Start Window}

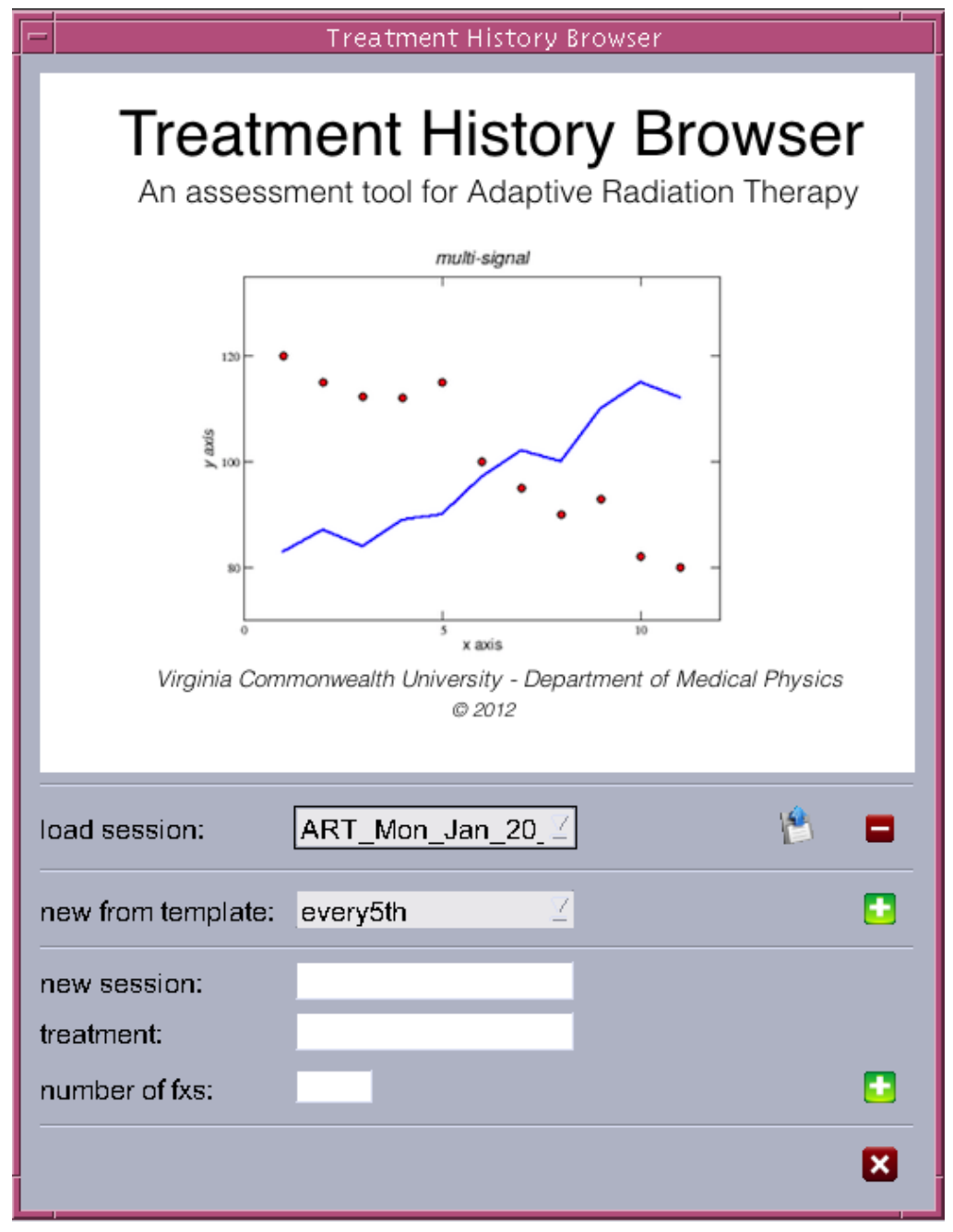

All data associated with a given THB instance constitutes a "session" and is stored in a single file with the extension ".thb" in a directory titled "TxHsBr" which resides in the plan directory for a given patient. Each time the THB is invoked, the start window is presented to the user and facilitates the creation, loading, and management of session files. All .thb files located in the $\mathrm{TxHsBr}$ directory appear in the "load session" drop down box and may be deleted or loaded from this form. An additional drop-down box lists templates that are found in the "templates" folder of the application directory. Templates specify a specific setup for a given session (e.g. treatment, number of fractions, specification of adaptive fractions etc.) and provide a convenient method for initiating common regimens. Alternatively, a new session may be created by entering a name and setting up at least one treatment in the entry fields in the bottom portion of the form. 


\section{Region of Interest Group Editor}

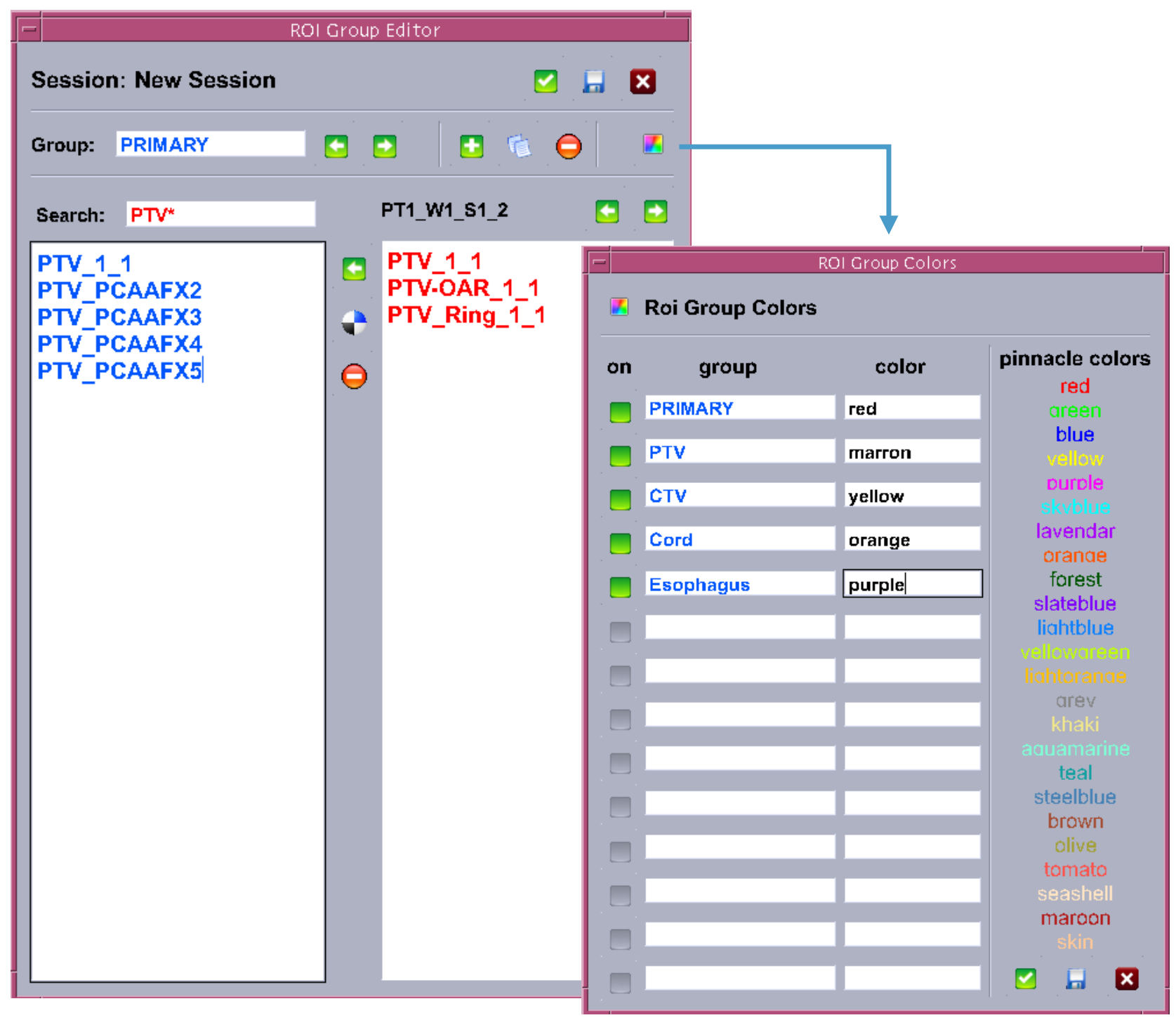

\section{Group Management}

The "ROI Group Editor" form is presented after creating a new session or may be accessed via the "utilities" menu on the "Main Window" form (see Main Window) and is used to manage all $\mathrm{ROI}$ groups. The session name is displayed at the top left, and at the top right of the form a "check" button, "save" button, and "cancel" button are used to save and exit, save without exiting, and exit without saving respectively. The current group name appears in the "Group" box under the session name and may be edited directly. Adjacent to the "Group" box are 6 action buttons including: left and right arrow buttons for cycling through existing groups; an "add" button for group creation; a "copy" button for duplicating the current group; a "delete" button for removing the current group; and a "color" button to bring up a separate form for specifying group colors (right form above). 
ROls that pertain to the current group are managed using the bottom portion of the form. ROls included in the current group are listed on the left in blue and search results are listed in red on the right. Each list is directly editable and supports copy and paste functions. Each new line represents an entry, and any valid entry (i.e. that matches the name of an ROI in Pinnacle) listed on the left will be added to the group.

ROls may be added by manually entering names in the left list or by using the search functionality of the form. Queries are carried out using the "Search" box on the left or the image navigation on the right. "Search" box queries support Unix-style wildcards to return ROls that match the specified criteria (e.g. "PTV*1" would return a list of all ROls whose names begin with "PTV" and end with "1"). Image queries return all ROls that reside on the listed image and are conducted by using the left and right arrow keys to cycle through Pinnacle volumes. After a search is conducted search results appearing on the right in red may be incorporated into the current group by copying and pasting or by using the left arrow button between the two lists. This button will transfer all entries on the right to the current ROI group and they will then appear in blue on the left. If either query returns results that the user wishes to exclude these may be manually deleted from the right list before transferring them to ROI group.

To remove ROls from a group, select them on the left and delete them manually or by pushing the "update" button (black, white, and blue pinwheel between the two lists) which will remove any highlighted text. Alternatively, the "delete" button between the two lists will clear all ROI entries on the left.

\section{Primary ROI group}

For sessions that utilize dynamic data (i.e. propagation of image volumes and contours), a "PRIMARY" group must be populated and is used to specify those contours that should be included in propagation. The user is presented with the ROI group form after creating a new session with the "PRIMARY" group waiting to be populated; when a session is created using a template the "PRIMARY" group is specified in the body of the script and the ROI group form is bypassed. The "PRIMARY" group is accessible in the ROI group editor form however it is not included in the ROI groups used for querying image derived data and likewise is excluded from all other forms. 


\section{Treatment Editor}



The "Treatment Editor" form displays all data related to a given treatment and facilitates management of all treatment objects pertaining to the session and, like the "ROI Group Editor" form, is presented to the user upon creating a new session unless a session template is used. The "Treatment" box specifies the current treatment name and is directly editable with left and right arrow keys for navigating between treatment objects. Buttons for adding, copying, and removing treatments reside to the right of the navigation arrows; the "check" button at the top left saves and exits the form, while the adjacent "cancel" button exits without saving. All fraction 
objects corresponding to the selected treatment are represented as are each of the fraction attributes. Image name, whether the image is synthetic, trial name, whether the trial is adaptive, fraction date, and (for synthetic data) the DVF used to propagate the primary image and contours are listed. Image names should correspond to existing Pinnacle volumes or be associated with an existing DVF according to Pinnacle naming conventions (e.g.

PT1W1S1_PCAAFX35_Demons_10.dvf) and treatments may consist of any mixture of synthetic and existing images. Trial names are automatically generated based on image volume and whether re-planning will be carried out for the fraction.

Though data may be entered manually, various autofill features are implemented to facilitate treatment setup. When the "auto fill" check box is checked auto completion for images and dates become active. For images, fraction two entries are used as a template and all subsequent image names are entered with incremented numbers (e.g. entering "PCAAFX2" autofills subsequent fractions with PCAAFX3, PCAAFX4, etc.). As an alternative, the "Pinnacle Img Entry" section allows quick entry of existing images. Up and down arrow keys move up and down the image section of the form and left and right arrows cycle through existing images. Dates are entered by clicking on the arrow to the shadowed-arrow at the right of each date box to reveal a current calendar. When "auto fill" is checked, this results in automatically setting dates to subsequent business days. After autofilling, manual modifications may still be made.

Adaptive schedules are set by selecting the "adapt" check boxes next to each trial entry. This may be done manually or by selecting a pre-defined schedule from the "adapt" drop-down box next to the "auto fill" check box.

After images, dates, and adaptive schedules have been set, the form should be updated so that trial and DVF names reflect the new entries. This is accomplished by pushing the "update" button next to the "adapt" drop-down box (blue, white, and black pinwheel). 


\section{Main Window}

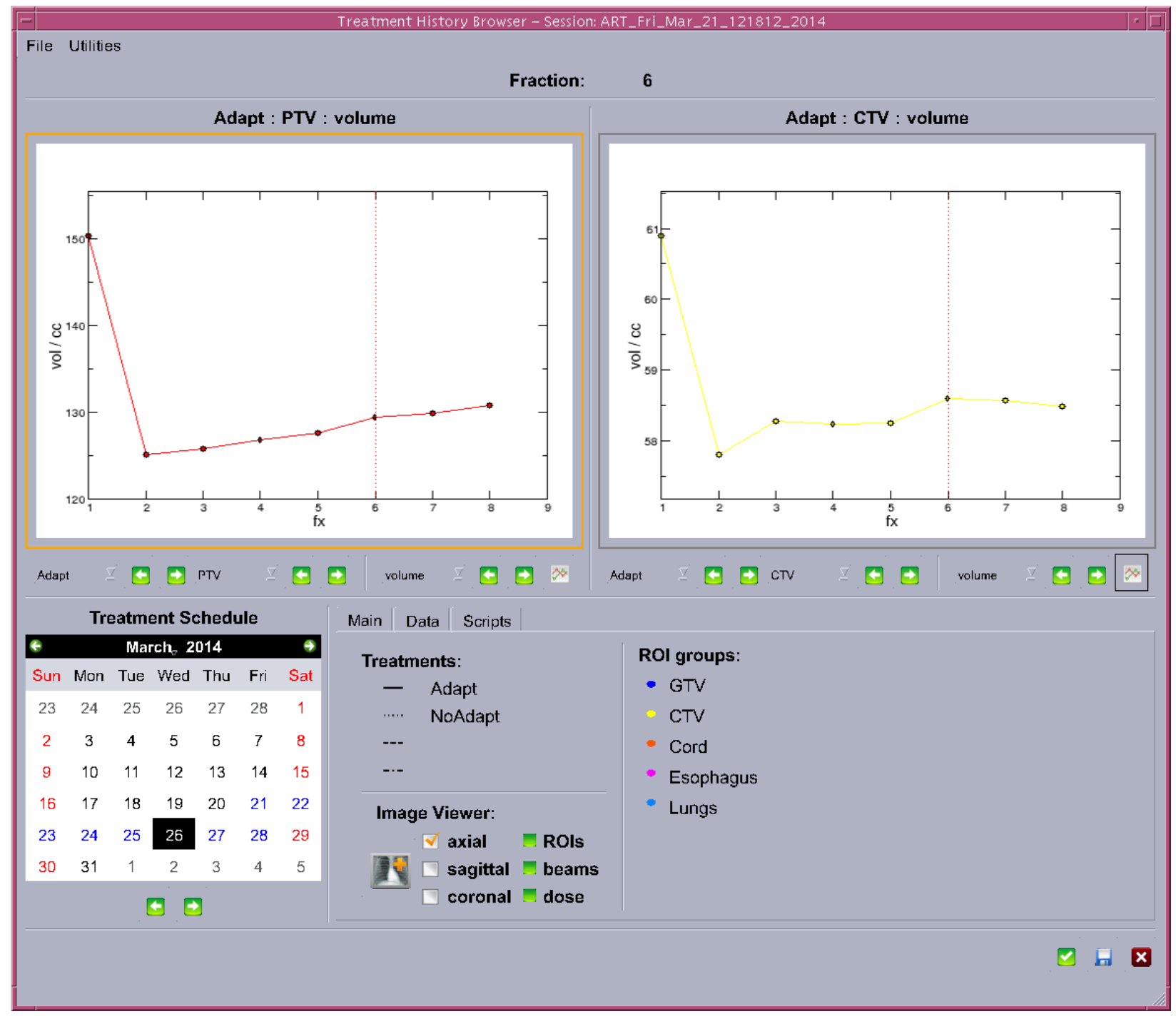

\section{Basic functionality}

After a session has been set up, the main window is initiated and presented to the user. The central features of the application are two large plotting areas that are used to display the different temporal and fraction based signals supported by the tool. The two plotting areas have the same functionality but operate independently and are utilized for comparisons between different signals. Temporal signals include: ROI volume, mass, centroid magnitude deviation, and arbitrary dose volume parameters (e.g. dose to $95 \%$ of treatment volume) all of which are plotted as a function of fraction number. Additional signals include individual fraction DVHs and composite DVHs that display all individual fraction DVHs on a single plot.

Multiple signals associated with multiple ROls and treatments represents a large amount of data that may be conceptualized as a multi-dimensional signal space with treatment, fraction, ROI, and signal, representing the distinct dimensions. A primary purpose of the tool was to provide a 
convenient method for navigating the large signal space and evaluating different treatments. For each dimension, with the exception of treatment fraction, a drop-down box displays the currently selected treatment, $\mathrm{ROI}$, or signal respectively and allows the user to navigate quickly to a specific portion of the space. Additionally, left and right arrow keys are used to decrement or increment along each dimension respectively. The current selection of treatment, ROI group and signal are specified above the plot area.

Treatment fractions may be navigated by selecting the associated day on the treatment calendar which has treatment days highlighted in blue or by using the arrow keys to increment and decrement treatment fraction. For the current selection, data is displayed by clicking the plot icon at the lower right of each plot area. Alternatively, an interactive mode may be activated by left-clicking in the plot area which reveals an orange border to indicate that interactive mode is set. In interactive mode, any change to the current selection automatically updates the plot

area. Interactive mode is turned off by left-clicking in the plot area which clears the orange border. Fraction number is displayed at the top of the form and is indicated in time-series plots by a dotted red line; in multi-fraction DVH plots, the current fraction is highlighted in bold.

Signals are plotted by first retrieving data from the Pinnacle application and storing it in the signal data-object. Before data retrieval, the signal dictionary is checked for the relevant information and if it is found it is loaded without an additional request from Pinnacle. This enhances responsiveness of the tool but allows for the possibility that displayed data does not reflect the most recent data in Pinnacle if anything is modified. To force update before plotting, the user may select the "update plot" option from the contextual menu by first right-clicking in the plot area.

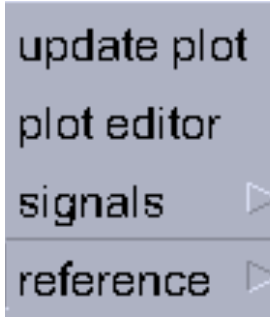

If the user wishes to modify or save a plot, an external plot editor may be invoked by selecting "plot editor" from the context menu.

\section{Signal addition and selection}

As mentioned, the tool allows the user to specify arbitrary dose-volume signals for plotting. These are specified by selecting "add dose or volume metric" under "signals" from the contextual menu.

\begin{tabular}{l|l}
$\begin{array}{l}\text { Update plot } \\
\text { plot editor }\end{array}$ & \\
\hline signals & add dose or volume metric \\
reference & set ROl group signals
\end{tabular}


A "add signal" dialogue then appears and the user is prompted to specify the new signal by entering "D" or "V" for dose and volume respectively followed by a number to specify the percentage of the volume (in the case of dose metrics) or the dose in Gray (in the case of volume metrics).

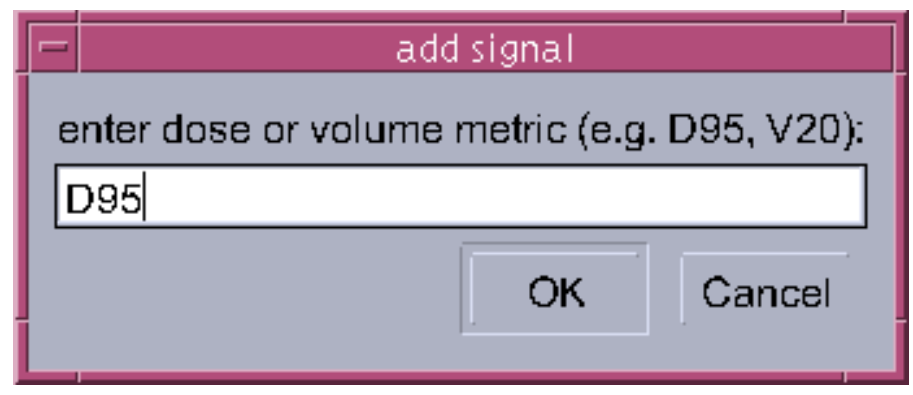

Another useful feature of the tool is the ability to specify a set of signals for each ROI group. This allows efficient navigation of only relevant signals for each ROI. Specifying ROI group signals is done by selecting "set ROI group signals" from the "signal" portion of the contextual menu. A checkbox dialogue is then revealed for selection and signal drop-downs are repopulated based on the specific ROI group selected.

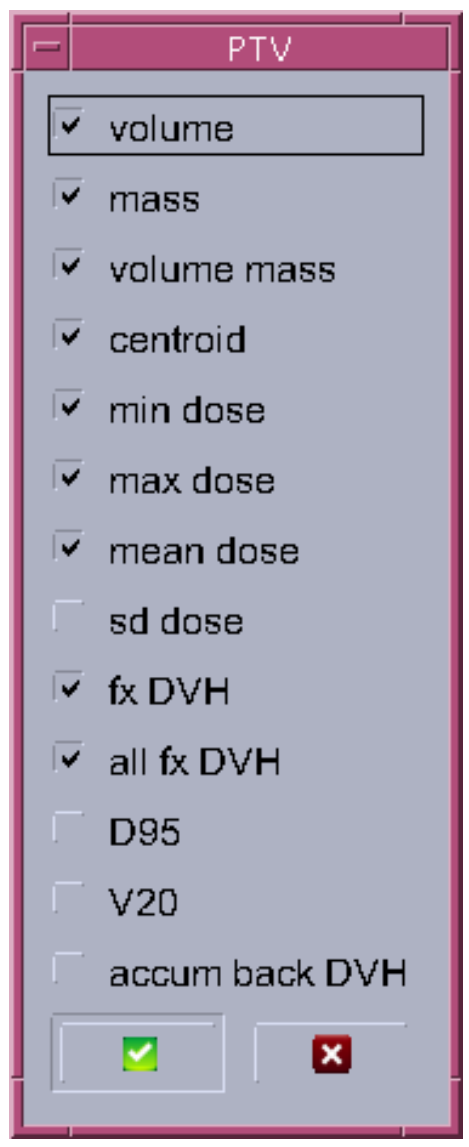




\section{Reference data}

While the tool may be used to interactively navigate individual signals, multiple treatments and/ or ROls may be specified as reference data and will be plotted in addition to the selected signal. Reference data is specified by right clicking in the plot area to reveal the contextual menu and is specific to each area (i.e. different reference data may be set for the different plots). Under the "reference" section, treatments, time-series ROI groups, and DVH ROI groups may be specified.

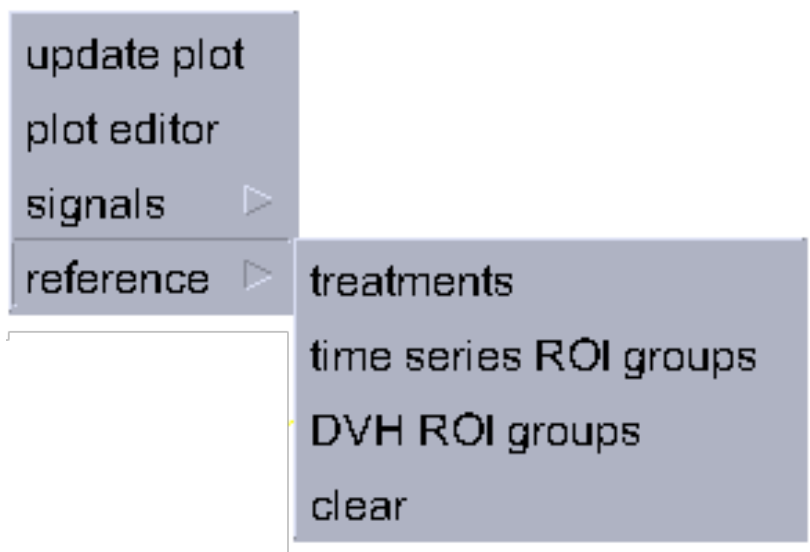

Each selection reveals a checkbox dialogue for selecting the reference data.

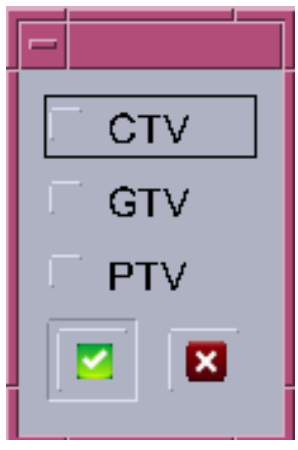

To remove reference data, groups or treatments may be deselected manually or by selecting the "clear" option under "reference."

\section{Main tab}

Next to the calendar is a tab section containing the "Main", "Data", and "Scripts" tabs. The "Main" tab is the default tab and appears when the Main Window is initiated. It contains a legend for signal data and a section for controlling a basic image viewer. 


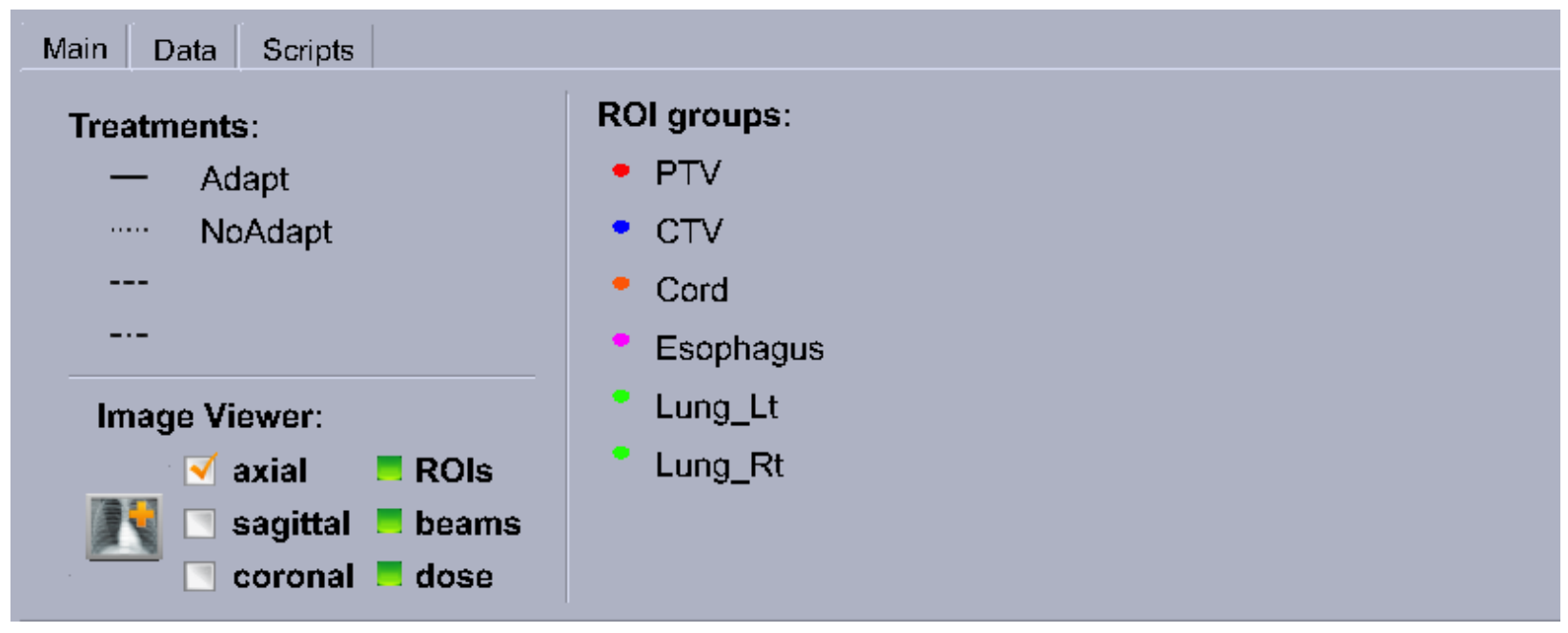

To distinguish between different treatments and ROIs, line-styles and colors are assigned respectively and are held constant to avoid ambiguity in plots. Colors may be adjusted using the ROI group color dialogue accessible via the Utilities drop-down menu at the top of the main form or through the ROI Group Editor; line-styles are assigned sequentially and automatically as new treatments are added and are not modifiable by the user.

Clicking the image view icon (image of CT scan with orange plus) creates a new Pinnacle image view instance that is controlled by the software tool. The image view can have 1 to 3 windows each associated with axial, sagittal, and coronal views respectively. The major convenience of the image view is that it reflects the current fraction selection and can be used in conjunction with the plot data to effectively evaluate fraction data.

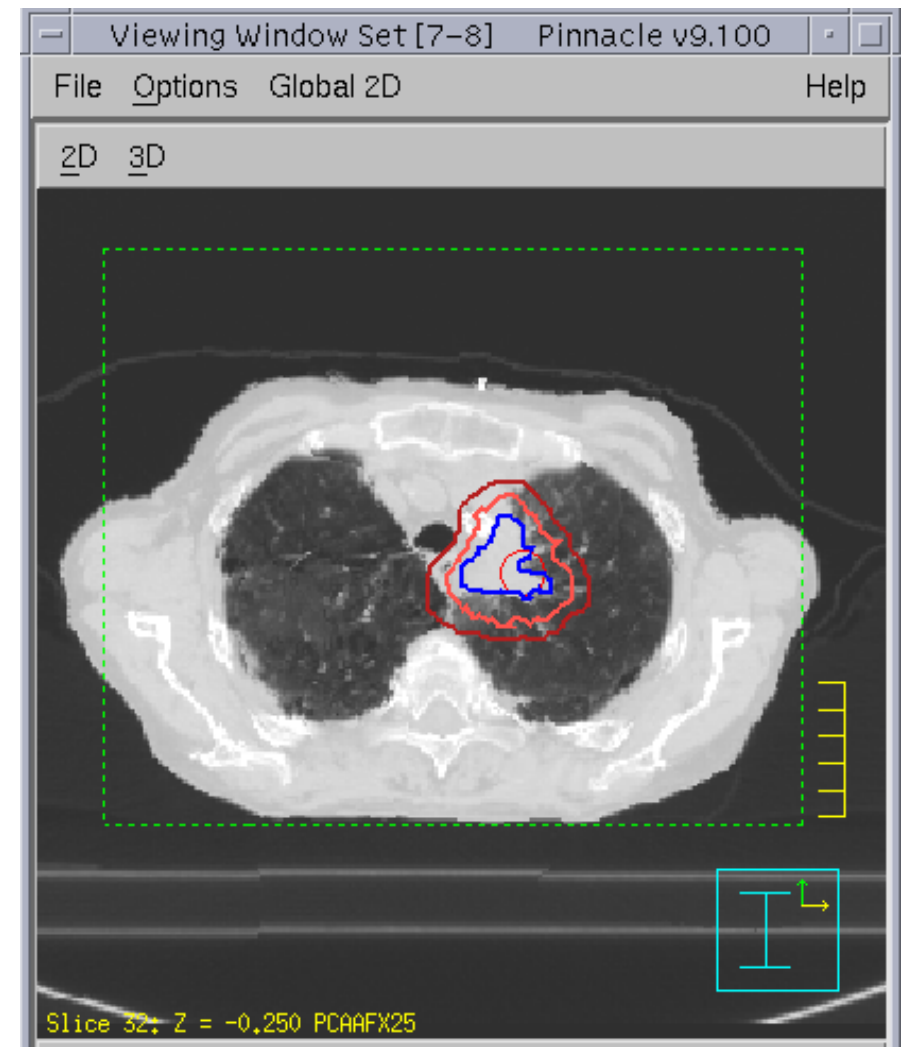




\section{Data tab}

The "Data" tab is used to summarize and manage treatment data. The currently selected treatment is displayed in the drop-down box at the top of the form and image and trial names associated with each fraction are listed in the table below. Blue text indicates that the image or trial currently exists in Pinnacle while grey text indicates that it is not currently found in Pinnacle (e.g. synthetic image names appear grey in the table until they are generated). Adaptive fractions are indicated by an orange fill.

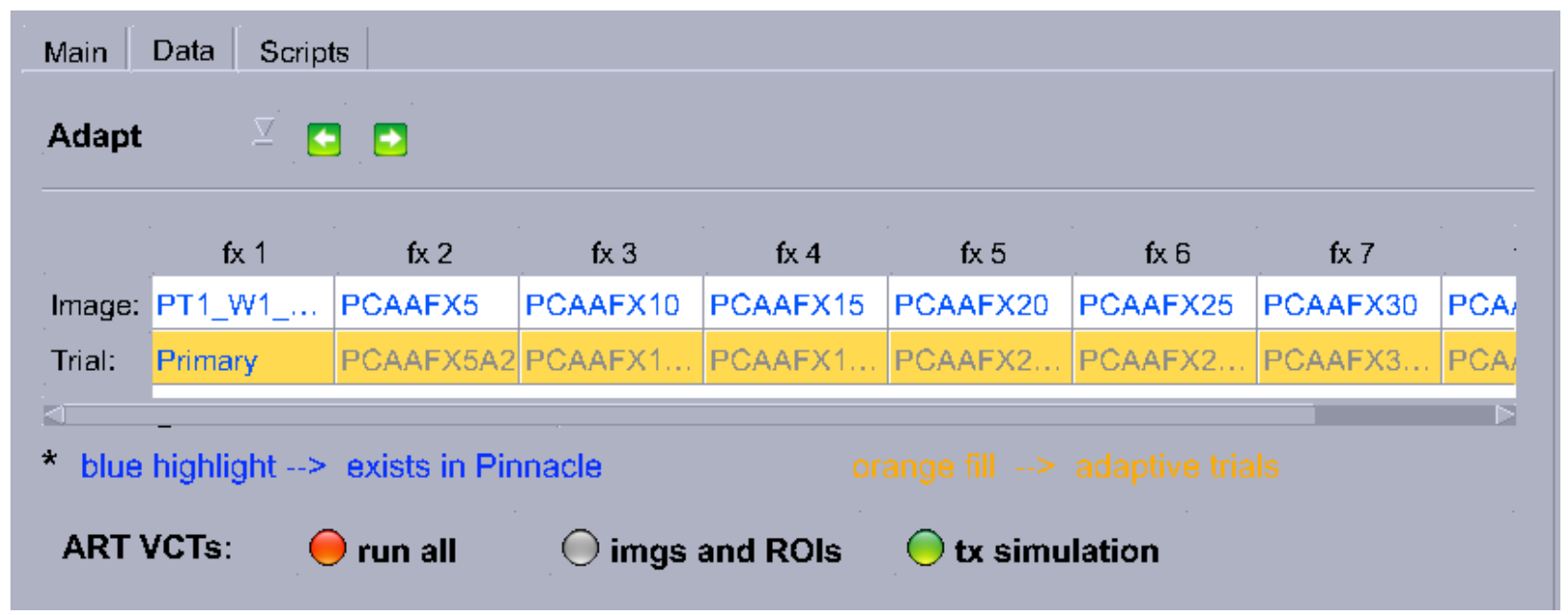

In addition to data summary, creation of synthetic images and automatic treatment simulations are initiated using this tab. The grey "imgs and ROls" button creates all synthetic images using the associated DVFs previously specified in the treatment setup and propagates all contours in the "PRIMARY" ROI group using the same.

The green "tx simulation" button carries out an adaptive simulation based on the adaptive schedule specified in treatment setup. For adaptive fractions the most recent set of beams and apertures are copied to the trial and are re-optimized based on the corresponding planning target volume (PTV). For non-adaptive plans, beams and apertures are copied and dose recalculated. 


\section{Scripts tab}

The scripts tab provides a column view of the path hierarchy for locating and calling scripts; calls are made by double clicking the the file name.

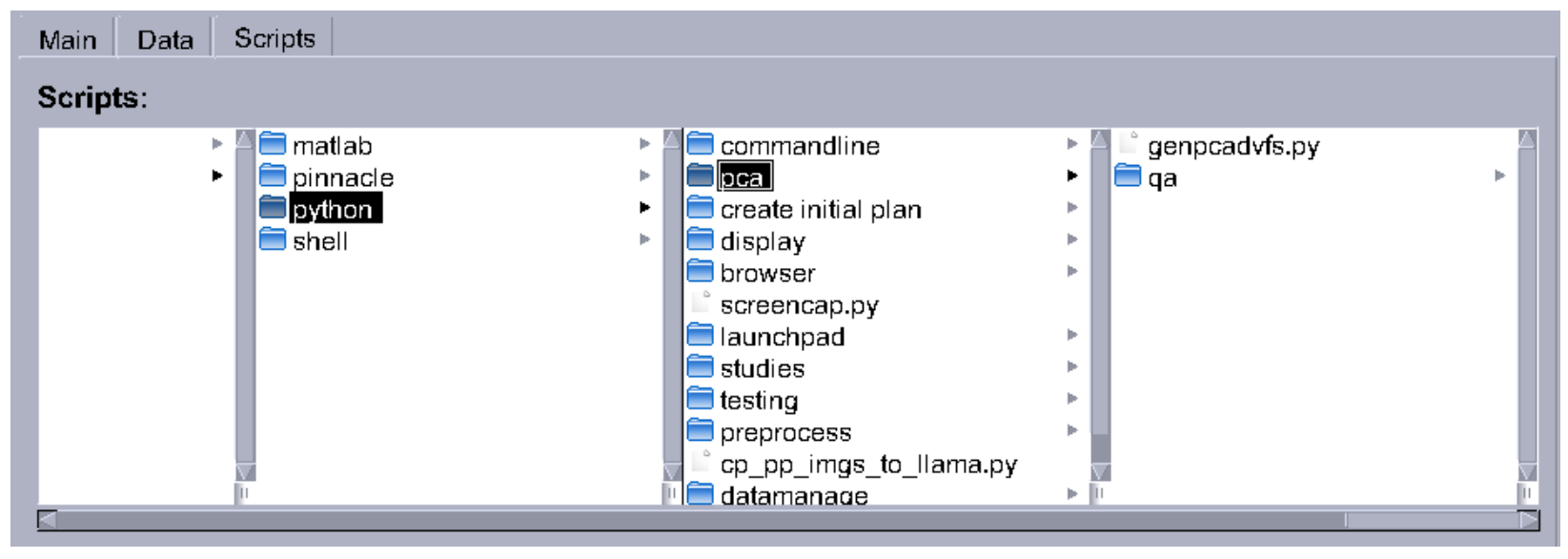




\section{Treatment History Browser Class Documentation}

Christian Dial

February 15, 2014 


\section{CONTENTS}

pinnLib MODULE

thb_database MODULE 15

thb_mainwindow $\quad 21$ 


\section{PINNLIB MODULE}

pinnLib.accumWindow (PinObj)

pinnLib. accumulateDose (PinObj, trials, tgtimg, atrl)

Description: accumulates dose from trials in list trials to img tgtimg and renames accumlated trial to atril

pinnLib . addBeam $($ PinObj, beam, angle $=$ None, poi=None $)$

Description: adds beam to current trial with name beam, gantry angle angle, and centered on point poi

pinnLib.addobjectives (PinObj, $n$ )

Description: add $n$ research objectives

pinnLib.addPoI (PinObj, poi, roi=None)

Description: adds POI with name poi; if roi given, poi is placed at center or ROI roi

pinnLib.addROIs (PinObj, roil, roi2, margin, nroi)

Description: add roil and roi2 and expand by margin margin

pinnLib.allBeamsOff (PinObj)

Description: turns display off for all beams

pinnLib.allBeamson (PinObj)

Description: turns all display on for all beams

pinnLib.allRoIsoff (PinObj)

Description: turns display off for all ROIs

pinnLib.applyDVFsToROI ( $P$ inObj, roi, $d v f s$ )

Description: applies dvfs in list $d v f s$ to roi roi and returns ordered list of new names

Arguments: $P i n O b j$ - root pinnacle object

$r o i$ - str - name of roi to be warped

$d v s$ - [] - ordered list of dvfs to apply to roi

pinnLib applyDVFtoROIs (PinObj, mvgImg, fxdImg, $d v f$, rois)

Description: Applies dvf to deform each roi in rois(python list)

Arguments: PinObj - root pinnacle object

mvgImg - moving image name (e.g. PT1_W1_S1)

fxdImg - fixed image name (e.g. PT1_W5_S1)

$d v f$ - str - dvf name (not full path)

rois [] - python list of rois to propagate 
Return: python list containing new roi names.

pinnLib. applyDVFtoVolume (PinObj, mvgImg, $f x d I m g, d v f, d v o l=$ None)

Description: Applies dvf to deform mvgImg

Arguments: PinObj - root pinnacle object

mvgImg - moving image name (e.g. PT1_W1_S1) (must be an exisitng image)

fxdImg - fixed image name (e.g. PT1_W5_S1) (must be an exisitng image)

$d v f$ - name of Pinnacle $9.1 \mathrm{dvf}$ (assumed to be in patient dir)

Return: name of the deformed volume (e.g. PT1_W1_S1 (Demons))

pinnLib . automouseExitPlan (win, save $=$ False)

Description: exits plan by using automouse to click out of plan -requires automouse to be running and assumes original window position has not changed

pinnLib.automouseoff ()

Description: terminates automouse by deleteing file AUTOMOUSEON

pinnLib.automousestart $(x, y)$

Description: starts mouse clicks at screen position $\mathrm{x}, \mathrm{y}$

pinnLib.automousestop ()

Description: suspends mouseclicks by deleteing file AUTOMOUSECMD

pinnLib.autoplacepoI (PinObj, poi, roi)

Description: automatically place point poi at centroid of region roi

pinnLib.calculateDose (PinObj, trial)

Description: calculates dose for trial trial

pinnLib.cleanRoIs (PinObj, rois, min_area=0.2)

Description: cleans each roi in rois

pinnLib. clearDvfBuffer (PinObj, mvgImg, fxdImg, dvfLink, dvfFile)

Description: Clears DVF Buffer for the given dvfLink by renaming dvfLink, creating temp file with same name and using Pinnacle to delte it (which also clears the buffer). Note: A more direct method should be employed in the future.

Arguments: PinObj - root pinnacle object

mvgImg - moving image name

fxdImg - fixed image name

$d v f L i n k$ - symbolic link to dvf file. Must have shortened dvf name following pinnacle conventions.

dvfFile - dvf file associated with

WARNING: This will only clear the buffer if the dvfLink corresponds to the DVF currently loaded into memory

pinnLib.clearObjectives (PinObj)

Description: clears all research objectives

pinnLib.clrRigidReg (PinObj, vol)

Description: clears rigid registration data for volume vol

pinnLib. colorRoIs (PinObj, rois, color)

Description sets color of every roi in rois to color

pinnLib. combineROIs (PinObj, rois, margin, nroi)

Description: add rois in rois and expand by margin margin 
pinnLib.constrainedMinimization (PinObj, trial, $n=100$ )

Description: optimization approach that makes oar objective values equal in an effort to search a broader area of the solution space.

pinnLib.contractROI (PinObj, roi, new_roi, simar, axmar)

Description: expand roi by margin $(\mathrm{cm})$ and store as new_roi

pinnLib.coordTOind (PinObj, img, $x, y, z$ )

Description: returns the nearest voxel indicies of coordinates $x, y, z$, in image img

pinnLib. copyTrial (PinObj, cptrial, trial, img=None)

Description: if trial doesn't already exist, copies trial cptrial and names it trial

pinnLib.createIsodoseLines (PinObj, dose, fxs $=1$ )

Description: creates a standard set of absolute isodose lines at 98,95,80,60,40, and $20 \%$ of dose dose

pinnLib.delAllButFirstPOI ()

Description: deletes all POIs exept first in list

pinnLib.delpoI (PinObj, poi)

Description: removes poi poi

pinnLib.delTrials (PinObj, trials)

Description: deletes trials in list trials

Arguments: PinObj - root pinancle object

trials - list - list of pinnacle trials to be deleted

pinnLib.deleteroIs (PinObj, rois)

Description: deleter all rois in rois

pinnLib.displayRoIs (PinObj, rois, value='Contour')

Description: sets display to contour for all rois in rois[]

pinnLib.displayRoIsOnAll (PinObj, state)

Description: set display of ROIs on image sets that the ROI does not reside

pinnLib.doIMRT (PinObj, trial, max_itr)

Description: initiates IMRT optimization for trial trial with number of iterations set to max_itr optimization type is set to DMPO with max number of segments $=50$

pinnLib.doseColorWashon (PinObj)

Description turns dose color wash display on

pinnLib.doseoff (PinObj)

Description turns dose display off

pinnLib.dvfExists (PinObj, $d v f)$

Description: returns True if dvf name exists in patient directory else returns false

pinnLib.exePinnCommand (PinObj, COMMAND)

Description: Writes COMMAND to a temporary .Script file in plan dir and executes it. Useful when having trouble with python methods.

Arguments: $\mathrm{PinObj}$ - root pinnacle object

COMMAND - str - string to be executed (e.g. Echo = "Hello World"; )

pinnLib.exePinnScript (PinObj, SCRIPT)

Description: Executes traditional pinnacle script. 
Arguments: $P i n O b j$ - root pinnacle object

SCRIPT - full path of .Script file to execute

pinnLib.exitPlan (PinObj, save $=$ False $)$

Description: exits out of plan

pinnLib.expandROI (PinObj, roi, new_roi, simar, axmar)

Description: expand roi by margin $(\mathrm{cm})$ and store as new_roi

pinnLib .genDemonsDVF (PinObj, mvgImg, fxdImg, RIGID=False, TAG=None)

Description: Initiates Pinnacle Demons registration and appends tag $T A G$ if specified

Arguments: PinObj - root pinnacle object

mvgImg - moving image

fxdImg - fixed image

RIGID - rigid registration flag

$T A G$ - tag to be appended to DVF name (e.g. .PINN)

pinnLib.genDemonsDVFs (PinObj, mvgImgs, fxdImgs, RIGID=False, TAG=None)

Description: Registers multiple image volumes using Pinnacle Demons Algorithm. fxdImgs and mvgImgs can be lists of volumes or a single volume. If fxdImgs is a single volume and mvgImgs is a list (or visa versa) all volumes in the list are registered to the single volume. If both are lists they must be of the same size and volumes of the same index are registered

Arguments: PinObj - root pinnacle object

mvgImgs - moving image or list of moving images

fxdImgs - fixed image or list of fixed images

$T A G$ - tag to be appended to dvf name (e.g. .PINN)

Return: Returns a list of names for the DVFs generated

pinnLib.genPCADVFs (PinObj)

Description: generates a set of dvfs to generate PCA model (Registers all PP images to primary)

pinnLib.getDVH (PinObj, trial, roi)

Description: returns x,y DVH values for trial trial and ROI roi and DVH stats min dose, max dose, mean dose, std dev dose

pinnLib.getDemonsDvfName (PinObj, mvgImg, fxdImg, $m v g F s n=N o n e, f x d F s n=N o n e)$

Description: Returns the DVF file name according to Pinnacle conventions

Arguments: PinObj - root pinnacle object

mvgImg - str - moving image

fxdImg - str - fixed image

Note: Assumes first object in VolumeList is primary image. Pinnacle naming scheme incorporates a two digit tag in the name specifying whether the fixed and moving images are primary or fusion volumes.

pinnLib.getFxdFromDVF (PinObj, $d v f)$

Description: returns fixed image name from dvf named according to pinnacle conventions

pinnLib.getMvgFromDVF (PinObj, $d v f)$

Description: returns moving image name from dvf named according to pinnacle conventions 
pinnLib.getNumberOfObjectives (PinObj)

Description: returns number of objectives for currently selected trial.

pinnLib.getobjective (PinObj, $i)$

Description: returns the pinnacle objective object with index $i$

pinnLib.getobjectiveDose $($ PinObj, $i)$

Description: gets dose setting for objective $i$

pinnLib.getobjectiveNames (PinObj)

Description: returns a list of objective names for the currently selected temporal trial

pinnLib.getobjectivePoint (PinObj, $i$ )

Description: return $\mathrm{x} y$ coordinates and color of objective for plotting

pinnLib.getobjectiveROI (PinObj, $i$ )

Description: get obejctive roi name for objective with index $i$

Arguments: $i$ - int - index of objective to modify

pinnLib.getObjectiveType $($ PinObj, $i)$

Description: returns the pinnacle objective object with index $i$

pinnLib.getobjectiveVol (PinObj, $i)$

Description: gets objective volume setting

pinnLib.getobjectiveWeight (PinObj, $i)$

Description: returns objective weight for index $i$

pinnLib.getPatientDir (PinObj)

Description: returns the path of patient dir on disk

Warning: utilizes current working directory to build plan path. Use caution in the context of scripts that manipulate working directory

pinnLib.getPatientNum $($ PinObj)

Description: returns the patient number as contained in the patient directory

pinnLib.getPlanDir (PinObj)

Description: returns the path of the current plan directory

Warning: utilizes current working directory to build plan path. Use caution in the context of scripts that manipulate working directory

pinnLib.getPlanName (PinObj)

Description: returns current plan name

pinnLib.getRoIDice (PinObj, roil, roi2)

Description: Calculates a dice coefficient (range 0-1) for roil and roi2 by calculating $2 *$ the volume of the intersection ROI / (roil volume + roi2 volume)

Arguments: PinObj - root pinnacle object

roil - str - name of first roi

roi 2 - str - name of second roi

pinnLib.getROIcenter (PinObj, roi)

Description: retruns center coordinates of region roi

Arguments: roi - str - name of roi 
pinnLib.getRiskVolsparing (PinObj, rroi, troil, troi2)

Description: calculates difference in risk structure volume included in two targets

Arguments: $\mathrm{PinObj}$ - root pinnacle object

rroi - str - risk structure

troil - str - name of first roi

troi2 - str - name of second roi

pinnLib.getRoiDoseStdDevs (self, roilist)

Description: returns the dose std dev for the ROIs in roilist

Note: roi list should be sorted in the correct order

pinnLib.getRoimass (PinObj, roi)

Description: returns mass of roi roi - no partial volumes are accounted for at edge voxels

pinnLib.getRoiMaxDoses (self, roilist)

Description: returns the max dose for the ROIs in roilist

Note: roi list should be sorted in the correct order

pinnLib.getRoiMeanDoses (self, roilist)

Description: returns the mean dose for the ROIs in roilist

Note: roi list should be sorted in the correct order

pinnLib.getRoiMinDoses (self, roilist)

Description: returns the min dose for the ROIs in roilist

Note: roi list should be sorted in the correct order

pinnLib.getRoiVolumes (PinObj, roilist)

Description: returns the volumes for the ROIs in roilist Note: roi list should be sorted in the preferred order of returned values

pinnLib.getTemporalIndices (PinObj)

Description: returns ordered list of images in temporal object list

pinnLib.getTrialTemporalIndex (PinObj, trial)

Description: returns index in TemporalObjectList of trial trial

pinnLib.getVolumesWithTag (PinObj, TAG, DETAG=None)

Description: Return a list of volumes that conform to search criteria. Volumes containing the substring TAG will be included in the list but will be excluded if they also contain the substring DETAG

Arguments: $T A G$ - search string

DETAG - search string (volumes will be excluded from list)

pinnLib.interp2d $\left(x, y, x \_e v a l\right)$

Description: returns y value corresponding to intermediate value $x \_$eval where $x$ and $y$ are python lists represents discrete ordered points of a function.

pinnLib.intersectROI (PinObj, rois, nroi)

Description: creates roi nroi representing the intersection of all rois in rois (excludes rois with 0 contours)

pinnLib. isRoiContained (PinObj, roil, roi2, delta $=1$ )

Description: returns True if roi2 is contained wholly or partially in roi1 else returns False 


\section{Arguments:}

delta - float - fractional tolerance i.e delta $=1$ returns true if $100 \%$ of volume is contained, delta $=$ .85 returns true if $\% 85$ is contained in volume

pinnLib.isRoiExcluded ( inObj, roil, roi2, delta=0.05)

Description: returns True if roi1 and roi2 are mutually exclusive exclusivity is determined by taking the intersection of the two ROIs; if fractional volume of the resulting ROI < delta rois are assumed to be exclusive

pinnLib. loadBeams (PinObj, tfile, trial)

Description: loads all beams (name, angle, energy) from plan.trial file tfile for trial trial assumed to be stored in tfile

Arguments: tfile - str - full path of plan.trial file

trial - str - name of trial

pinnLib. loadTrial (PinObj, path, trial)

Description: load trial from disk

Arguments: path - str - full path of file to load

trial - str - name of trial to load

pinnLib.match (alist, $s$ )

Description: similar to index for lists except searches for substring among entries i.e. doesn't need to be exact match

pinnLib.msgReassignROI ()

Description: uses pymouse to click "Reassign ROI" button (screen coordinates x:1600,y:680) when moving an ROI to a different image volume

class pinnLib. multViewWindow (PinObj, row =1, col=1, width=250, height=250, xpos=100, ypos=100)

close ()

Description: closes view windows

setorientation (win, orientation)

Description: Sets the volume orienation (axial,sagital,coronal) for view window vwName

Arguments: win - window number

orientation - string specifying orientation. Valid expressions: 'axial','sagital', or 'coronal'.

setSlice (win, slicenum)

Description: sets slice for view window win

Arguments: win - window number (1-3)

slicenum - slice number

setSliceROI (win, roi)

Description: sets the slice of window win to a number containing roi roi

setTrialAll (trial)

Description: sets trial for all view windows to trial

setVolume (win, vol)

Description: Sets the volume to volume volName

Arguments: volName - name of volume to display 
setVolumeAll ( $\mathrm{vol}$ )

Description: sets volume displayed to vol for all windows

pinnLib. normalizeOARObjectiveValues (PinObj, oar)

Description: normalizes weights of all objectives for organ organ

pinnLib.normalizeobjValues (PinObj, norm_value $=0.001$ )

Description: sets all weights such that the value for each objective is = to norm_value

pinnLib. opendB (PinObj)

Description: LoadPatientList = “”; IF .\#”!PatientReviewMode” .THEN .WindowList .LPPatientSelect .Create .ELSE .WindowList .LPPatientSelect .CreateReadOnly = "”; LoadPlanList = "”; InstitutionList .Current .EndMessageBatch = "”"; EndMessageBatchAdd = "EndBatchModePatient"; EndMessageBatchAdd = "EndBatchModePlan"; EndMessageBatchAdd = "EndBatchModeImage";

WindowList.LPPatientSelect.Unrealize = “"”; WindowList.LPPatientSelect.Create = “"”;

pinnLib. openJaws (PinObj, trial, value)

Description: sets jaw positions to value for all beams in trial trial; used to open jaws before IMRT optimization pinnLib. openPlan (PinObj, pat, plan)

Description: launchpad menu script opens patient plan plan (str) for patient pat (int)

pinnLib. outterRingROI (PinObj, roi, newroi, im, om)

Description: creates a ring roi with innermargin padding im and outter margin padding om

pinnLib.pinnAnswerInit (PinObj)

Description: writes path of current transcript file to " /.pinnAnswerInit.txt"

pinnLib.pinnMsg (PinObj, $m s g$ )

Description: prints string $m s g$ to terminal window

pinnLib poicentroidSlice (PinObj, poi)

Description: returns axial, coronal, and and sagital slice numbers (int that order) associated with poi poi

pinnLib.poiposition (PinObj, poi)

Description: returns coordinates of poi poi

pinnLib. radialdist (PinObj, roi, $d v$ )

Description: returns the change in radius for change in vol $d v$ assuming roi roi is a sphere or cylinder (depending on ROI)

pinnLib. reassignRoI ( $\mathrm{PinObj}$, roi, img)

Description: reassigns ROI roi to image $i m g$

pinnLib. removeBeams ( inObj, trial)

Description: removes all beams associated with trial trial

pinnLib . removedVFsymlinks (PinObj)

Description: removes links to dvfs smybolic links

pinnLib. removeIsodoselines ( $P i n O b j)$

Description: removes all isodose lines

pinnLib. renameRoIs (PinObj, oldRois, newRois)

Description: Renames entries in oldRois [] to entries in newRois [] that have the same list index.

Arguments: oldRois[] - python list of ROI names to change

newRois[] - python list of new ROI names

Notes: Corresponding ROI's are assumed to have the same index in their respective lists.

pinnLib. renameVolume (PinObj, oldName, newName) 
Description: Renames Pinnacle volume from oldName to newName

Returns: True: if successful

False: if unsuccessful

pinnLib reoptimizeMinimization (PinObj, trial, $n=100$ )

Description: part of a constrained opitimization approach, OAR objectives are normalized and an optimization is run. Weights are "smashed" and another optimization is run to bring target coverage back up.

pinnLib.reportObjectivesToFile (PinObj, trial, fname)

Description: writes objective settings for trial trial to a csv file with name fname

pinnLib. resetBeams ( inObj, trial=None)

Description: resets IMRT beam aperatures

pinnLib.residualVolume (PinObj, bnd_rois, rois)

Description: constructs a bounding region defined by the range of variation demonstrated by rois in bnd_rois. returns residual volume of rois in rois lying outside the bounding region in the form of a dictionary where the key is name of the roi and the value is volume.

pinnLib.rgdRegToTransFile (PinObj, vol, pat, wk, nimg1, nimg2)

Description: writes a file containing the $\mathrm{x}, \mathrm{y}, \mathrm{z}$ translations and rotations

Arguments: vol-str-secondary vol name

pat - int - patient number

$w k$ - int - week number

nimg 1 - int - primary imgset number

nimg2 - int - secondary imgset number

pinnLib.ringRoI ( PinObj, roi, newroi, margin=1)

Description: creates a ring expansion named newroi of roi roi with margin margin

pinnLib.roiMaskImg (PinObj, roi, imgset)

Description: intended to be used with external contour. expands contour roi by $1 \mathrm{~cm}$ creates binary mask and masks image specified by imgset. saves masked image to <origfilename>.masked

Note: mask is currently saved in dimensions of primary image which will cause function to fail if all images are not of the same size

pinnLib.roiSearch (PinObj, query)

Description: return an ordered list of rois that match a search query query (supports unix style wild cards e.g GTV*)

pinnLib.roisExist (PinObj, rois)

Description: returns True if all rois in rois exist in pinnacle else returns False

pinnLib.roisonAllimgs (PinObj, state)

Description: sets display of ROIs on all images

pinnLib.saveTrial (PinObj, path, trial)

Description: save trial trial to patb

pinnLib. setAccumTarget Img (PinObj, tgt)

Description: sets the target accumulation img to $\operatorname{tg} t$

pinnLib setBeamAngle (PinObj, beamid, angle)

Description: sets beam angle to angle for beam beamid 
Arguments: beamid - int or str - int: index of beam; str: name of beam

angle - int - beam angle in degrees (0-360)

pinnLib.setBeamIsocenter (PinObj, beamid, iso)

Description: sets beam angle to angle for beam beamid

Arguments: beamid - int or str - int: index of beam; str: name of beam

iso - str - name of poi to set as isocenter

pinnLib. setDVFsymlinks (PinObj)

Description: generates symbolic links for all dvf files with additional suffix (e.g. dvf.PINN) in patient directory

NOTE:REMOVES ALL FILES ENDING IN ”.dvf” before creating symlinks

pinnLib. setDVHdisplay (PinObj, trial, rois=None)

Description: returns $\mathrm{x}, \mathrm{y}$ DVH values for trial trial and ROI roi

pinnLib. setDoseGrid (PinObj, rois, res, margin=1)

Description: sets dose grid to include rois

pinnLib.setDoseGridROIs (PinObj, rois)

Description: set dose grid to encompass rois

Arguments: rois - list - python list containing name of rois

pinnLib. setMinOARObjective (PinObj, oar, dose $=100$, volume $=5$ )

Description: sets the volume setting to volume and dose to dose for all objectives pertaining to OAR oar

pinnLib. setobjectiveA (PinObj, $i, a)$

Description: sets EUD "a" value to $a$ for objective $i$

Arguments: $i$ - int - index of objective to modify

$a$ - float - objective weight (typically between $0-1$ or $0-100$ )

pinnLib. setobjectiveConstrain (PinObj, $i$, constrain)

Description: sets objective constraint with index $i$ to contstrain (bool)

Arguments: $i$ - int - index of objective to modify

constrain - bool - constrain state

pinnLib. setobjectiveDose (PinObj, $i$, dose)

Description: set objective dose value for objective $i$

Arguments: $i$ - int - index of objective to modify

dose - float - target dose in cGy

pinnLib.setobjectiveror (PinObj, $i, r o i)$

Description: set obejctive roi to roi for objective with index $i$

Arguments: $i$ - int - index of objective to modify

roi - str - roi name

pinnLib.setObjectiveType (PinObj, $\left.i, o \_t y p e\right)$

Description: set obejctive type (Min Dose, Max Dose, Uniform Dose, Min DVH, Max DVH, Target EUD, Min EUD, Max EUD, ...) to $o \_t y p e$ for objective with index $i$ 
Arguments: $i$ - int - index of objective to modify

o_type - str - objective type

pinnLib. setobjectiveVol ( $P$ inObj, $i$, vol)

Description: set objectove volume value to vol for objective $i$

Arguments: $i$ - int - index of objective to modify

vol - float - percent volume (should range between 0 and 100)

pinnLib. setobjectiveWeight (PinObj, $i$, weight)

Description: set objective $i$ weight to weight

Arguments: $i$ - int - index of objective to modify

weight - float - objective weight (typically between $0-1$ or $0-100$ )

pinnLib.setPlanningobjectives (PinObj, roi_suffix, prescription=6000)

Description: sets standard planning objectives

pinnLib. setROIimg ( inObj, roi, img)

Description: reassigns ROI roi to image img using automouse to automatically respond to pinnacle confiramtion message

pinnLib. setRoiColorScheme (PinObj)

Description: sets colors of major roi groups

pinnLib. setTemporalIndex (PinObj, $i$ )

Description: sets the temporal selection to index $i$

Arguments: $\mathrm{PinObj}$ - root pinnacle object

$i$ - int - temporal index

pinnLib. setTrial (PinObj, trial)

Description: sets current temporal index and trial

pinnLib.setTxTrials (PinObj, trials)

Description: sets trials to use in accumulation of dose and sets the weighting for each assuming a single fraction prescription (188.6 cGy) and 35 fractions (total prescription $6600 \mathrm{cGy}$ )

Arguments: PinObj - root pinnacle object

trials - list - python list of trials to be included for accumulation

pinnLib.smashWeights (PinObj)

Description: sets PTV weight to 100 and all other weights to orders of magnitude smaller

pinnLib. snapshot (PinObj, fname, img=None, trial=None, plane = 'axial', slc=50)

Description: saves .png file snapshot of trial trial, orientation plane, and slice slc to file fname

Arguments: trial - str - trial name

plane - str - axial, coronal, or sagittal

slc - int - slice number

fname - str - full path of file to be saved

pinnLib. sortDVFs $(d v f s)$

Description: sorts dvfs numerically assuming that the number is preceded by 'FX' and followed by ' _' pinnLib.subtractROI (PinObj, roil, roi2, nroi) 
Description: subtract roi2 from roil to create nroi

Arguments: roil - str - name of roi1

roi2 - str - name of roi2

nroi - str - name of new roi

pinnLib.surfdist (PinObj, roil, roi2)

"'Description:** writes vtk meshes to disk calls $\mathrm{C}$ implementation of surfdist and returns a list of distances(mm) from mesh1 vertices to the closest vertice in mesh2

pinnLib.trialImage (PinObj, trial)

Description: returns image name associated with trial trial

pinnLib.trialsearch (PinObj, query)

Description: return an ordered list of trials that match a search query query (supports unix style wild cards)

pinnLib.unionRoI (PinObj, rois, nroi)

Description: creates roi nroi representing the union of all rois in rois

pinnLib.volsearch (PinObj, query)

Description: return an ordered list of volumes that match a search query query (supports unix style wild cards) 


\section{THB_DATABASE MODULE}

class thb_database.TxHsBrDB

Description: main database object

DmetricFROMdb (tx, grp, metric)

Description: returns temporal dose volume metric from database e.g. D95 for group grp and treatment $t x$

DmetricTOdb (tx, grp, metric, UPDT=False)

Description:

Arguments: $t x$ - str - name of treatment

grp - str - name of roi group

VmetricFROMdb (tx, grp, metric)

Description: returns temporal volume metric from database e.g. V95 for group grp and treatment $t x$

VmetricTOdb (tx, grp, metric, UPDT=False)

Description: commits volume metric to database e.g. V20

Arguments: $t x$ - str - name of treatment

grp - str - name of roi group

add_roigroup ( grp, rois, color='red')

Description: adds and roi group named grp containing ROIs rois and sets color to color

add_tx (name, nfxs)

Description: adds treatment to database with name name and number of fractions $n f x s$

all_rois ()

Description: return all rois in roigrps

centroidFROMdb ( $g r p)$

Description: returns centroid magnitude deviations from $\mathrm{db}$

centroidTodb (grp)

Description: calculates centroid magnitude displacement from primary image as a function of fraction and stores it in database. grp should be the base roi name e.g. PTV for PTV_1_1, PTV_PCAAFX2, etc.

clearData ()

Description: clears database signal data ie.e self.signals and self.signalhst

copy_tx (orig, copy)

Description: copies treatment orig to new name copy 
Arguments: orig - str - name of tx to copy

copy - str - new name of copied tx

dateTOfx (tx, date)

Description: returns date associated with fraction $f x$

dbpath ( )

Description: Setup datbase path in patient plan diretory. If path exists return a list of session files (.thb)

del_tx (name)

Description: removes treamtent name from database

doseStatsFROMdb (tx, grp, stat)

Description: extract dose min stats for treatment $t x$ and roi group grp

Arguments: $t x$ - str - name of treatment

grp - str - name of roi group

stat - str - min,max,mean,std; specifies stat to retrieve

doseStatsTOdb (tx, grp, UPDT=False)

Description: commits dose stats (min max mean std) to db for treatment $t x$ and group grp

fXDVHFROMdb $(t x, g r p, f x)$

Description: extracts DVH for a ROI in ROI group grp which corresponds to fraction $f x$ in treatment $t x$ (Normalized volume, absolute dose (cGy))

Arguments: $t x$ - str - name of treatment

grp - str - name of roi group

$f x$ - int - number of fraction

fxDVHSIGvalid ( $t x, g r p, f x)$

Description: returns True if signal exists in self.signals dictionary returns False if incomplete

fxDVHTOdb $(t x, g r p, f x)$

Description: extracts DVH for a ROI in ROI group grp which corresponds to fraction $f x$ in treatment $t x$

Arguments: $t x$-str - name of treatment

grp - str - name of roi group

$f x$ - int - number of fraction

fxDmetric (tx, fx, grp, metric)

Description:

Arguments: $f x$ - int - treatment fraction

grp - str - name of roi group

metric - float - dose metric to calculate i.e. 95 -> D95

fxVmetric (tx, fx, grp, metric)

Description:

Arguments: $f x$ - int - treatment fraction

grp - str - name of roi group

metric - float - dose metric to calculate i.e. 95 -> D95 
imgs INtx $(t x)$

Description: returns a list of images in treatment $t x$

$\operatorname{load}()$

Description: loads saved objects from file into current database

massFROMdb $(t x$, grp $)$

Description: returns $\mathrm{x}, \mathrm{y}$ lists for plotting where $\mathrm{x}$ is the fx number and $\mathrm{y}$ is mass

massSIGvalid ( grp)

Description: returns True if signal exists in self.signals dictionary returns False if incomplete

massTOdb (tx, grp, UPDT=False)

Description:

Arguments: $t x$ - str - name of treatment

grp - str - name of roi group

rename_tx (oldname, newname)

Description: renames treatment object

Notes: use with care - new object is set to old object with new name and old object is deleted.

roiTOsigkey (tx, roi)

Description: return a list of signal key tuples ( format: tuple(roi,trial) ) for querying signal dicitionary self.signal \{\}

Arguments: $t x$ - str - name of treatment

roi - str - name of roi

roiTotrials $(t x, r o i)$

Description: return a dict of fxs and associated trials corresponding to roi roi

roisINtx $(t x)$

Description: returns that correspond to treatment $t x$

Arguments: $t x$ - str - name of treatment

save ()

Description: save db to pickle file with name self.name (with whitespace and other characters stripped to make a suitable file name.

savedata $($ fname $=$ None $)$

Description: save data in format that can be accessed outside of Pinnacle (using simple data structures)

set_file_name()

Description: sets attribute self.file based on attribute self.name which is the name of database / seesion. all whitespace and special chars(-) are removed and a .thb extension is apppended. Returns True if succesful

trialDVHFROMdb (trial, grp)

Description: returns trial DVH and dose stats from database fro trial trial and group grp

trialDVHTOdb (trial, grp)

Description: commits DVH and dose stats to databse for trial trial

trialDmetric (trial, grp, metric)

Description: returns dose metric from database e.g. D95 for group grp and trial trial

trialdosestat (trial, grp, stat)

Description: returns dose statistic for trial trial and group grp 
Arguments: stat - str - min dose, max dose, mean dose

trialVmetric (trial, grp, metric)

Description: returns volume metric from database e.g. V95 for group grp and trial trial

tx_doses_exist $(t x)$

Description: return True if dose has been calculated for all trials in treatment $t x$

Arguments: $t x$ - str - name of treatment to check

tx_img_offsets $(t x)$

Description: return True if all synth img exist and offsets are 0 else return False

Arguments: $t x-$ str - name of treatment to check

tx_imgs_exist $(t x)$

Description: return True if all images for a given treatment currently exist in Pinnacle

Arguments: $t x$-str - name of treatment to check

tx_trials $(t x)$

Description: returns all trials in treatment tx

tx_trials_exist $(t x)$

Description: return True if all trials for a given treatment currently exist in Pinnacle

Arguments: $t x-$ str - name of treatment to check

updt_sig_rois ()

Description: initialize subdicionaries for all rois in roigrps

volumeFROMdb ( $t x$, grp)

Description: returns $\mathrm{x}, \mathrm{y}$ lists for plotting where $\mathrm{x}$ is the $\mathrm{fx}$ number and $\mathrm{y}$ is volume (cc)

volumeSIGvalid ( $g r p$ )

Description: returns True if signal exists in self.signals dictionary returns False if incomplete

volumeTOdb (tx, grp, UPDT=False $)$

Description: Extracts roi volumes from pinnacle for rois in roigroup grp that correspond to images in treatment $t x$ and stores them in database.

Arguments: $t x$ - str - name of treatment

grp - str - name of roi group

class thb_database.dlist

Bases: dict

Description: python dictionary with ordered list of entries

append (key, value)

Description" append dicionary entry

insert (key, value, ikey)

Description: add new dictionary entry with key key and value value; insert key into self.list before ikey

remove (key)

Description: removes key key from dlist

class thb_database.fxobj

Description: fraction data structure 
Attributes: date(datetime) - date of fraction (datetime.date object)

$\operatorname{img}(\mathrm{str})$ - associated image name

trial(str) - associated trial name

thb_database.gen_fx_schedule (nfxs, start)

Description: Generates a list of datetime objects corresponding to a fractionation schedule containing $n f x s$ and beginning on date start. Fractions are scheduled sequentially on weekdays.

Arguments: $n f x s$ - integer - number of fractions in treatment

start - datetime object - date associated with first fraction

Returns: fxdates - [] - list of datetime objects

thb_database.interpx $\left(x, y, x \_\right.$eval $)$

Description: interpolates function specified by $x$ and $y$ python lists at $x \_$eval

thb_database.interpy $\left(y, x, y \_e v a l\right)$

Description: interpolates function specified by $x$ and $y$ python lists at $y \_$eval

class thb_database.roigroup

Bases: list

Description: python list containing roi names for a given roi group. Additional attributes include color and display settings for the group

clear ()

Description: clears all elements in list

update (rois)

Description: replaces current list with list rois

class thb_database.txobj

Description: fraction data structure

Attributes: name - str - treatment name

$f x-\{\}-$ dict of fxobjs; key $=$ fx num

trial(str) - associated trial name

def_art_sched ()

Description: sets member attribute self.sched which is a dictionary of common adaptive schedules assuming 35 fractions

dt_to_fx (date)

Description: return fx number for a given date (assumes no more than $1 \mathrm{fx}$ per day)

roisInTx ()

Description: returns a list of all rois corresponding to fractions in tx

updtFxRoilsts (PinObj)

Description: updates roi lists for each $\mathrm{fx}$ 


\section{THB_MAINWINDOW MODULE}

class thb_mainwindow. Mainwindow (PinObj, $T x H s B r D B$, txname $=$ None, parent $=$ None $)$

Description: Treatment History Browser main window form (subclass of QMainWindo)

Brws_quit_QPushButton_clicked ()

Description: closes main window

Brws_save_QPushButton_clicked ()

Description: saves session file

Brws_save_and_quit_QPushButton_clicked ()

Description: saves session file and exits

Data_blue_QPushButton_clicked ()

Description: generates snapshots and tx report DVHs for adaptive planning studies

Data_committ_all_data_to_db()

Description: committs all data to the database and saves the database DVH based data are not explicitly commited because they can be derived from data committed via fxDVHTOdb()

Data_gray_QPushButton_clicked ()

Description: generates all synthetic images and rois for currently selected treatment (self.tx)

Data_green_QPushButton_clicked ( )

Description: auto replanning based on primary plan and treatment specification of adaptive fractions

Data_red_QPushButton_clicked ( )

Description: runs all simulation steps - equivelant to pushing grey, green, and blue buttons for each tx

File_Quit_QAction_triggered()

Description: quits without saving database file

File_Save_session_QAction_triggered ( )

Description: saves database file

Fx_QCalendarWidget_selectionChanged ( )

Description: Slot function for selection changed signal from self.ui.Fx_QCalendarWidget. Updates all fraction dependent fields i.e. fraction indicators, fraction statistics, image volume (if img view bound to fx) etc

Fx_next_QPushButton_clicked ( )

Description: slot: increments current $\mathrm{fx}$

Fx_prev_QPushButton_clicked ()

Description: slot: decrements current fx 
Img_new_QPushButton_clicked ( )

Description: slot - brings up new image viewer

Img_rois_off_QPushButton_clicked ()

Description: turns display of all ROIs off

Plot1_context_menu ()

Description: builds context menu for plot 1

Plot1_open_in_editor ( )

Description: open plot in external editor

Plot1_plot_ePushButton_clicked ()

Description: update plot in plot area one according to current selection

Plot1_update_data ( )

Description: forces data query from pinnacle before updating plot

Plot2_context_menu ()

Description: builds context menu for plot 2

Plot2_open_in_editor( )

Description: open plot in external editor

Plot2_plot_QPushButton_clicked ()

Description: update plot in plot area one according to current selection

Plot2_update_data ()

Description: forces data query from pinnacle before updating plot

ROIGrp_1_QComboBox_currentIndexChanged ( )

Description: slot: called when combo box selection is changed sets labels ...

ROIGrp_1_next_QPushButton_clicked ( )

Description: slot - increments ROIGrp_QComboBox selection

ROIGrp_1_prev_QPushButton_clicked ( )

Description: slot - decrements ROIGrp_QComboBox selection

ROIGrp_2_QComboBox_currentIndexChanged ( )

Description: slot: called when combo box selection is changed sets labels ...

ROIGrp_2_next_QPushButton_clicked ()

Description: slot - increments ROIGrp_QComboBox selection

ROIGrp_2_prev_QPushButton_clicked ()

Description: slot - decrements ROIGrp_QComboBox selection

Scripts_QColumnView_doubleclicked (index)

Description: slot for double click on script - executes selected python script

Signal_1_QComboBox_currentIndexChanged ( )

Description: slot: called when combo box selection is changed sets labels ...

Signal_1_next_QPushButton_clicked ()

Description: slot - increments Signal_QComboBox selection

Signal_1_prev_QPushButton_clicked ( )

Description: slot - decrements Signal_QComboBox selection

Signal_2_QComboBox_currentIndexChanged ( )

Description: slot: called when combo box selection is changed sets labels ... 
Signal_2_next_QPushButton_clicked()

Description: slot - increments Signal_QComboBox selection

Signal_2_prev_QPushButton_clicked()

Description: slot - decrements Signal_QComboBox selection

Tx_1_QComboBox_current IndexChanged ()

Description: slot: called when combo box selection is changed sets labels ...

Tx_1_next_QPushButton_clicked ()

Description: slot - increments Tx_QComboBox selection

Tx_1_prev_QPushButton_clicked ()

Description: slot - decrements Tx_QComboBox selection

Tx_2_QComboBox_current IndexChanged ()

Description: slot: called when combo box selection is changed sets labels ...

Tx_2_next_QPushButton_clicked ()

Description: slot - increments Tx_QComboBox selection

Tx_2_prev_QPushButton_clicked ()

Description: slot - decrements Tx_QComboBox selection

Tx_QComboBox_current IndexChanged ()

Description: slot: called when combo box selection is changed sets labels ...

Tx_next_QPushButton_clicked ()

Description: slot - increments Tx_QComboBox selection

Tx_prev_QPushButton_clicked ()

Description: slot - decrements Tx_QComboBox selection

Util_Python_console_QAction_triggered ()

Description: utilities menu action - bring up console

Util_ROI_group_colors_QAction_triggered ()

Description: utilities menu action - bring up ROI color editor

Util_ROI_group_editor_QAction_triggered ()

Description: utilities menu action - bring up ROI group editor

Util_Treatment_editor_QAction_triggered ()

Description: utilities menu action - bring up treatment editor

add_signal ()

Description: inititates dialogue to enter a new dose / volume metric (e.g. D95 / V20)

clear_msg ( )

Description: clears browser msg

clear_plot1_compare ()

Description: clears all reference data settings (e.g. reference roi groups tx's etc.)

clear_plot2_compare ()

Description: clears all reference data settings for plot 2 (e.g. reference roi groups, tx's etc.)

img_view_beams_ImgCheckBox_clicked ( )

Description: toggles beam display

img_view_dose_ImgCheckBox_clicked ( )

Description: toggles dose colorwash 
img_view_roi_ImgCheckBox_clicked ()

Description: toggles display for all rois in all roi groups

objReport ( )

Description: prints primary trial objectives to file

popRoiGrpBoxes ( )

Descriptions: populates RoiGrp Comboboxes

popSignalBox1 ( )

Descriptions: populates signal box associated with plot 1 - signal lists depend on selected roi group popSignalBox2 ( )

Descriptions: populates signal box associated with plot 2 - signal lists depend on selected roi group popTxBoxes ( )

Description: populates all treatment comobo boxes from database

primaryROIgroup ( )

Description: script to set primary rois

refresh_form_db (txname=None)

Description: db dependent setup. called when new datebase is loaded to reinitiallize GUI.

refresh_form_tx ()

Description: tx specific setup to be invoked anytime tx is changed.

set ImageViewF $\mathbf{x}(t x, f x)$

Description: changes the image and dose volume to those corresponding to treatment $t x$ and fraction $f x$

setPlanningObjectives $(f x)$

Description: sets a standard group of planning objectives for fraction $f x$

set_cal_color (color)

Description: sets color to color for current treatment

set_msg (msg, mtype)

Description: displays browser message $=m s g$ with icon specified by mtype

Arguments: $m s g$ - str - message to display

mtype - str - icon to display: 'warn' or 'info'

set_plot1_compare_dvh_roi_groups ( )

Description: opens dialog for plot 1 to set reference dvh roi groups

set_plot1_compare_roi_groups ()

Description: opens dialog for plot 1 to set reference roi groups

set_plot1_compare_treatments ()

Description: opens dialogue for plot 1 to set reference treatments

set_plot2_compare_dvh_roi_groups ( )

Description: opens dialog for plot 2 to set reference dvh roi groups

set_plot2_compare_roi_groups ()

Description: opens dialog for plot 2 to set reference roi groups

set_plot2_compare_treatments ()

Description: opens dialog for plot 2 to set reference treatments

set_roi_group_signals_from_plot1()

Description: opens dialog for plot 1 to set reference roi group signals 
set_roi_group_signals_from_plot2()

Description: opens dialog for plot 2 to set reference roi group signals

setupGUI ( )

Description: populates form icons and data

sig_to_slots ()

Description: connects GUI signals to GUI class functions (slots)

standardROIgroups ( )

Description: script to set standar ROI groups

txReportDVHs ( )

Description: prints DVHs to file for all standard roi groups and treatments.

txReportImgs (txs)

Description: prints plan snapshot and DVHs to file

Arguments: txs - list - python list of treatment names

updateDataSummary ( )

Description: updates data table under the data tab

updatePlot ( $p$, editor $=$ False, save $=$ None $)$

Description: plots data from from multiple tx's and roigrps for a given signal

Arguments: p - int - plot index (1 or 2)

editor - bool - True brings up plot in external editor

save - str - full path of file to be saved if $=$ None no file is saved

thb_mainwindow.get_signal_label (signal)

Description: returns a string containing the appropriate signal name and unit associated with signal signal 


\section{Appendix II}

Synthetic patient datasets for lung cancer radiotherapy research using principal component analysis. 


\section{Synthetic patient datasets for lung cancer radiotherapy research using principal component analysis.}

Christian Dial", B.Sc., Jeffrey V. Siebers ${ }^{\dagger}$, Ph.D., Geoffrey D. Hugo*, Ph.D.

"Department of Radiation Oncology, Virginia Commonwealth University, Richmond, VA, 23298

'Department of Radiation Oncology, University of Virginia, Charlottesville, VA, 22908

\section{Corresponding author}

Geoffrey D. Hugo, Ph.D.

Department of Radiation Oncology

Virginia Commonwealth University

401 College Street

PO Box 980058

Richmond, VA 23298

Phone: 804-628-3457

Fax: 804-628-0271

E-mail: gdhugo@vcu.edu

\section{Conflicts of interest}

The authors have no actual or potential conflicts of interest to disclose. 


\begin{abstract}
Purpose: Present a method for sampling clinically relevant geometries that demonstrate temporal anatomical trends for use in radiation therapy research.
\end{abstract}

Methods: Weekly images associated with the radiation treatment course of 13 non-small-cell cancer patients are each registered to the first in the series, and resulting displacement vectors are combined into a joint variation matrix. Principal component analysis (PCA) of the matrix is performed resulting in a set of basis vectors and associated coefficients which may be used to reconstruct the original deformations. A linear fit of temporally ordered coefficients is performed and fit-residuals are utilized to construct a patient-specific probability density function. Coefficients that correspond to intermediate geometries are sampled by evaluating the fit and adding a random component from the patient-specific distribution; sampled coefficients are then utilized to generate intermediate deformations corresponding to each fraction of a radiation therapy schedule and used to deform the initial image and associated contours. Synthetic datasets are validated by: 1) comparing regression trends in synthetic images to those reported in the literature for actual datasets, 2) comparing variation of relevant risk-structure contours (lungs, heart, esophagus, and spinal cord) in synthetic images to that observed in homologous structures warped using the original mappings, and 3) comparing the latter with physician drawn contours on the original datasets to demonstrate that they themselves are reasonable. Differences are quantified dosimetrically in terms of cumulative dose-volume metrics, and geometrically in terms of the mean of minimum-surface-distance distributions (mean MSD).

Results: For all risk-structures, $80 \%$ of all mean MSD differences between physician drawn contours and those warped with original mappings fell below $2 \mathrm{~mm}$. For warped and synthetic contours, $95 \%$ of mean MSD differences were less than $2 \mathrm{~mm}$ with $86 \%$ falling below $1 \mathrm{~mm}$. Dosimetric differences between dose calculated on original images and that calculated on synthetic images were less than $5 \%$ for the majority of comparisons (89\%). Average gross tumor volume regression for synthetic datasets was $36.8 \%(11.8-76.4 \%)$, with average daily regression equal to $1.1 \%$; these values are similar to those reported by others for in lung cancer. 
Conclusion: Synthetic datasets that demonstrate relevant temporal trends may be generated by sampling PCA coefficients using a linear fit of time-ordered coefficients and a patient-specific distribution of fit residuals.

\section{Key words}

Principal component analysis, synthetic dataset, tumor regression

\section{Introduction}

Representations of intra- and inter-fraction anatomical variation are necessary in modern radiation therapy for quantifying dosimetric variation, specifying treatment margins, and informing new planning strategies such as adaptive radiation therapy. However, it can be difficult to collect enough images to directly represent anatomical variability for a single patient or a population. One way of simplifying this representation is by generating a low-dimensional statistical model based on a set of images from which synthetic geometries may be sampled. REFS This approach was implemented by Söhn et al who used principal component analysis (PCA) to model inter-fraction variability in prostate cases, assuming an underlying Gaussian distribution, to examine the dosimetric consequences of geometric uncertainty. ${ }^{[1]}$ While this is appropriate in cases where variability is entirely stochastic and stationary, temporal trends in the data are not accurately modeled by this method. In a study conducted by Badawi et al, PCA was used to retrospectively and prospectively reconstruct temporal trending data associated with a cohort of lung cancer patients to within several millimeters when three to four principal components were utilized; ${ }^{[2]}$ however, a method for generating synthetic samples is not addressed.

A useful application of synthetic geometries that demonstrate temporal trends is in planning studies that adapt treatment to observed variation. Periodic re-planning to accommodate regressing tumor volumes may decrease exposure to surrounding tissue or allow for isotoxic dose escalation. ${ }^{[3-6]}$ One limit of such studies is the availability of datasets to characterize the anatomy of the day and 
examine the full potential of adaptive therapy. Furthermore, cumulative measures of dose used to evaluate adaptive schedules are subject to mapping errors associated with image registration.

In this work we implement global statistical modeling of interfraction variability to produce synthetic CT datasets for each fraction of treatment that demonstrate temporal anatomical trends (e.g. tumor regression) and are consistent with an underlying mapping for use in adaptive radiotherapy planning studies.

\section{Methods}

\section{Overview of methods}

Statistical modeling of inter-fraction variability is accomplished using PCA to decompose observed variation into a basis set of transform vectors and a set of weighting coefficients which correspond to each input transform. Resulting coefficients for each basis vector are plotted verses the elapsed time associated with the corresponding input transform and a linear fit is extracted. Coefficients associated with arbitrary time-points are then generated by evaluating the fit at the desired time and sampling from a residual distribution generated using input residuals and kernel density estimation. This method preserves temporal trends in the sampling process and is utilized to generate a set of transforms, images, and contours associated with each fraction of treatment.

\section{Patient data}

Weekly fan beam CT scans, ranging from $4-7$ per patient, are obtained throughout the course of a typical fractionation schedule for 13 non-small-cell lung cancer (NSCLC) patients undergoing definitive radiation therapy treatment. Scans are acquired with a voxel size of $1.2 \times 1.2 \times 2.0 \mathrm{~mm}^{3}$ under active breathing control (ABC) ${ }^{[7]}$ Relevant structures are manually contoured on each weekly dataset by a qualified physician including: gross tumor, nodal involvement, spinal cord, lungs, heart, and esophagus.

For each set of images for each patient, a bony alignment is performed and the first image in the series is registered to all subsequent images using the Demons deformable algorithm as implemented in 
the Pinnacle treatment planning system (Philips Oncology, Fitchburg, WI). For each patient, a common region of interest and resolution are used for weekly registrations resulting in displacement vector fields (DVF) of the same size that contain data for homologous points in corresponding elements.

\section{PCA model of interfraction variation}

Each DVF containing $m$ points is concatenated into a single vector $\boldsymbol{p}(t)$ containing translations in the $x, y$, and $z$ dimensions. The $n$ weekly DVFs for each patient are joined sequentially into a joint variation matrix $\boldsymbol{V}$,

$$
\mathbf{V}=\left[\begin{array}{ccc}
x_{11} & \cdots & x_{1 n} \\
y_{11} & & y_{1 n} \\
z_{11} & & z_{1 n} \\
\vdots & \ddots & \vdots \\
x_{m 1} & & x_{m n} \\
y_{m 1} & & y_{m n} \\
z_{m 1} & \cdots & z_{m n}
\end{array}\right],
$$

such that each row contains data for homologous points that are organized temporally from left to right. The matrix is then mean-corrected by subtracting the row means from each row.

PCA is performed to decompose the joint variation matrix into a mean vector $\bar{p}$ and a set of

orthonormal basis vectors $\left\{\boldsymbol{q}_{1}, \boldsymbol{q}_{2}, \ldots, \boldsymbol{q}_{n}\right\}$. A weighted sum of the resulting basis vectors may be used to reconstruct a DVF associated with a given time-point as follows,

$$
\mathbf{p}(t)=\overline{\mathbf{p}}+\sum_{i=1}^{n} c_{i}(t) \mathbf{q}_{i},
$$

where $c_{i}(t)$ is the principal coefficient associated with basis vector $i$ and time-point $t$ defined as,

$$
c_{i}(t)=(\mathbf{p}(t)-\overline{\mathbf{p}}) \cdot \mathbf{q}_{i}
$$

\section{Synthetic datasets}

For each basis vector resulting from the analysis, associated principal coefficients for all weekly DVFs are plotted as a function of time and a linear fit of the data is performed. Fit-residuals are used to 
construct a patient-specific probability density function $\hat{f}(x)$ via kernel density estimation according to

the following equation:

$$
\hat{f}(x)=\frac{1}{n h} \sum_{i=1}^{n} K\left(\frac{x-x_{i}}{h}\right)
$$

where $x_{i}$ is the $i$ th fit residual, $K$ is the kernel smoothing function, and $h$ is a parameter known as the bandwidth which serves to scale the width of the kernel. In estimating the residual distribution, a Gaussian kernel function is utilized in conjunction with a bandwidth parameter equal to the standard deviation of the fit residuals.

After fitting coefficient data and estimating residual distributions, a set of coefficients associated with time-points corresponding to 35 fractions of a typical treatment regimen are produced by evaluating the linear fit at each time-point and adding a random component sampled from the associated distribution (example given in Fig. 1). Resulting coefficients are used to generate synthetic DVFs according to Eq. (1), and a pseudo-inverse DVF is calculated as described by Yan et al. ${ }^{[8]}$ Pseudoinverse DVFs are used to warp the primary image and contours resulting in a synthetic image, a set of contours, and a DVF that are self consistent for each fraction.

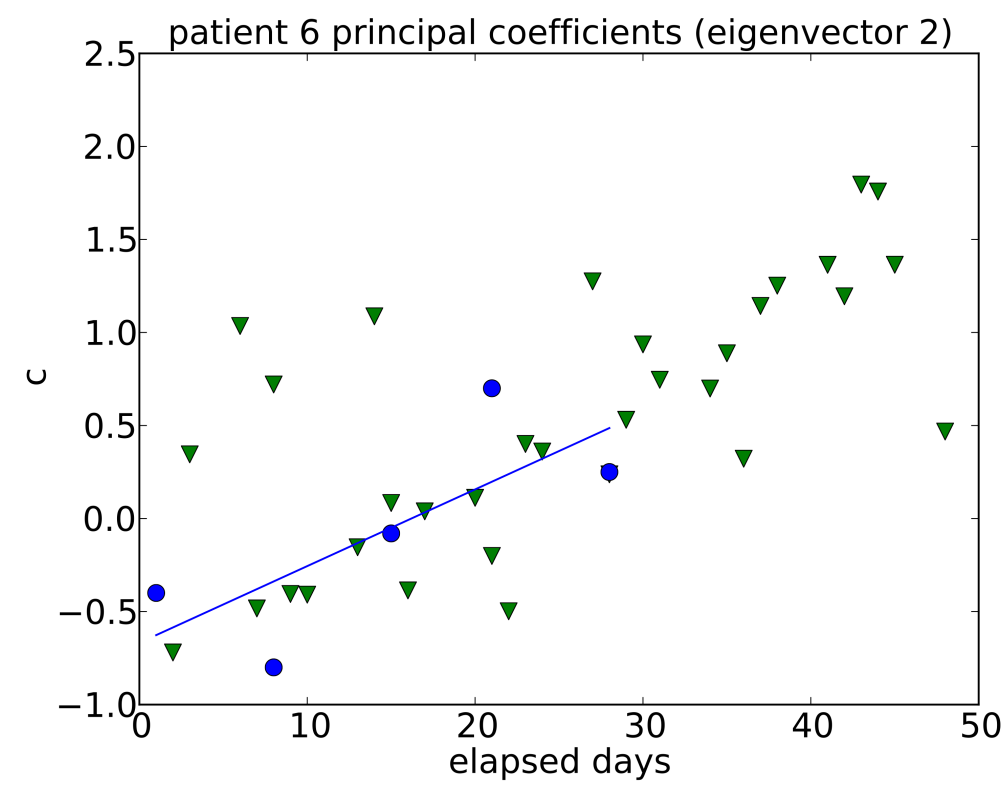

Fig. 1. Patient 6 PCA coefficients associated with 2nd eigenvector. A linear fit of original PCA coefficients (circle marker) is calculated, PCA coefficients associated with intermediate time points are extracted by evaluating the line at the given time-point and sampling a residual from a patient specific PDF constructed using kernel density estimation. 


\section{Validation of synthetic datasets}

In utilizing synthetic datasets in planning studies and other applications it is important that synthetic data represent reasonable poses of patient anatomy, and that time trends be both realistic and clinically relevant. For each risk structure (lungs, heart, esophagus, and spinal cord), deformation over the course of treatment is characterized by calculating minimum surface distances (MSD) between primary and subsequent contours and is used to validate synthetic datasets. For each point $x$ in primary contour $X, \mathrm{MSD}_{\mathrm{x}}$ is defined as

$$
M S D_{x}=\min \{d(x, y) \mid y \in Y\},
$$

where $d(x, y)$ is the Euclidean distance between points $x$ and $y$, and $Y$ is the contour being compared. Each contour comparison results in a distribution of MSDs that are combined to create a composite distribution for each structure and sequence of images. The mean MSD of the composite distribution for each patient and risk structure is then calculated on: 1) manually drawn physician contours on weekly imagesets (manual); 2) warped contours propagated using weekly registrations (warped); and 3) warped contours propagated using synthetic DVFs generated using PCA modeling (synthetic).

Sampled anatomies are expected to vary from observed instances in statistically meaningful ways thus comparisons between individual images may not be used to validate synthetic datasets. The mean MSD is a single metric that is used to characterize deformation over the entire course of treatment and is expected to be similar between synthetic and actual contours for a given risk structure.

Synthetic datasets are validated by evaluating the difference between warped and synthetic mean MSDs. Similarity in the magnitude of deformation over the course of treatment suggests that the synthetic datasets approximate actual anatomy in relevant poses under the assumption that warped datasets are themselves reasonable. The latter is determined by carrying out a similar analysis between manual and warped mean MSDs for each patient and risk structure. This analysis is an evaluation of the quality of the initial registrations upon which statistical sampling is based and utilizes physician contours as a gold-standard. While accuracy of the employed deformation algorithm is not a primary 
objective of this study, mean MSD comparisons are intended to reveal large failures in weekly registrations and to determine if resulting warped contours are reasonable.

In addition to geometric comparisons based on MSDs, dose based metrics between warped and synthetic datasets are reported to facilitate an acceptance criteria by articulating differences in terms of clinically relevant deviations in dose volume histograms (DVH). Intensity modulated radiation therapy (IMRT) plans are developed for each patient on the primary image according to planning criteria specified in RTOG protocol 0839. Dose is calculated on all warped and synthetic images, and is warped back to the primary imageset. Differences between warped and synthetic data in the percent volume of lung receiving at least 20Gy (V20), the percent volume of heart receiving at least 45Gy (V45), the dose received by $2 \%$ of the cord volume (D2), and the mean dose received by the esophagus are reported.

\section{Results}

\section{Manual and warped mean MSD comparison}

Mean MSD differences between manual and warped contours for each patient and risk structure are summarized in Fig. 2. The majority of structures demonstrated similar magnitudes of deformation over the course of treatment, with $80 \%$ of all mean MSD differences falling below $2 \mathrm{~mm}$. The largest deviations were associated with physician contours that resided in low contrast regions with no one structure exhibiting large differences over the entire patient population.

\section{Synthetic and warped mean MSD comparison}

Differences between mean MSDs for warped and synthetic contours are reported in Fig. 3 and demonstrate good agreement in the magnitude of deformation observed; 95\% of mean MSD differences are less than $2 \mathrm{~mm}$ with $86 \%$ falling below $1 \mathrm{~mm}$ for all contours. The two patients exhibiting the largest deviations between warped and synthetic MSDs also demonstrated the largest amount of variation in warped contour geometry resulting in a wider sampling distribution. 


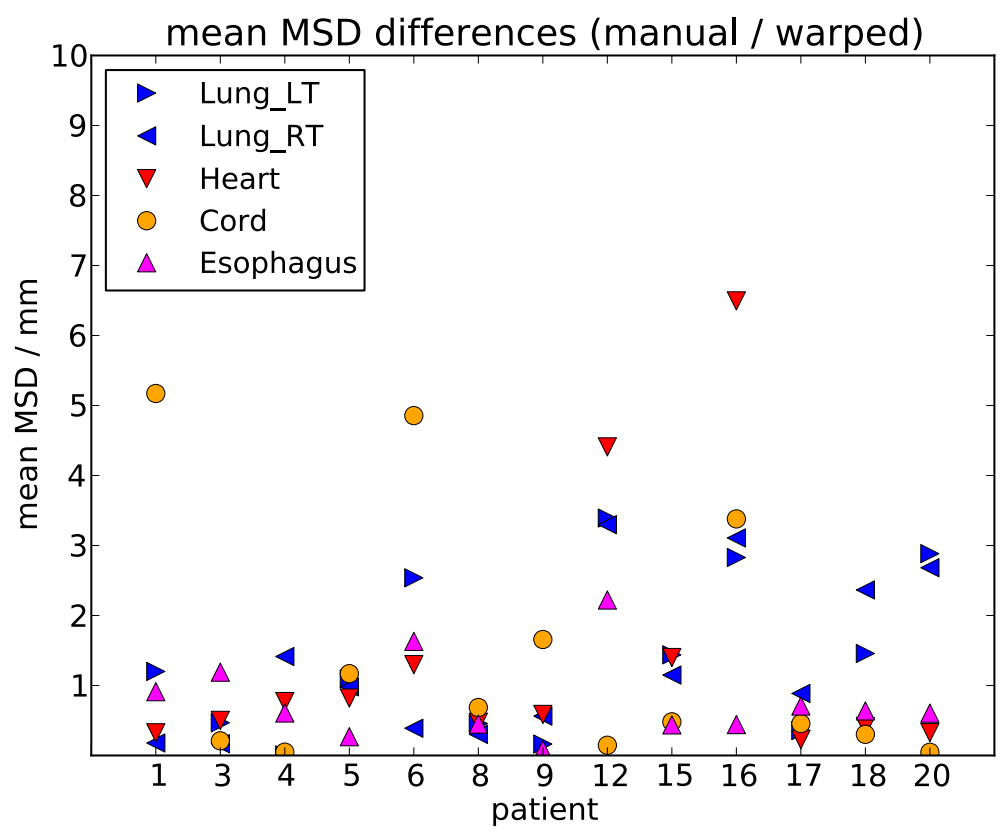

Fig. 2. Mean MSD differences between physician-drawn (manual) contours and those warped with weekly registrations.

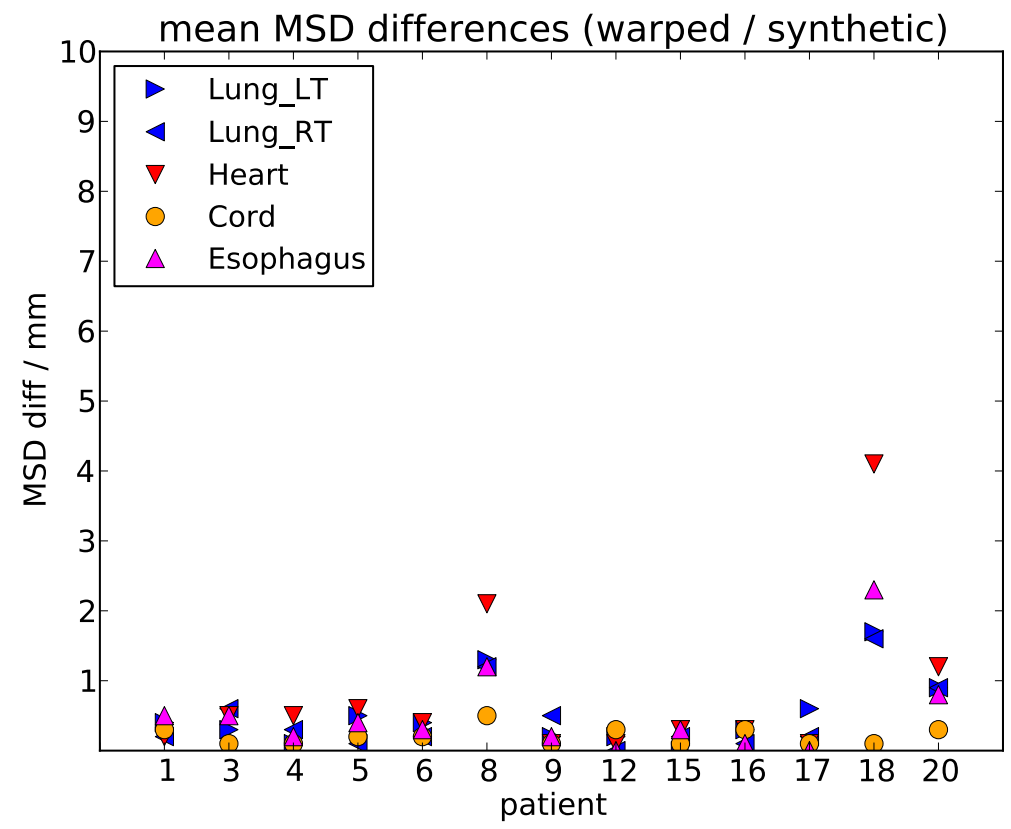

Fig. 3. Mean MSD differences between warped and synthetic contours for each patient and risk structure.

\section{Dose comparisons}

Comparison of cumulative dose metrics between warped and synthetic datasets are summarized in Fig. 4, and DVHs for patients with the best and worst dosimetric agreement are illustrated in Fig. 5. 
$89 \%$ of dose-metric differences were less than $5 \%$ for all structures; the worst agreement over all contours for a single patient was found in the patient with the largest geometric variation.

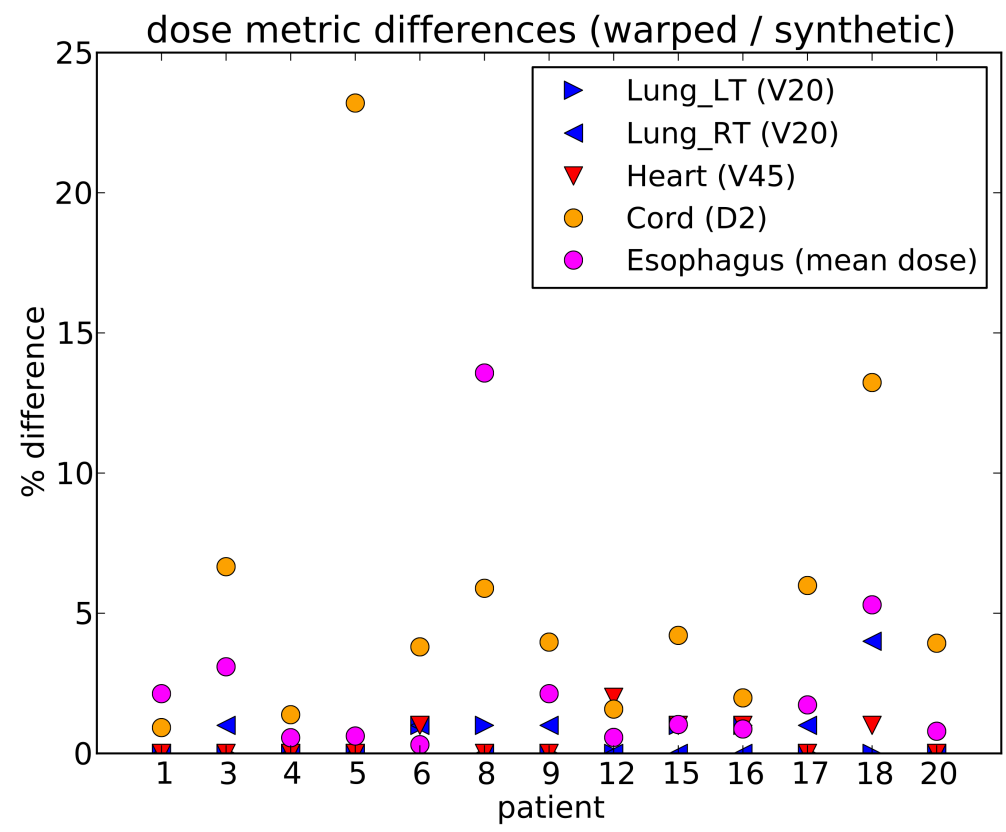

Fig. 4. Percent difference for lung V20, heart V45, cord D2, and esophagus mean dose between warped and synthetic contours.
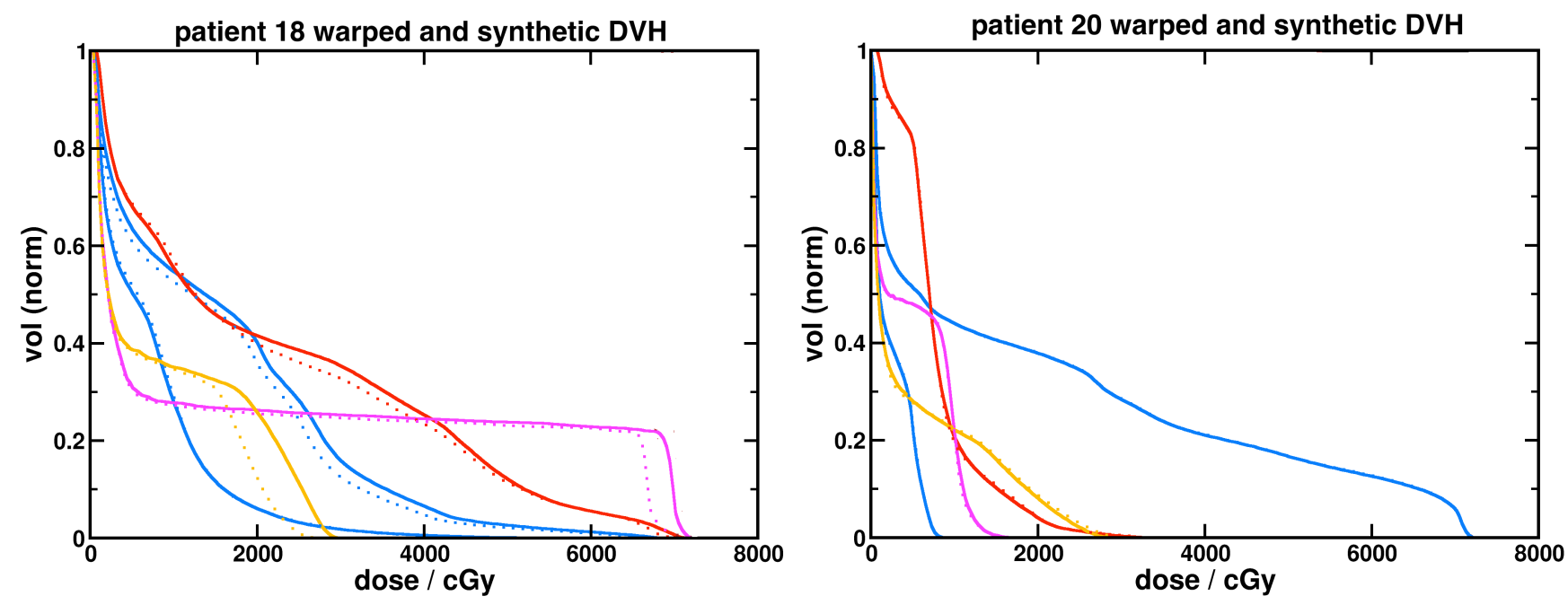

Fig. 5. Dose volume histograms associated with warped (solid) and synthetic (dotted) risk structures for patient 18 (left - demonstrated worst agreement) and patient 20 (right - demonstrated best agreement). Plotted structures include: ipsa- and contra-lateral lung (blue), heart (red), cord (orange), and esophagus (purple).

\section{Volume regression}

Percent of original gross tumor volume as a function of treatment fraction is reported in Fig. 6.

The mean decrease over the course of treatment for all patients was 36.8\% (11.8-76.4\%), and average daily regression was $1.1 \%$. These values are consistent with those reported by others for lung (Table 1). 


\begin{tabular}{lll}
\multicolumn{3}{c}{ GROSS TUMOR VOLUME REGRESSION } \\
\hline \hline author & $\begin{array}{l}\text { \% decrease } \\
\text { in GTV }\end{array}$ & range \\
\hline Woodford et. al. & $38 \%$ & $12-87 \%$ \\
Kupelian et. al. & $42 \%$ & $8-71 \%$ \\
van Zwienen et. al. & $37 \%$ & - \\
\hline \hline
\end{tabular}

Table 1. Average percent decrease in GTV volumes as reported in the literature.

Absolute gross tumor volume as measured on the primary image had a median value of $53.2 \mathrm{~cm}^{3}$ for all patients and ranged between $0.6-377.3 \mathrm{~cm}^{3}$, with the smallest absolute tumor volume demonstrating the largest percent variation over treatment.

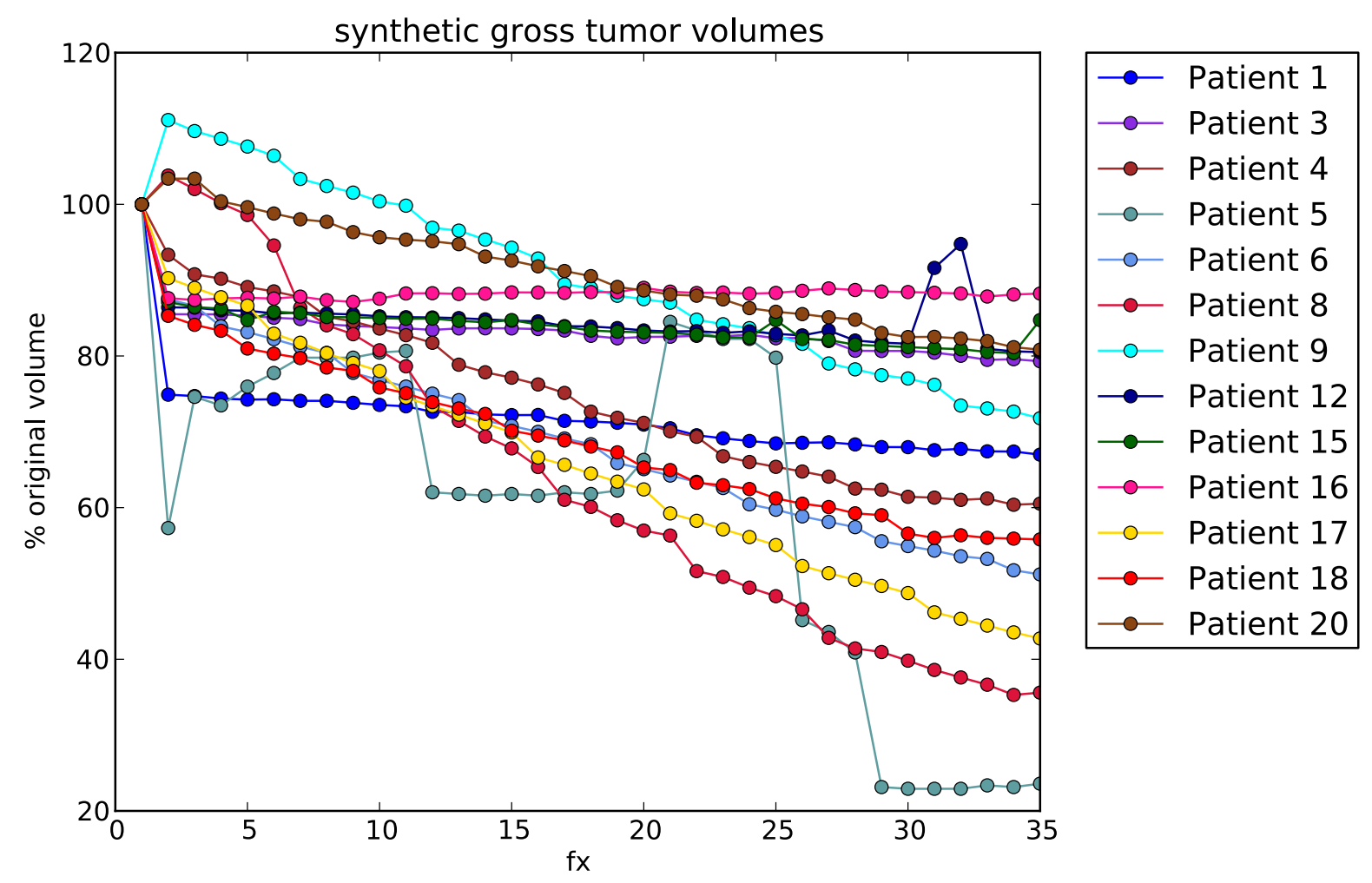

Fig. 6. Percent gross tumor volumes as a function of treatment fraction for synthetic datasets.

\section{Discussion}

To the knowledge of the authors, using a linear fit of time-ordered PCA coefficients to sample synthetic DVFs from a patient-specific PDF of fit residuals represents a novel method of generating clinically relevant anatomies that demonstrate temporal trends. Using this method, a set of self- 
consistent contours, images, and mappings to the primary image, are generated for each fraction in a typical treatment regimen and are intended to be utilized in virtual trials of adaptive radiotherapy.

Clinical relevance was validated by comparing synthetic contours to warped weekly contours, and warped weekly contours to weekly physician contours, to determine if synthetic data represents reasonable poses of patient anatomy. Mean MSDs of a composite distribution for each structure, made up of MSD distributions calculated between the primary and all subsequent contours, were used to characterize deformation over the entire treatment course. Comparison of distributions of contours via mean MSDs as opposed to direct comparisons between individual contours was preferred to assess the criteria of creating reasonable poses of patient anatomy verses the same poses as observed in reference images. All synthetic contours exhibited similar magnitudes of interfraction variation to that observed in warped weekly contours (mean MSD differences $<2 \mathrm{~mm}$ for $95 \%$ ) suggesting that the synthetic datasets are indeed reasonable.

While accuracy of weekly registrations was not a primary objective of this study, comparison between warped and manual contours were performed to verify that weekly warped datasets were themselves adequate representations of patient anatomy. This was necessary because weekly warped data was used to validate synthetic datasets; furthermore, the comparison served as a quality assurance measure of registrations that were input to the PCA model. Though a direct comparison between synthetic and manual contours could have been made, stepwise comparisons (i.e. comparing manual to warped and then warped to synthetic) distinguishes between variation due to the statistical model and that due to discrepancies between clinical judgment and image registration. Mean MSD differences between warped and manual contours were less than $2 \mathrm{~mm}$ for $80 \%$ of comparisons, and the largest discrepancies were observed in contours that resided in low contrast regions where inter-observer variation in physician-drawn contours and registration uncertainties are likely.

Additionally, cumulative dose to risk structures on both warped and synthetic datasets were compared under the assumption that similar dose distributions should result over the course of treatment. This comparison provided a more clinically relevant metric for determining the suitability of synthetic 
images. Dosimetric differences were likewise reasonable with $89 \%$ of comparisons differing by less than $5 \%$. The greatest discrepancies were observed in the cord metric (D2) due to the shallow slope in the region of variation; however, dose volume histograms associated with the cord were visually very similar.

Average total and daily volume regression of the gross tumor in synthetic datasets was similar to that reported by others in the literature. Simulated tumor regression for the majority of patients was linear after the first fraction (primary image) with a sometimes dramatic initial deformation (Fig 6). This larger change reflects a discrepancy between modeled and actual data that results from using a linear fit in the sampling of PCA coefficients and the primary image to represent the first fraction of treatment. The implicit assumption is that temporal trends are stable over treatment which may not be true of all patients and thus the primary image may not coincide with the synthetic model. A further limitation associated with imposed linearity, and the fact that synthetic images are deformed instances of the primary image, is that acute patho-anatomical change (e.g. development or resolution of atelectasis) is not easily incorporated into the model. Large systematic discrepancies between the planning and ontreatment images may be mitigated by excluding the former and sampling an additional time-point; however, existing mappings are between the primary image and all synthetic datasets, thus selfconsistent mappings are lost. If observed in actual patient datasets, acute patho-anatomical change may potentially be incorporated into the synthetic model by splitting the data into pre and post groups for individual modeling and then joining the resulting sequences of data; however, this strategy was not implemented here.

A final limitation associated with registration uncertainties in the cranial and caudal extents of the image resulted in artifacts in the same regions of the synthetic imagesets. For all synthetic models in this study, image artifacts fell outside dose-grid regions constructed to contain the whole of the lungs and other relevant risk structures, thus the impact on planning is likely to be negligible.

Statistical modeling of interfraction variation using PCA was first suggested by Sohn et. al. and has been utilized by various authors to predict cumulative dose, evaluate margin adequacy, or construct 
margins based on coverage probabilities, in the context of geometrical uncertainty; however, statistical sampling of PCA coefficients for generating synthetic anatomies implicitly negates temporal trends in the data though such trends may be present and relevant. A PCA model that incorporates temporal trends has been demonstrated by Badawi et. al, though the purpose was to evaluate the ability of the low dimensional model to reconstruct instances of anatomy as opposed to sampling probable poses unobserved in the initial data. In this work we present a method for sampling statistically likely anatomies that preserve temporal trends by performing a linear fit of ordered coefficients and constructing a patient-specific PDF from fit residuals. Previous studies that utilize PCA typically analyze variation in a subset of points associated with contour surfaces; in this study we perform PCA of the entire deformation field to sample global DVFs for deforming images and contours with the intent of creating complete synthetic datasets for virtual trials of adaptive radiotherapy in lung cancer.

\section{Conclusion}

Synthetic datasets that demonstrate relevant temporal trends may be generated by sampling PCA coefficients using a linear fit of time-ordered coefficients and a patient-specific distribution of fit residuals. Sampled datasets consist of self-consistent images, contours, and mappings that are suitable for virtual trials of adaptive radiation therapy.

\section{References}

1. Söhn M, Sobotta B, Alber M. Dosimetric treatment course simulation based on a statistical model of deformable organ motion. Phys Med Biol 2012;57(12):3693-709.

2. Badawi AM, Weiss E, Sleeman WC, Yan C, Hugo GD. Optimizing principal component models for representing interfraction variation in lung cancer radiotherapy. Med Phys 2010;37(9):5080.

3. Ramsey CR, Langen KM, Kupelian PA, Scaperoth DD, Meeks SL, Mahan SL, et al. A technique for adaptive image-guided helical tomotherapy for lung cancer. Int J Radiat Oncol Biol Phys 2006;64(4):1237-44.

4. Woodford C, Yartsev S, Dar AR, Bauman G, Van Dyk J. Adaptive Radiotherapy Planning on Decreasing Gross Tumor Volumes as Seen on Megavoltage Computed Tomography Images. International Journal of Radiation Oncology*Biology*Physics 2007;69(4):1316-22.

5. Guckenberger M, Wilbert J, Richter A, Baier K, Flentje M. Potential of adaptive radiotherapy to escalate the radiation dose in combined radiochemotherapy for locally advanced non-small cell 
lung cancer. Int J Radiat Oncol Biol Phys 2011;79(3):901-8.

6. Weiss E, Fatyga M, Wu Y, Dogan N, Balik S. Dose Escalation for Locally Advanced Lung Cancer Using Adaptive Radiation Therapy With Simultaneous Integrated Volume-Adapted Boost. Int J Radiat Oncol Biol Phys 2013;

7. Glide-Hurst CK, Gopan E, Hugo GD. Anatomic and pathologic variability during radiotherapy for a hybrid active breath-hold gating technique. Int J Radiat Oncol Biol Phys 2010;77(3):910-7.

8. Yan C, Zhong H, Murphy M, Weiss E, Siebers JV. A pseudoinverse deformation vector field generator and its applications. Med Phys 2010;37:1117. 


\section{Appendix III}

Benefits of adaptive radiation therapy in lung cancer as a function of re-planning

frequency 


\section{Benefits of adaptive radiation therapy in lung cancer as a function \\ of re-planning frequency}

Christian Dial", B.Sc., Elisabeth Weiss", M.D., Jeffrey V. Siebers`, Ph.D., Geoffrey D. Hugo", Ph.D.

"Department of Radiation Oncology, Virginia Commonwealth University, Richmond, VA, 23298

'Department of Radiation Oncology, University of Virginia, Charlottesville, VA, 22908

\section{Corresponding author}

Geoffrey D. Hugo, Ph.D.

Department of Radiation Oncology

Virginia Commonwealth University

401 College Street

PO Box 980058

Richmond, VA 23298

Phone: 804-628-3457

Fax: 804-628-0271

E-mail: gdhugo@vcu.edu

\section{Conflicts of interest}

The authors have no actual or potential conflicts of interest to disclose. 


\begin{abstract}
Purpose: To quantify the potential benefit associated with daily replanning in lung cancer in terms of normal tissue dose sparing, and to characterize the tradeoff between adaptive benefit and replanning frequency.
\end{abstract}

Methods: A set of synthetic images and contours derived from weekly images of 12 patients undergoing radiation therapy treatment for non-small cell lung cancer (NSCLC) are generated for each fraction of treatment using principal component analysis (PCA) in a way that preserves temporal anatomical trends (e.g. tumor regression). Daily synthetic images and contours are used to simulate 4 different treatment scenarios: 1) a "no-adapt" scenario that simulates delivery of an initial plan throughout treatment, 2) a "mid-adapt" scenario that implements a single replan for fraction 18, 3) a "weekly-adapt" scenario that simulates weekly adaptations, and 4) a "full-adapt" scenario that simulates daily replanning. An initial intensity modulated radiation therapy (IMRT) plan is created for each patient, and replanning is carried out in an automated fashion by re-optimizing beam apertures and weights. Dose is calculated on each image and accumulated to the first in the series using deformable mappings utilized in synthetic image creation for comparison between simulated treatments.

Results: Target coverage was maintained and cord tolerance was not exceeded for any of the adaptive simulations. Average reductions in mean lung dose and volume of lung receiving 20 Gy or more $\left(\mathrm{V} 20_{\text {lung }}\right)$ were $65 \pm 49 \mathrm{cGy}(\mathrm{p}=0.00001)$ and $1.1 \pm 1.2 \%(\mathrm{p}=0.0006)$ respectively for all patients. The largest reduction in mean lung dose for a single patient was $162 \mathrm{cGy}$ which allowed an isotoxic escalation of target dose of $1668 \mathrm{cGy}$. Average reductions in cord max dose, mean esophageal dose, dose received by 66 percent of the heart (D66 heart $_{\text {t }}$, and dose received by 33 percent of the heart (D33 $3_{\text {heart }}$ ), were $158 \pm 280 \mathrm{cGy}, 117 \pm 121 \mathrm{cGy}, 37 \pm 77 \mathrm{cGy}$, and $99 \pm$ 120 cGy respectively. Average incremental reductions in mean lung dose for the mid-adapt, weekly-adapt, and full-adapt treatments were 38 cGy, $18 \mathrm{cGy}$, and 8 cGy respectively. Incremental reductions in mean esophageal dose for the same treatments were $57 \mathrm{cGy}, 37 \mathrm{cGy}$, 
and 23 cGy. Reductions in mean lung dose and mean esophageal dose for the full-adapt

treatment were correlated with the absolute decrease in the planning target volume $(r=0.34$ and $r$ $=0.26$ ).

Conclusion: Adaptive radiation therapy for lung cancer yields clinically relevant reductions in normal tissue doses for frequencies of adaptation ranging from a single replan up to daily replanning. Increased frequencies of adaptation result in additional benefit while magnitude of benefit decreases.

\section{Key words}

Non-small-cell lung cancer, adaptive radiation therapy, tumor regression

\section{Introduction}

Lung cancer is one of the leading causes of death for both men and women with approximately 570,000 deaths reported for the year 2009 in the United States. ${ }^{[1]}$ For the year 2013, The American Cancer Society estimated approximately 580,000 cancer related deaths and projected nearly 230,000 of those to be associated with lung cancer making it the number one cause of cancer-related death. ${ }^{[2]}$ Radiotherapy is a mainstay of treatment and is the preferred modality for approximately $40 \%$ of newly diagnosed patients $;^{[3]}$ however, prognosis is poor for those receiving radiation therapy with 5 year survival around $17 \%$ as reported by The National Cancer Institute. ${ }^{[4]}$

Local control and survival have been shown to improve with increased doses,${ }^{[5,6]}$ but for many patients deliverable dose is limited by associated normal tissue toxicity. Thus, strategies that limit normal tissue exposure while allowing isotoxic escalation of the prescription dose are desirable. 
Re-planning to accommodate inter-fraction variation in lung cancer (i.e. regression of the gross tumor volume) to reduce treatment margins and allow for dose escalation has been investigated by various authors. ${ }^{[7-10]}$ These works implement various replanning schedules ranging from single to weekly adaptations and demonstrate a patient-specific benefit for adaptive plans in the context of tumor regression. While a variety of schedules have been implemented by the respective authors, systematic comparisons between schedules of varying adaptive frequency have not been performed; furthermore, the nature of tradeoff between replanning frequency and adaptive benefit is not understood. A final limitation of current studies is that they implement at most weekly adaptation, thus the benefit associated with daily replanning remains unclears.

The purpose of this work is to quantify the benefit associated with various frequencies of adaptation, up to daily replanning, for a cohort of lung cancer patients, and to characterize the tradeoff between adaptive benefit and replanning frequency.

\section{Methods}

Four treatment schedules that implement different frequencies of replanning are simulated for a group of lung cancer patients. Simulations are based on a set of synthetic images and contours that are deformed instances of the first in a sequence of weekly images, and are generated using principal component analysis (PCA). Daily synthetic images are used for simulated re-planning and daily dose calculation after which dose is accumulated back to the first image for comparison of simulated schedules.

\section{Synthetic datasets}

Generation of synthetic datasets for each fraction of treatment from an existing sequence of temporally ordered images has been described previously. (REF to PCA paper) Briefly, deformable registrations between the first and all subsequent images in a temporally ordered set result in a set of mappings that effectively track variation. Displacement vector fields (DVF) that 
contain translations in the $\mathrm{x}, \mathrm{y}$, and $\mathrm{z}$ directions are reshaped into a single column vector and concatenated into a joint variation matrix with homologous points residing along rows of the matrix. After mean-correcting the matrix by subtracting the row means from each row, the matrix is decomposed into a set of basis vectors and weighting coefficients using PCA. Synthetic weighting coefficients for each basis vector are generated for arbitrary time-points by evaluating a linear fit of coefficients associated with existing images and adding a random portion sampled from a patient-specific distribution of fit residuals approximated using kernel density estimation. Sampling in this fashion preserves temporal trends in the data (e.g. tumor regression) and resulting coefficients are utilized to create synthetic DVFs associated with desired time-points. A pseudo-inverse DVF is generated as described by Yan et al. ${ }^{[1]}$ and is used to deform the first image in the series along with associated contours.

Synthetic datasets generated in this work are based on an existing set of patient data corresponding to 12 non-small-cell lung cancer (NSCLC) patients undergoing external beam radiation therapy for stage II and III disease; patient characteristics are summarized in Table 1. For each patient $4-6$ weekly helical CTs were obtained under an active breathing control (ABC) protocol to minimize respiratory motion, and structures of interest were delineated by a qualified physician including: gross tumor volume (GTV), lungs, spinal cord, heart, and esophagus. Registrations between weekly images utilized in the PCA analysis were performed using the Demons algorithm as implemented in the Pinnacle treatment planning system (Philips Oncology, Fitchburg, WI). 


\begin{tabular}{|c|c|c|c|c|c|c|c|c|c|}
\hline patient & stage & location & nodes & $\begin{array}{l}\text { tumor vol } \\
\text { (cc) }\end{array}$ & patient & stage & location & nodes & $\begin{array}{l}\text { tumor vol } \\
\text { (cc) }\end{array}$ \\
\hline 1 & IIIA & LUL & $\mathrm{Y}$ & 24 & 9 & IIIA & RUL & $\mathrm{Y}$ & 40 \\
\hline 3 & IIIB & Bilateral & $\mathrm{Y}$ & 100 & 14 & IIIA & RML & $\mathrm{Y}$ & 34 \\
\hline 4 & IIB & LLL & $\mathrm{N}$ & 65 & 17 & IIIA & RUL & $\mathrm{Y}$ & 216 \\
\hline 5 & IIIB & RUL & $\mathrm{Y}$ & 1 & 18 & IIIB & RUL & $\mathrm{N}$ & 58 \\
\hline 6 & IIIA & RLL & $\mathrm{N}$ & 242 & 20 & NA & LLL & Y & 47 \\
\hline 8 & IIB & RUL & $\mathrm{N}$ & 11 & 21 & NA & LUL & $\mathrm{N}$ & 86 \\
\hline
\end{tabular}

Table 1. Patient characteristics of 12 non-small-cell lung cancer patients used to generate a set of synthetic datasets. LUL = left upper lobe; $\mathrm{LLL}=$ left lower lobe; $\mathrm{RUL}=$ right upper lobe; $\mathrm{RLL}=$ right lower lobe; $\mathrm{RML}=$ right middle lobe.

PCA analysis and synthetic data generation resulted in a set of self-consistent images, contours, and mappings, corresponding to each fraction in a typical 35 fraction schedule. For patients with delineated nodal volumes, two sets of simulations were carried out, the first of which excluded nodal targets and the second of which included them; simulations that included nodal volumes are referenced by appending an " $\mathrm{N}$ " to the patient identifier. For the single patient that presented with bilateral tumors, treatments are derived based on each tumor separately. This process resulted in 20 different sets of target contours based on 12 sequences of synthetic images for use in treatment simulations.

\section{Planning}

Intensity modulated radiation therapy (IMRT) plans are developed for each patient and target based on a synthetic image corresponding to the first fraction. Planning assumed breathhold and image guidance throughout treatment to limit geometric uncertainty associated with respiratory motion and patient setup. A margin of $5 \mathrm{~mm}$ was implemented to account for subclinical disease, and PTV margins of $7 \mathrm{~mm}$ in the superior-inferior direction, and $5 \mathrm{~mm}$ in the axial plane, were utilized as recommended in radiation therapy oncology group (RTOG) protocol 0839 for plans that implement breath-hold and image guidance.

After construction of target volumes, 5-8 coplanar beams were arranged around the patient in a manner as to avoid directly traversing risk structures when possible. Nominal beam energy was set to 6MV and beams were prescribed to a dose of $66 \mathrm{~Gy}$. 
A simplified set of IMRT objectives are utilized in plan optimization that focus on target coverage and dose conformality as opposed to implementing individual objectives for each risk structure. A $2 \mathrm{~cm}$ thick "rind" region of interest (ROI) beginning $2 \mathrm{~mm}$ from the surface of the PTV was utilized to suppress dose to surrounding risk structures and guide the optimization to a conformal solution. Plans were deemed acceptable if they met criteria associated with PTV coverage and if cord tolerance was not exceeded. Specifically, minimum dose to any volume associated with the PTV of at least $0.03 \mathrm{cc}$ in magnitude (PTV min dose) was not to fall below $95 \%$ of the prescription dose, $95 \%$ of the PTV dose (D95 $5_{\text {PTV }}$ ) was to be greater than or equal to the prescription, and max dose to any volume of $0.03 \mathrm{cc}$ associated with the spinal cord (cord max dose) was not to exceed 50.5 Gy. In cases where spinal cord tolerances were exceeded, an additional constraint was added to the set of objectives and an additional optimization was carried out. Additional criteria were considered in plan evaluation but were allowed to vary within clinical judgment; a summary of normal tissue planning criteria utilized in this work is given in Table 2.

\begin{tabular}{ccc}
\hline & NORMAL TISSUE PLAN CRITERIA \\
\hline \hline ROI & metric & value \\
\hline cord & max dose & $5050 \mathrm{cGy}$ \\
lungs & mean dose & $2000 \mathrm{cGy}$ \\
lungs & V20 & $37 \%$ \\
esophagus & mean dose & $4000 \mathrm{cGy}$ \\
heart & max dose to $1 / 3$ of volume & $6000 \mathrm{cGy}$ \\
heart & max dose to $2 / 3$ of volume & $4500 \mathrm{cGy}$ \\
\hline \hline
\end{tabular}

Table 2. Plan criteria associated with risk structures. * Plans not meeting this criteria were not deemed acceptable.

\section{Treatment simulation}

Treatment simulations for each of the four adaptation schedules and patients are carried out in an automated fashion using a research version of the Pinnacle treatment planning system (TPS). Simulated treatments include: 1) a "no-adapt" treatment for which the initial plan is delivered throughout the treatment course; 2) a "mid-adapt" treatment that implements 
replanning a single time for fraction 18 ; 3) a "weekly-adapt" treatment that implements

replanning for fractions $6,11,16,21,26$, and 31; and 4) a "full-adapt" treatment that implements daily replanning for each fraction.

Initial beam angles are held constant throughout treatment, and re-planning is carried out for adaptive fractions by re-optimizing beam apertures and monitor units (MU) using the same set of objectives and weights implemented in the initial plan; the latter is important to ensure that benefit is due to anatomical variability and not to a re-appropriation of objective weights. For non-adaptive fractions, the most recent plan (i.e. configuration of beam apertures and MUs) is transferred to the corresponding fraction image, and dose is recalculated on the same. In all cases, the beam isocenter is set to the centroid of the propagated clinical treatment volume (CTV) simulating a soft-tissue image-guided setup.

After simulation, dose is accumulated from each fraction to the first image in the series using the pseudo-inverse of the same DVF utilized in synthetic image creation.

\section{Adaptive benefit}

Benefit in this study is primarily quantified in terms of reductions in planning metrics associated with dose to lungs though changes are also reported for cord, esophagus, and heart. Comparisons are made between full-adapt and no-adapt treatments along with incremental comparisons of each of the three adaptive treatments to the treatment implementing the next lowest amount of re-plans, e.g. the full-adapt treatment is compared to the weekly-adapt, and the weekly-adapt simulation is compared to the mid-adapt treatment. To ensure that perceived benefits do not come at the expense of plan acceptability criteria, coverage of the CTV, and spinal cord tolerances, are first evaluated for all simulated treatments.

Isotoxic dose escalation afforded by achieved reductions are estimated by scaling dose distributions for the full-adapt treatment until MLD matches that of the no-adapt plan. Differences between the full-adapt cumulative target dose and scaled full-adapt cumulative target dose are reported. 
Paired t-tests are calculated for all inter-treatment dose-metric comparisons. As a conservative measure to control for the familywise error rate associated with multiple comparisons, a Bonferroni correction is utilized. Briefly, when carrying out multiple comparisons, the probability of committing a type I error increases with the amount of comparisons. The Bonferroni correction entails using a statistical significance of $\alpha / n$ for each test where $n$ is the number of comparisons.

\section{Results}

\section{Plan acceptance}

Plan acceptance criteria associated with target coverage and spinal cord tolerance for the initial plan was maintained for all patients and simulations. $\mathrm{D} 95_{\mathrm{CTV}}$ was greater than or equal to the prescription dose of $66 \mathrm{~Gy}$ with percent changes from the no-adapt simulation equal to $0.4 \pm$ $1.4 \%, 0.4 \pm 1.0 \%$, and $0.4 \pm 1.0 \%$ for the full-adapt, weekly-adapt, and mid-adapt treatments respectively. Mean CTV min dose was $65 \pm 3$ Gy for the no-adapt simulations and was $66 \pm 2$ Gy for each of the adaptive treatments. Spinal cord tolerance of 50.5 Gy was initially exceeded for four patients using the simplified objective scheme but was brought within range after adding an additional optimizer constraint for these patients to account for cord dose directly.

Differences between no-adapt and full-adapt normal-tissue dose metrics are listed for all patients in Table 3. A maximum decrease in mean lung dose of -162 cGy was observed with an average decrease of -65 cGy over the patient population. V20 showed little variation between no-adapt and full-adapt simulations ranging between $-2.96 \%$ and $1.06 \%$ with a mean decrease of $-1.1 \%$. Average change in mean esophageal dose was -117 cGy with a maximum decrease of -415 cGy, and average decreases of -37 cGy and -99 cGy were observed for heart D66 and D33 respectively. Differences between reported metrics for full-adapt and no-adapt simulations were 
statistically significant using the Bonferroni criterion $(\mathrm{p}<0.008)$ for mean lung dose, lung V20, mean esophageal dose, and heart D33.

\begin{tabular}{|c|c|c|c|c|c|c|}
\hline & $\begin{array}{l}\text { cord max } \\
\text { (cGy) }\end{array}$ & $\begin{array}{l}\text { mean lung } \\
\text { dose (cGy) }\end{array}$ & $\begin{array}{c}\text { lung V20 } \\
(\% \text { vol })\end{array}$ & $\begin{array}{c}\text { mean esophagus } \\
\text { dose (cGy) }\end{array}$ & $\begin{array}{c}\text { heart D66 } \\
\text { (cGy) }\end{array}$ & $\begin{array}{l}\text { heart D33 } \\
\text { (cGy) }\end{array}$ \\
\hline PT1 & 40 & -48 & -1.39 & -415 & -2 & -3 \\
\hline PT1N & 132 & -20 & -0.61 & -111 & 1 & -1 \\
\hline PT3L & 259 & -69 & -1.12 & -106 & -3 & -54 \\
\hline PT3LN & -560 & -6 & -0.15 & -68 & -5 & -151 \\
\hline PT3R & -188 & -106 & -2.01 & -128 & -9 & -128 \\
\hline PT3RN & -77 & -117 & -2.29 & -55 & -16 & -320 \\
\hline PT4 & -243 & -37 & 0.69 & -72 & -43 & -148 \\
\hline PT5 & 119 & 14 & 0.27 & -2 & 0 & 0 \\
\hline PT6 & -325 & -43 & -0.44 & -144 & -337 & -280 \\
\hline PT8 & -656 & -162 & -2.76 & -186 & -17 & -49 \\
\hline РT9 & -250 & -115 & -2.96 & -2 & -2 & -22 \\
\hline PT9N & -231 & -80 & -0.7 & 66 & 1 & 12 \\
\hline PT14 & -187 & -92 & -1.96 & -159 & -104 & -170 \\
\hline PT14N & -91 & -37 & -0.7 & -15 & 0 & 38 \\
\hline PT17 & -8 & -54 & 1.06 & -294 & -27 & -80 \\
\hline PT17N & -656 & -100 & -1.72 & -253 & -14 & -54 \\
\hline PT18 & 65 & 8 & 0 & 26 & 15 & 69 \\
\hline PT20 & 311 & 10 & 0.05 & 1 & -7 & -7 \\
\hline PT20N & -363 & -64 & -2.43 & -231 & -39 & -104 \\
\hline PT21 & 104 & -106 & -2.22 & -47 & -87 & -367 \\
\hline mean change & -158 & -65 & -1.1 & -117 & -37 & -99 \\
\hline $\mathrm{p}$ & 0.04 & 0.00001 & 0.0006 & 0.0005 & 0.05 & 0.002 \\
\hline
\end{tabular}

Table 3. Absolute changes in full-adapt normal-tissue dose metrics compared to the no-adapt simulation. Average changes are listed below along with p-values resulting from paired t-tests comparing full-adapt and no-adapt metrics.

Benefit associated with re-planning at different frequencies is illustrated for each normal tissue metric in Fig. 1. Comparisons of adaptive simulations to next treatments of lower replanning frequency (i.e. full-adapt to weekly-adapt, weekly-adapt to mid-adapt, and mid-adapt to no-adapt) are listed in Table 4. Statistically significant differences between all adaptive schedules and the no-adapt simulation were observed for mean lung dose, and lung V20. 

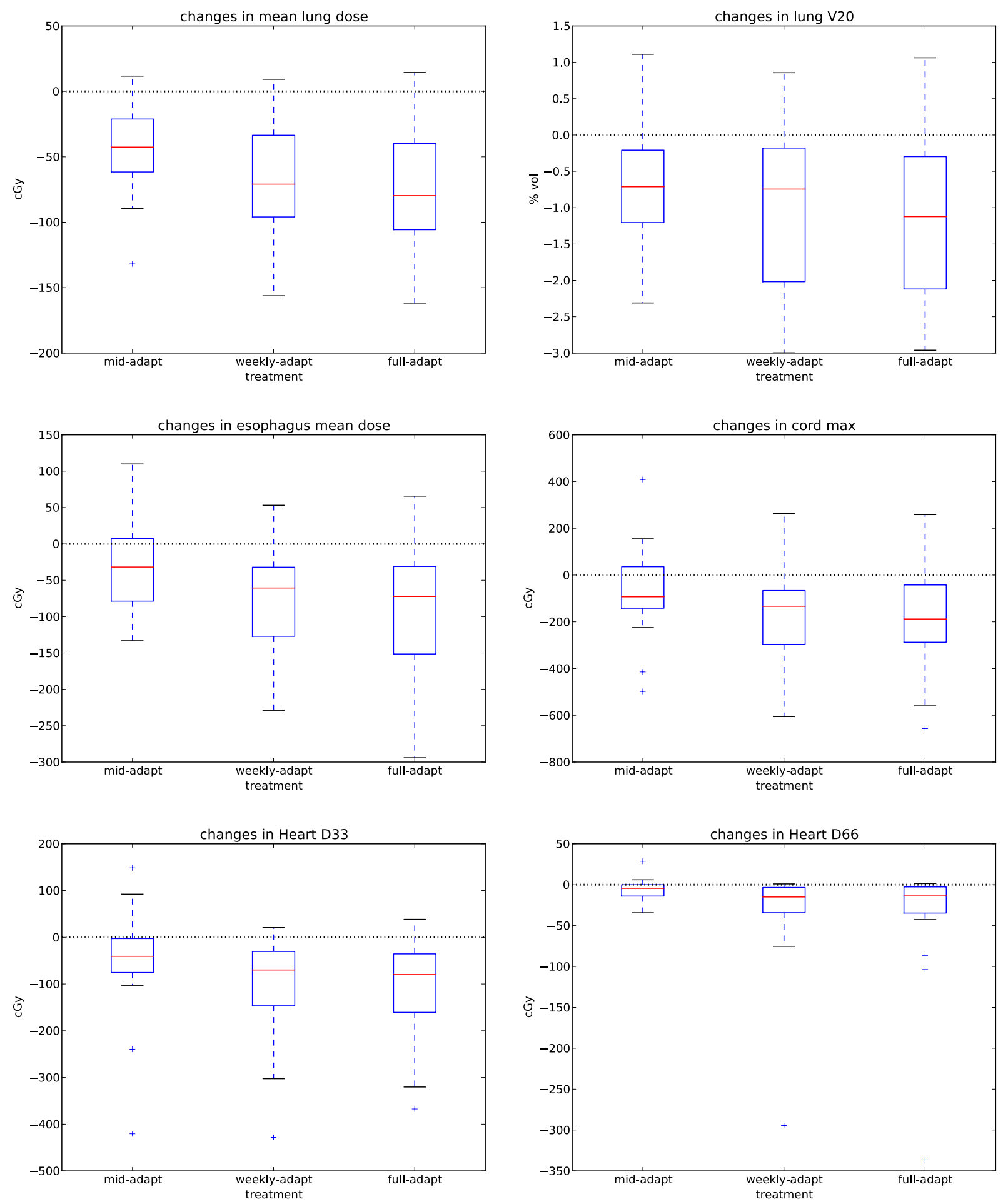

Fig. 1. Distributions of changes in normal-tissue dose metrics compared to the no-adapt simulation for mid-adapt, weekly-adapt, and full-adapt treatments. Increases in planning frequency resulted in further reductions of dose for the majority of structures. *Differences between adaptive simulations and no-adapt treatment statistically significant using Bonferroni criteria $(p<0.008)$. 
AVERAGE CHANGE IN NORMAL TISSUE DOSE

\begin{tabular}{ccccccc}
\hline \hline & mid - no & $\mathrm{p}$ & weekly - mid & $\mathrm{p}$ & full - weekly & $\mathrm{p}$ \\
\hline cord max & $-52 \mathrm{cGy}$ & 0.3 & $-104 \mathrm{cGy}$ & 0.01 & $-1 \mathrm{cGy}$ & 0.9 \\
mean lung dose & $-38 \mathrm{cGy}$ & 0.0001 & $-18 \mathrm{cGy}$ & 0.002 & $-8 \mathrm{cGy}$ & 0.03 \\
lung V20 & $-0.68 \%$ & 0.004 & $-0.37 \%$ & 0.006 & $-0.09 \%$ & 0.4 \\
mean esophagus dose & $-57 \mathrm{cGy}$ & 0.03 & $-37 \mathrm{cGy}$ & 0.02 & $-23 \mathrm{cGy}$ & 0.01 \\
heart D66 & $-7 \mathrm{cGy}$ & 0.09 & $-25 \mathrm{cGy}$ & 0.1 & $-5 \mathrm{cGy}$ & 0.2 \\
heart D33 & $-49 \mathrm{cGy}$ & 0.09 & $-45 \mathrm{cGy}$ & 0.03 & $-5 \mathrm{cGy}$ & 0.4 \\
\hline \hline
\end{tabular}

Table 4. Incremental differences between dose metric averages for adaptive schedules and treatments of next lowest replanning frequency (e.g. full-adapt and weekly-adapt). Associated p-values resulting from paired t-tests listed to the right of each comparison.

The relationship between absolute decreases in PTV volume and decreases in both mean lung dose and mean esophageal dose are illustrated in Fig. 2; Pearson correlation coefficients were 0.34 and 0.26 for each comparison respectively.

(a)

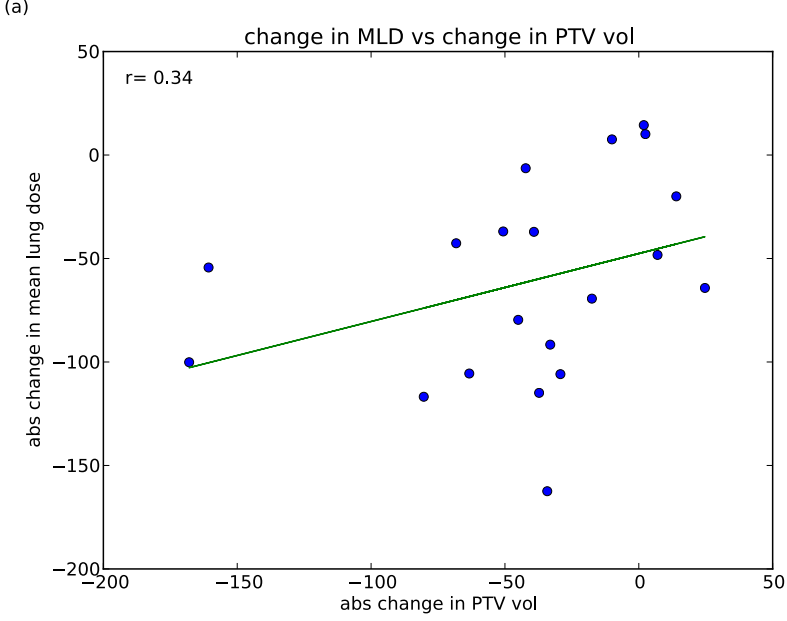

(b)

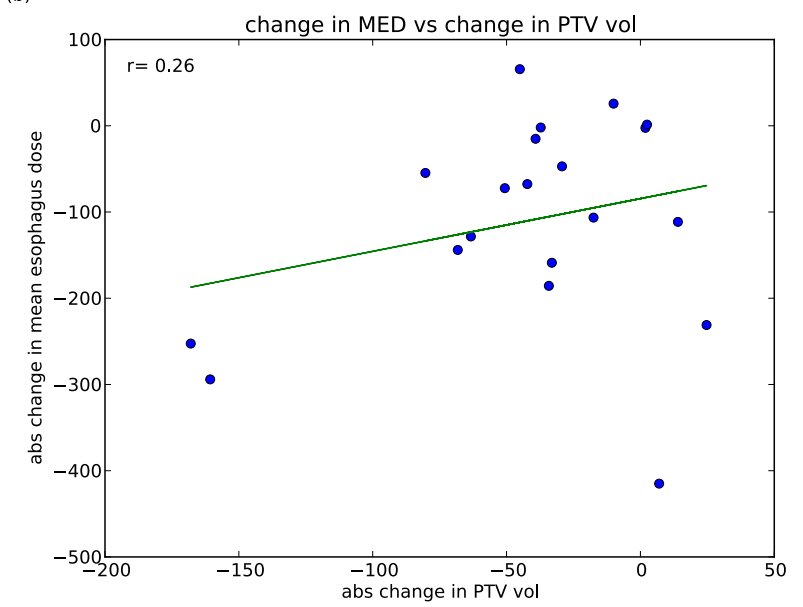

Fig. 2 Correlation of absolute changes in PTV volume with changes in both mean lung dose (a) and mean esophageal dose (b) as observed in the full-adapt treatment simulation. Decreases in PTV volume were moderately correlated with reductions in both metrics; Pearson correlation coefficients (r) listed in upper left of each plot.

Isotoxic escalation of dose resulted in average increases in CTV D95 of 294 (0 - 1304)

cGy, $381(10$ - 1593) cGy, and $441(31$ - 1668) cGy for the mid-adapt, weekly-adapt, and fulladapt simulations respectively. 


\section{Discussion}

In this work we utilized a set of daily synthetic lung images and contours that exhibited temporal anatomical trends (e.g. tumor regression) to simulate different adaptive schedules with the objective of quantifying the benefit of a full-adapt treatment in lung cancer and to characterize the relationship between adaptive benefit and replanning frequency.

Daily adaptation to regressing tumor volumes yielded statistically significant reductions in dose for all reported averages of normal-tissue metrics considered in this work; however, reductions were not realized for all patients, reinforcing the notion that benefit associated with adaptation is ultimately patient dependent. For the three patients (PT5, PT18, and PT20) exhibiting no reduction in mean lung dose as a result of daily adaptation, two had PTVs that did not decrease in volume over the course of treatment; the third exhibited an overall decrease in PTV with an initial pattern of regression followed by a period of increasing volume around fraction 22 .

Using an isotoxic criteria based on mean lung dose, average allowable dose escalation was 441 cGy for the full-adapt plan, without exceeding cord tolerances, resulting from an average decrease in mean lung dose of 5\%; a maximum escalation of 17 Gy was achieved for a single patient. In a study consisting of 12 patients that implemented replanning twice at weeks 3 and 5, Guckenberger et. al. reported an average escalation of $7 \mathrm{~Gy}$ based on a reduction in mean lung dose of approximately $8 \%{ }^{[9]}$ In another study which adapted at weeks 2 and 4 conducted by Weiss et. al, an average increase of $13.4 \mathrm{~Gy}$ with a maximum of $23.4 \mathrm{~Gy}$ was achieved.$^{[10]}$ These values are larger than those reported here for daily adaptation; differences may be attributed to volumes used to estimate escalation i.e. in this work dose was escalated to the CTV as opposed to primary tumor.

Incremental reductions in all average dose metrics were observed with each increase in replanning frequency (Table 4); however, the magnitude of each reduction decreased with each step. For mean lung dose, $60 \%$ of the average total reduction that resulted from the full-adapt 
plan was realized after a single mid-treatment adaptation, and $88 \%$ was realized using weekly adaptation. For mean esophageal dose, $50 \%$ of benefit was achieved with a single mid-treatment adaptation, and $80 \%$ was realized after weekly adaptation. Average increases in allowable target dose as a function of replanning frequency are summarized in Fig. 3. Approximately $65 \%$ of the potential dose escalation was achievable with a single mid-treatment adaptation, and about $85 \%$ was achievable with weekly adaptation. The last $15 \%$ of the reported average benefit is associated with a 6 fold increase in workload over weekly replanning which itself represents a large increase in cost over a single adaptation. Considerations of this sort suggest that the workload associated with daily adaptation outweighs additional benefit, and weekly adaptation would be most favorable for the majority of patients. However, as the workload associated with planning decreases through the development of automatic methods, daily replanning may be justified.

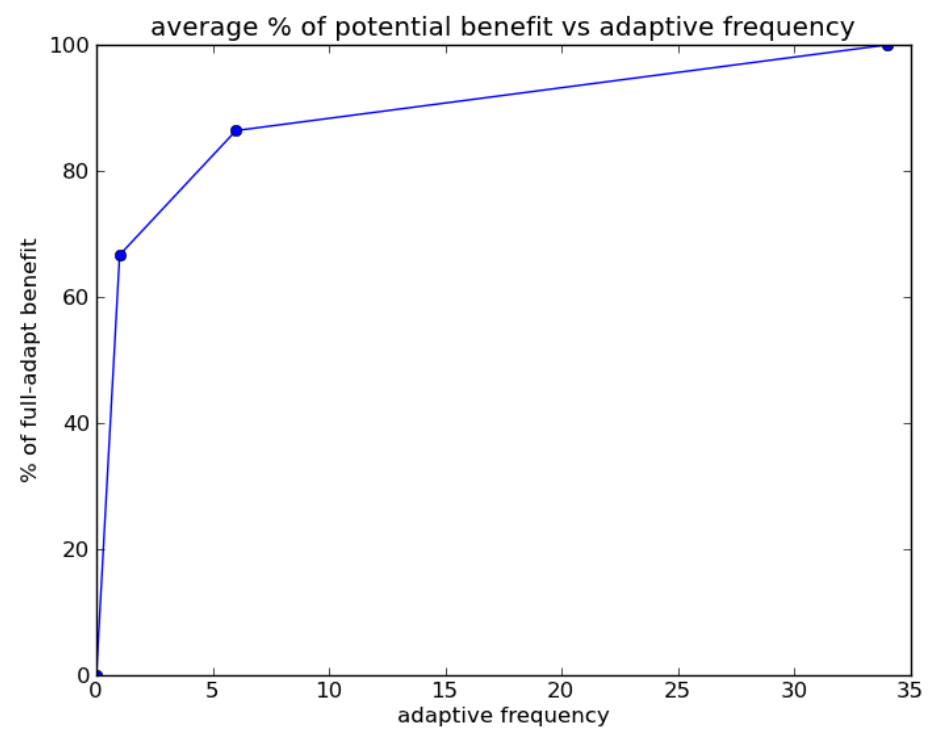

Fig. 3 Percent of potential benefit (i.e. allowable dose to target) as a function of replanning frequency. On average, $65 \%$ of benefit was achieved with a single mid-treatment adaptation, and $85 \%$ was realized after weekly adaptation.

Due to the sampling method employed in the generation of synthetic datasets, trends of variation were devoid of large acute change which represents an important limitation of the study. In this work we simulated replanning at regular intervals; however, such a method is ultimately naïve and acute variation may warrant a more tailored approach. 
Though correlation was modest, reductions in both mean lung dose and mean esophageal dose were related to the absolute change in PTV $\left(\mathrm{r}_{\mathrm{MLD}}=0.34\right.$ and $\left.\mathrm{r}_{\mathrm{MED}}=0.26\right)$. Additional correlations of adaptive benefit were investigated including nodal status and size of the initial PTV, however, no relationship was observed. Ultimately, the small sample size prevented a systematic evaluation of benefit versus patient characteristics; however, the methods employed in this work could be used to carry out such investigations. Enumerating and articulating patient characteristics related to adaptive benefit will be the work of future studies.

\section{Conclusion}

Daily adaptation in lung cancer radiotherapy produced significant reductions in normaltissue dose metrics which allowed clinically relevant increases in target dose using an isotoxic mean lung dose criteria. Normal-tissue dose sparing was likewise observed for a single midtreatment adaptation and weekly replanning when compared to a no-adapt simulation. Incremental reductions were realized for mean lung dose and mean esophageal dose with each increase in replanning frequency while magnitude of each reduction decreased.

\section{References}

1. Heron M. Deaths: Leading Causes for 2009. National Vital Statistics Reports; 2012.

2. Siegel R, Naishadham D, Jemal A. Cancer statistics, 2013. CA Cancer J Clin 2013;63(1):11-30.

3. Halperin EC, Pérez CA, Brady LW. Perez and Brady's Principles and Practice of Radiation Oncology. Lippincott Williams \& Wilkins; 2008.

4. Howlader N, Noone AM, Krapcho M, Garshell J, Neyman N, Altekruse SF, et al. SEER Cancer Statistics Review, 1975-2010.

5. Mehta M, Scrimger R, Mackie R, Paliwal B, Chappell R, Fowler J. A new approach to dose escalation in non-small-cell lung cancer. Int J Radiat Oncol Biol Phys 2001;49(1):23-33.

6. Kong F-M, Haken Ten RK, Schipper MJ, Sullivan MA, Chen M, Lopez C, et al. Highdose radiation improved local tumor control and overall survival in patients with inoperable/unresectable non-small-cell lung cancer: Long-term results of a radiation dose 
escalation study. International Journal of Radiation Oncology*Biology*Physics

2005;63(2):324-33.

7. Ramsey CR, Langen KM, Kupelian PA, Scaperoth DD, Meeks SL, Mahan SL, et al. A technique for adaptive image-guided helical tomotherapy for lung cancer. Int J Radiat Oncol Biol Phys 2006;64(4):1237-44.

8. Woodford C, Yartsev S, Dar AR, Bauman G, Van Dyk J. Adaptive Radiotherapy Planning on Decreasing Gross Tumor Volumes as Seen on Megavoltage Computed Tomography Images. International Journal of Radiation Oncology*Biology*Physics 2007;69(4):131622.

9. Guckenberger M, Wilbert J, Richter A, Baier K, Flentje M. Potential of adaptive radiotherapy to escalate the radiation dose in combined radiochemotherapy for locally advanced non-small cell lung cancer. Int J Radiat Oncol Biol Phys 2011;79(3):901-8.

10. Weiss E, Fatyga M, Wu Y, Dogan N, Balik S. Dose Escalation for Locally Advanced Lung Cancer Using Adaptive Radiation Therapy With Simultaneous Integrated VolumeAdapted Boost. Int J Radiat Oncol Biol Phys 2013;

11. Yan C, Zhong H, Murphy M, Weiss E, Siebers JV. A pseudoinverse deformation vector field generator and its applications. Med Phys 2010;37:1117. 


\section{Appendix IV}

Absolute volume measurements of synthetic gross, clinical, and planning target volumes associated with each fraction of treatment.

GTV: gross tumor volume (blue)

CTV: clinical target volume (green)

PTV: planning target volume (maroon) 

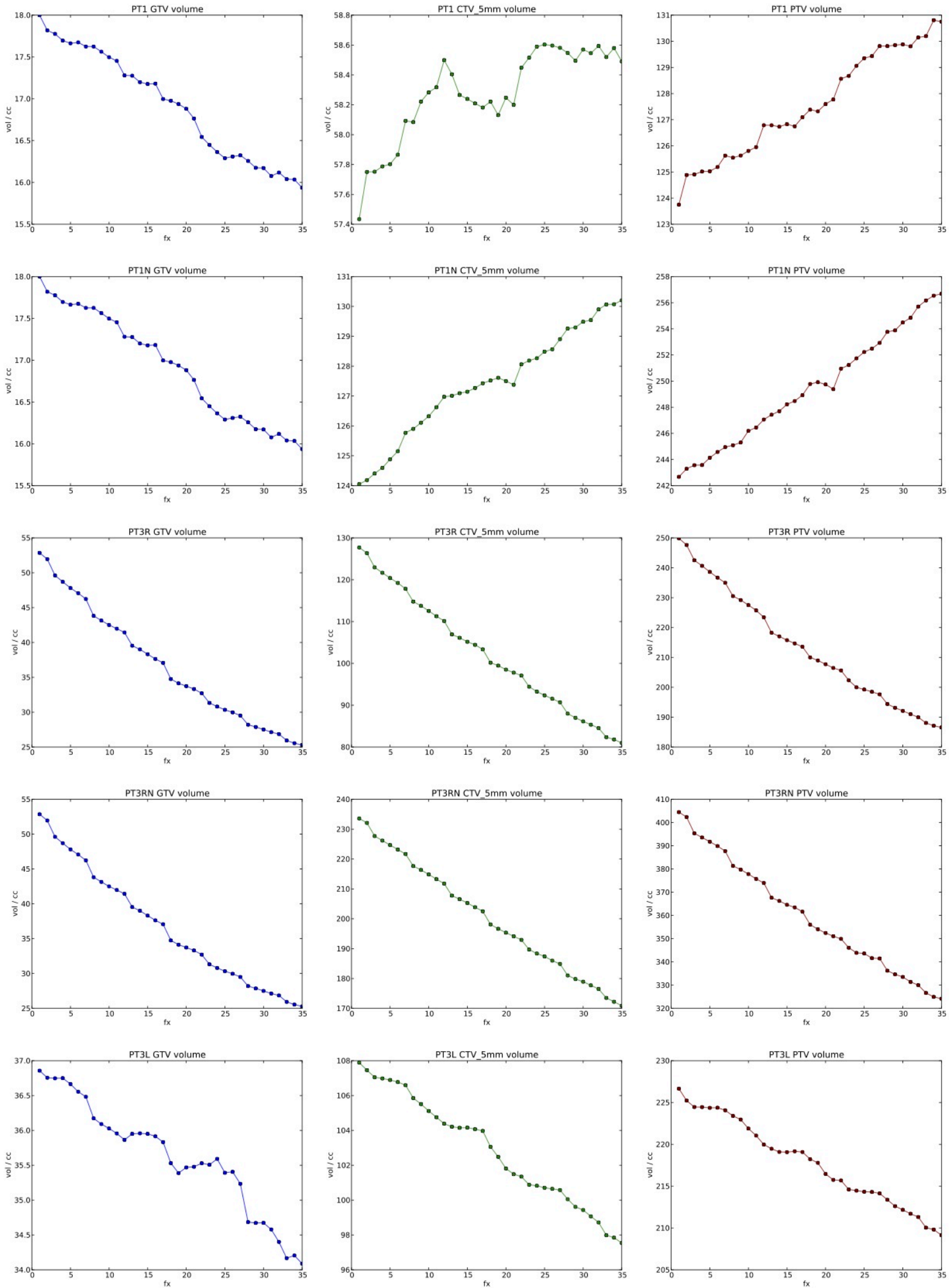

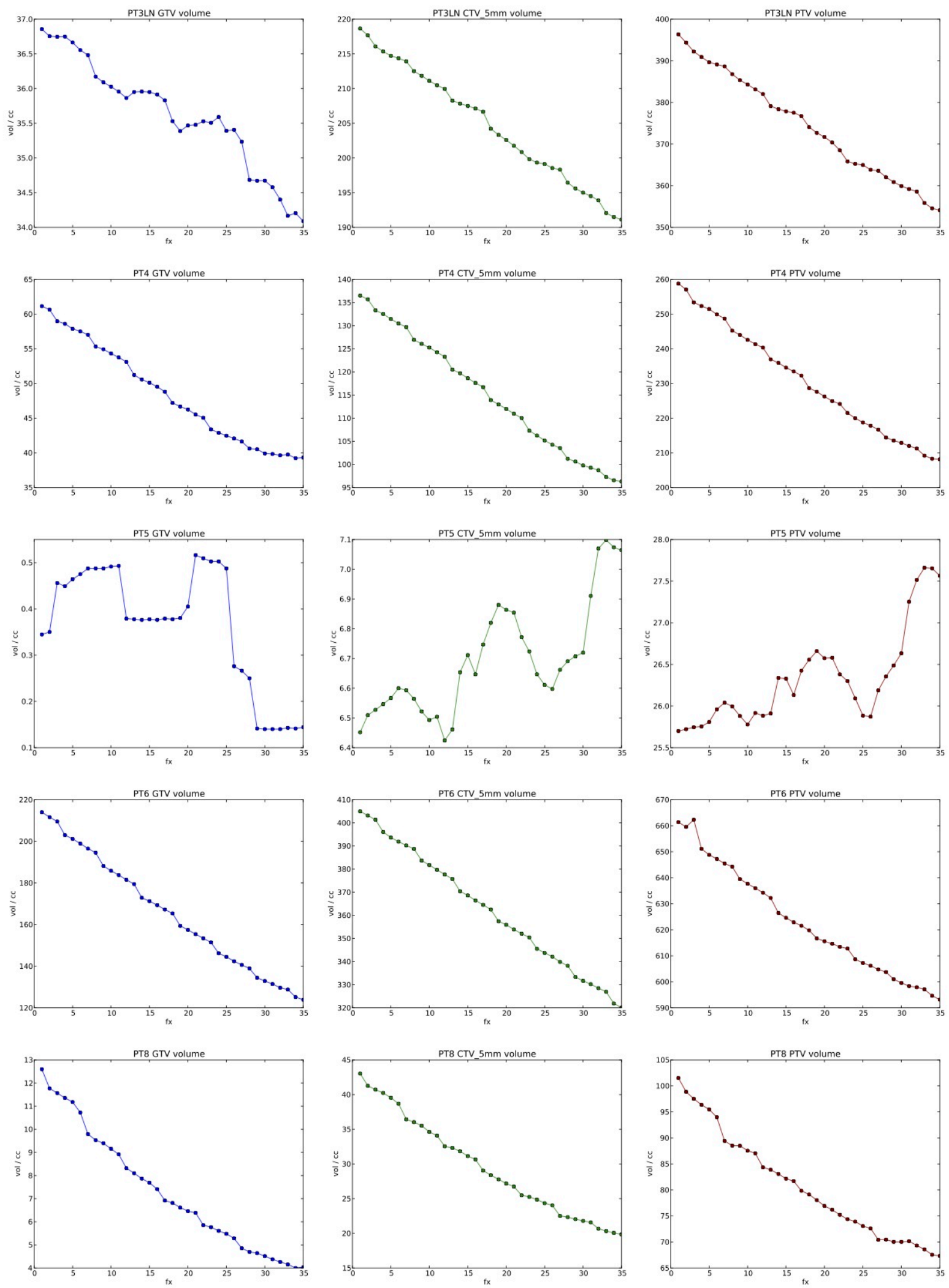

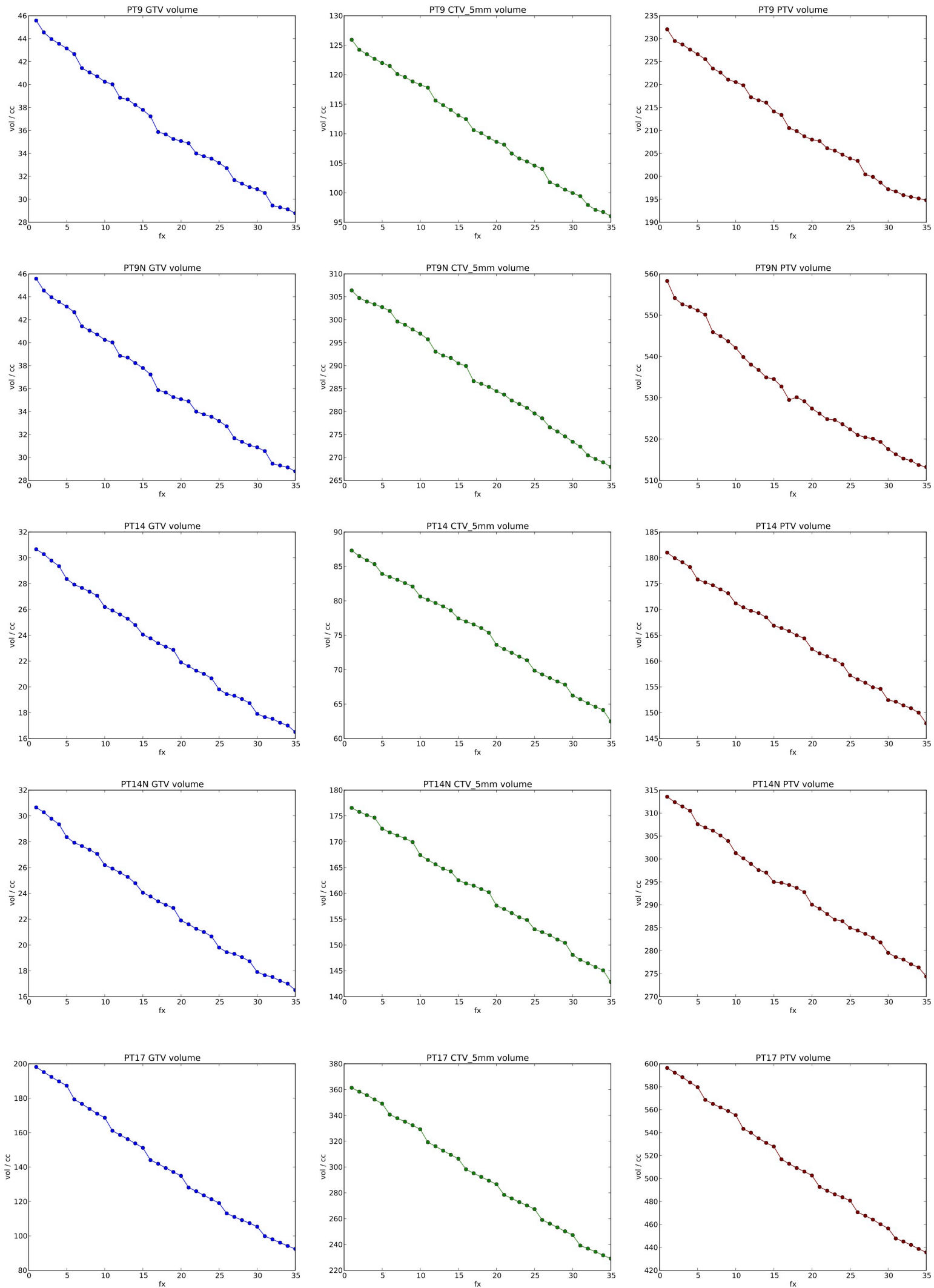

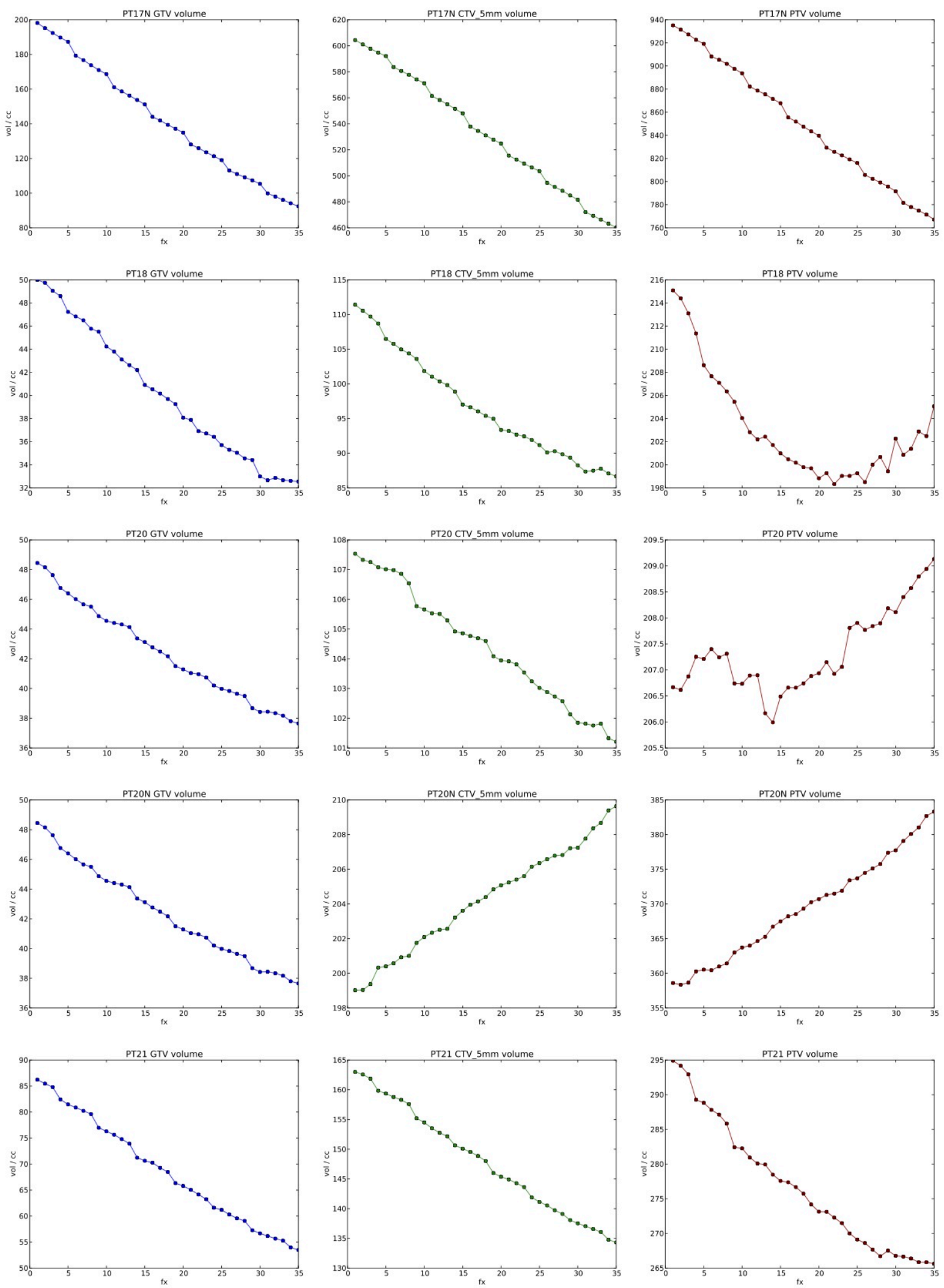


\section{Appendix V}

Composite distributions of minimum surface distances calculated between

contours propagated using original and synthetic displacement vector fields for cord, esophagus, heart, and lung contours. 

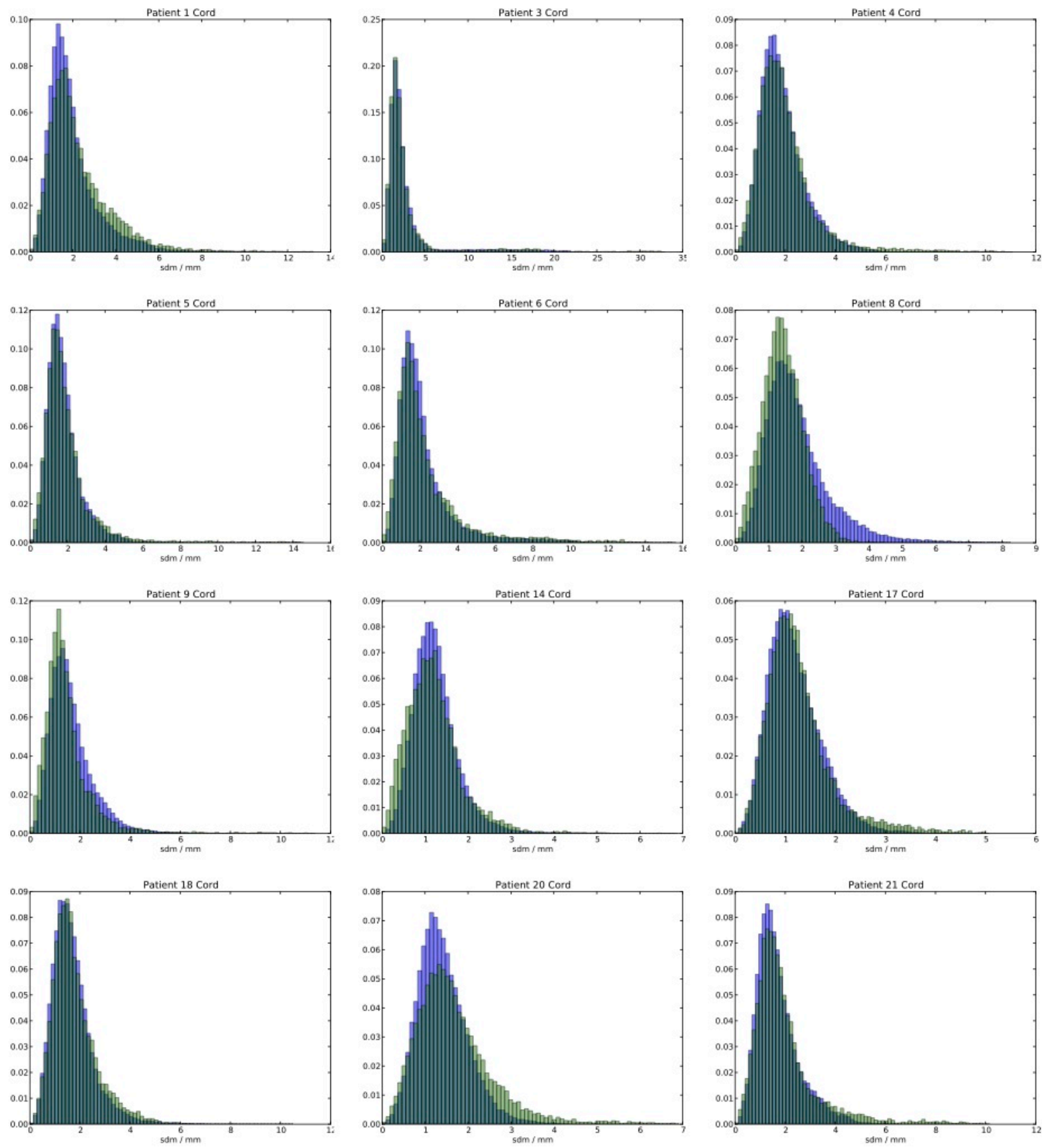

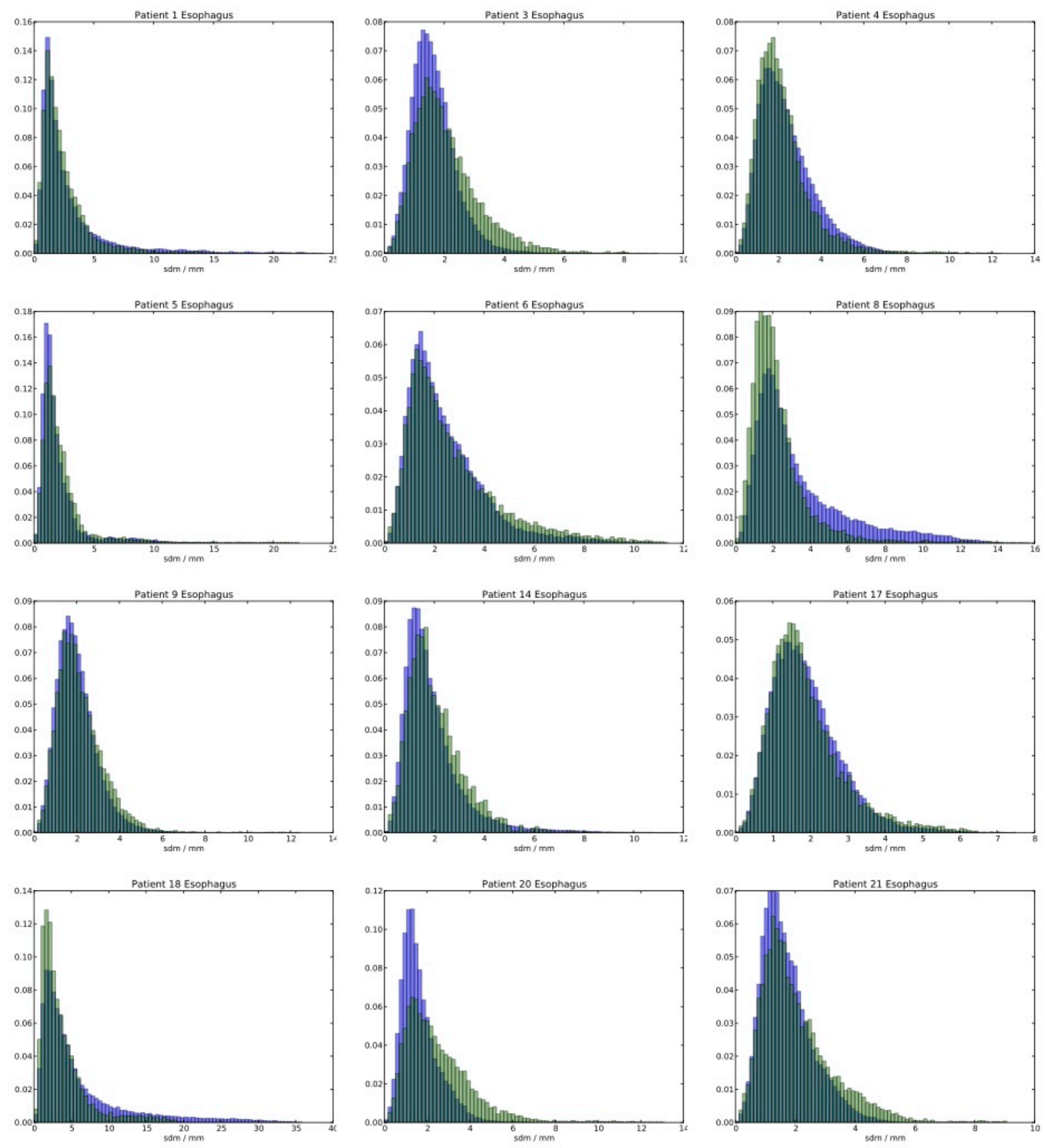

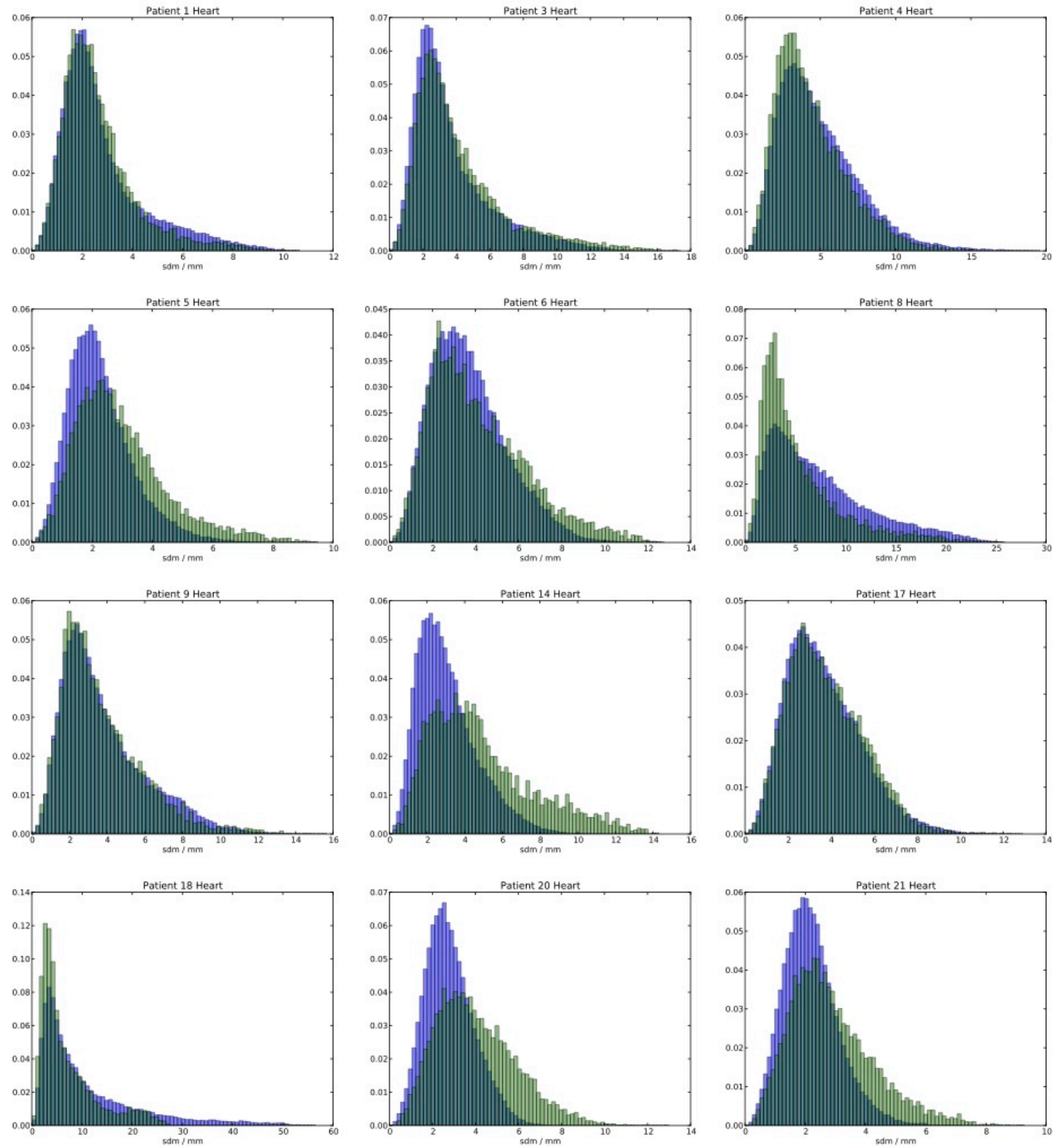

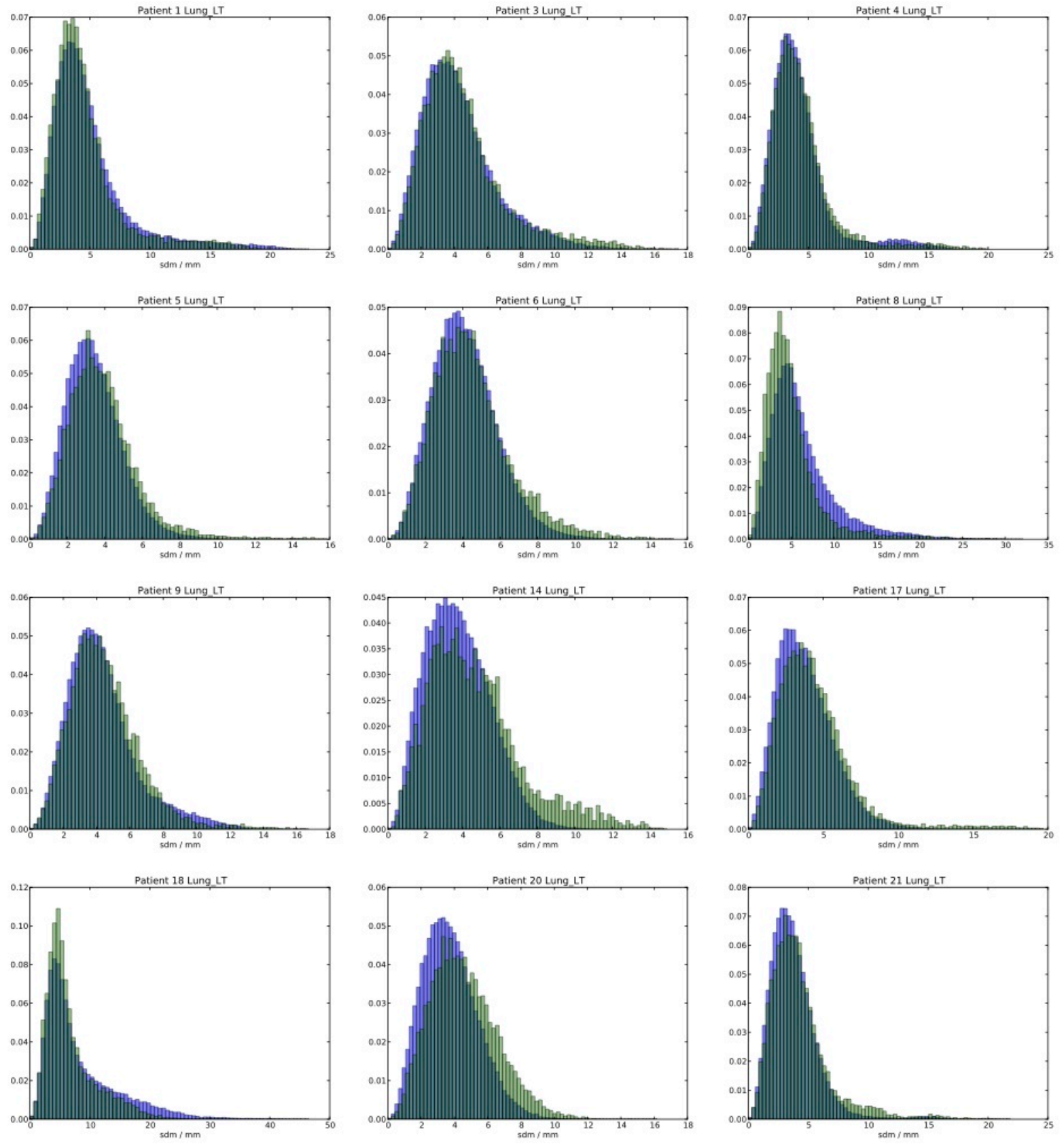

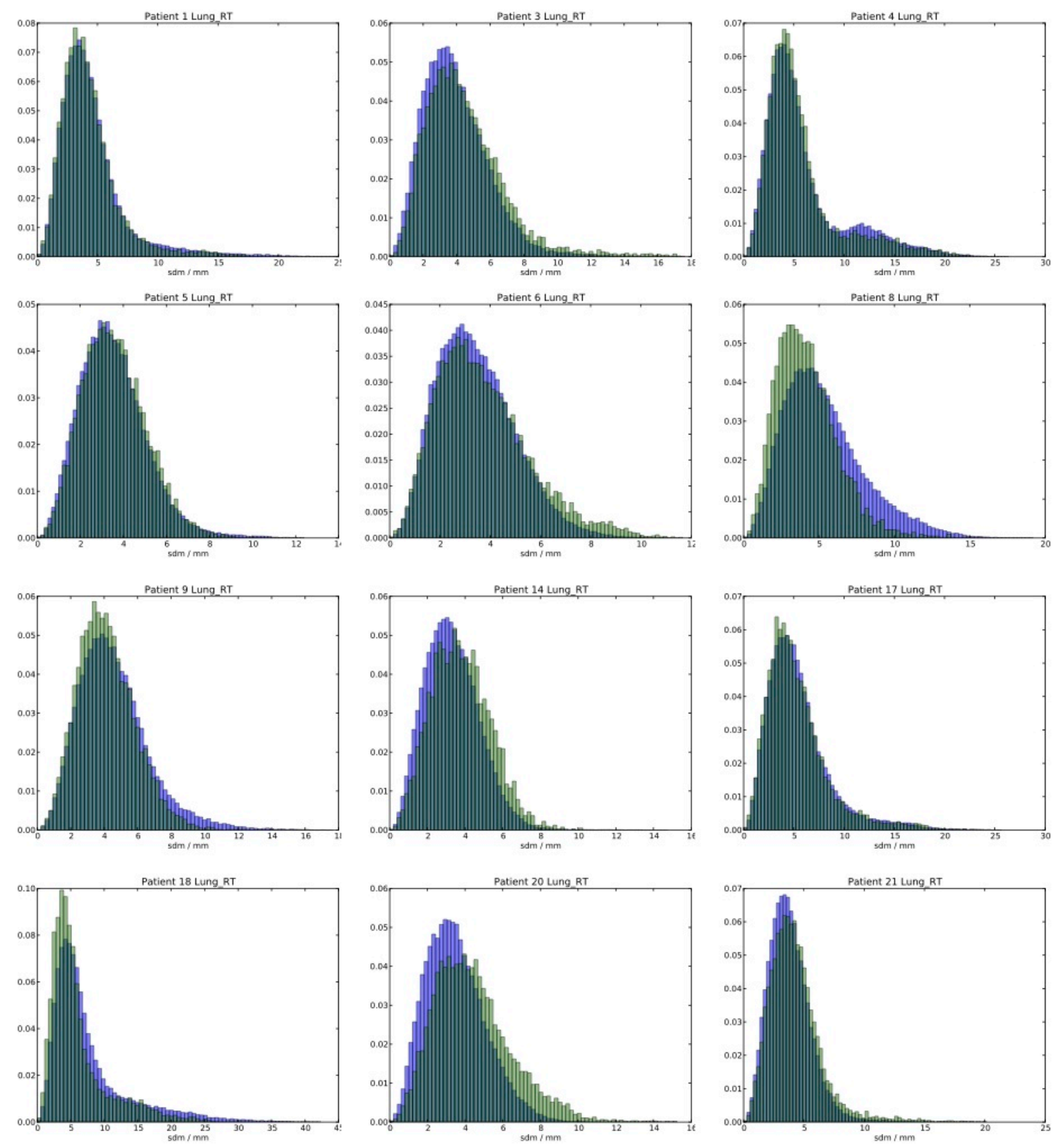


\section{Appendix VI}

Adaptive therapy study additional data: dose volume histograms 

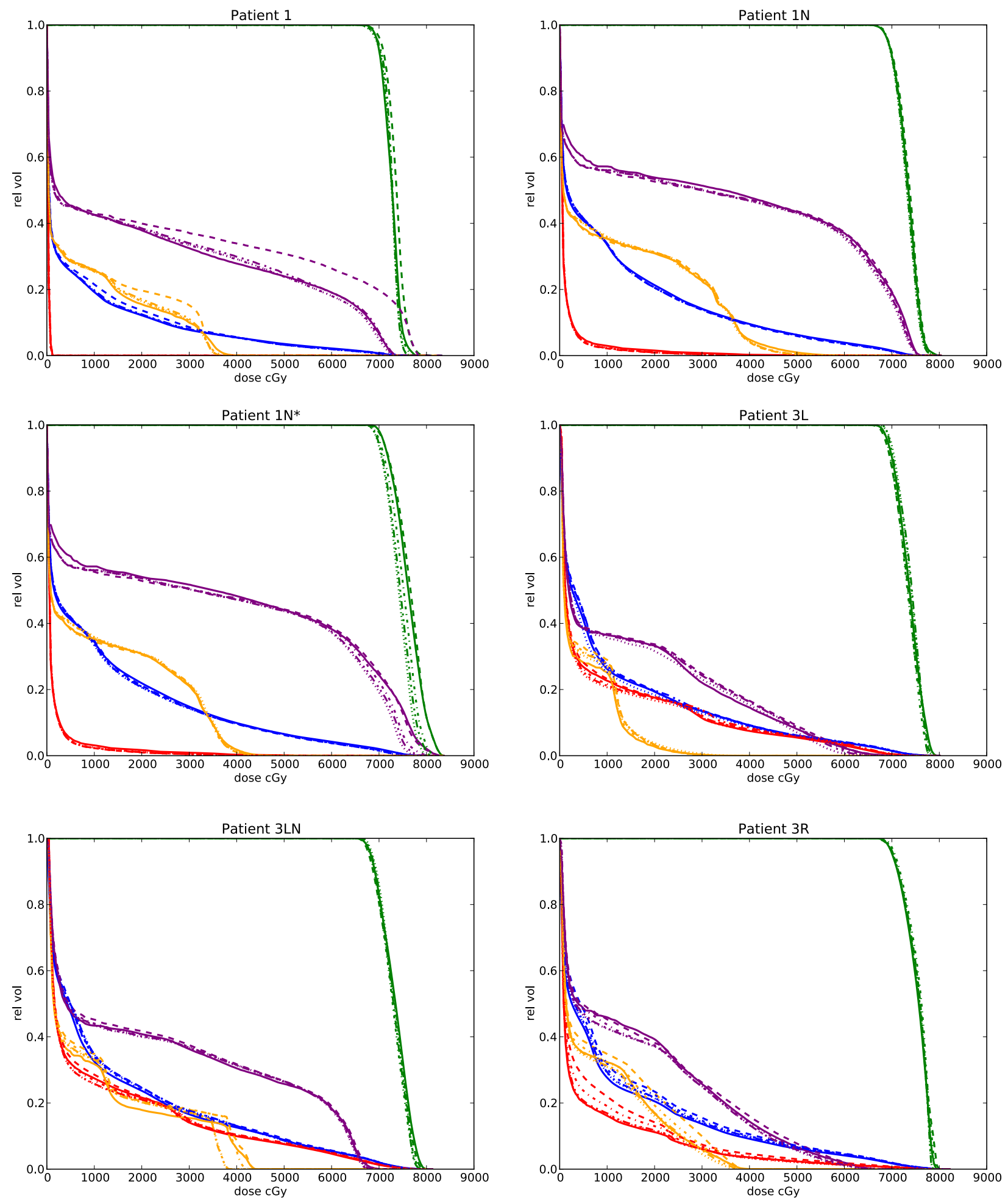

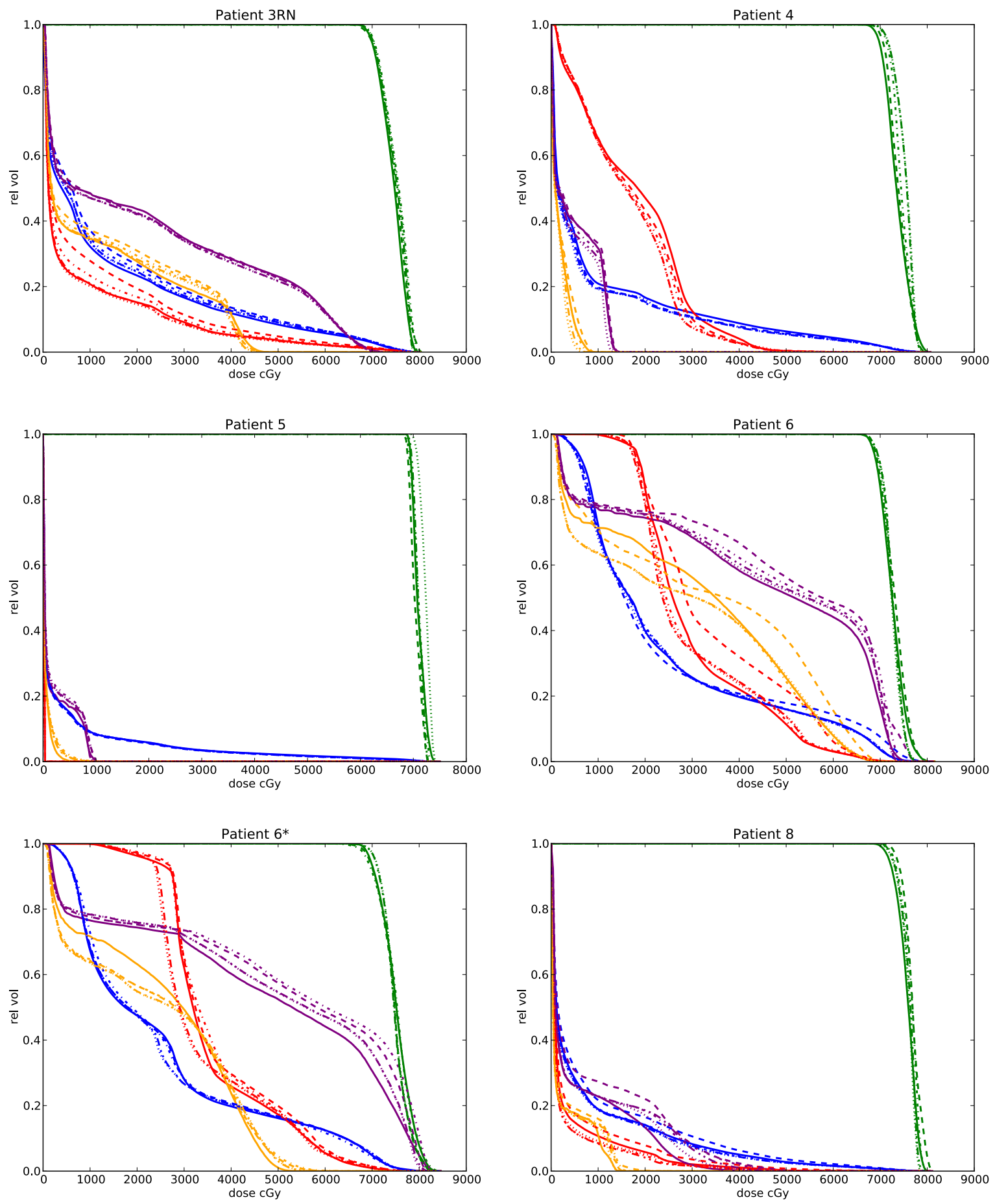

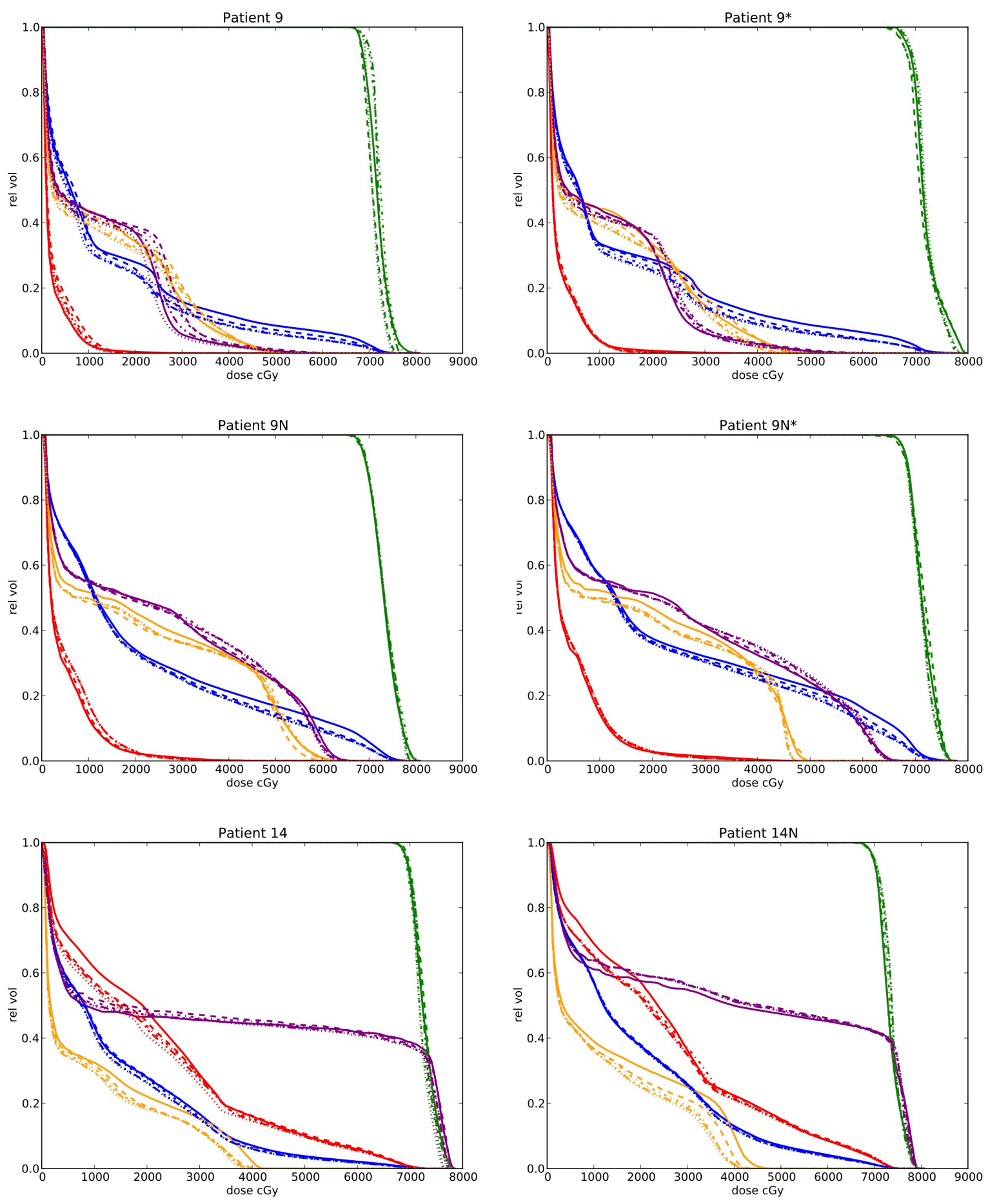

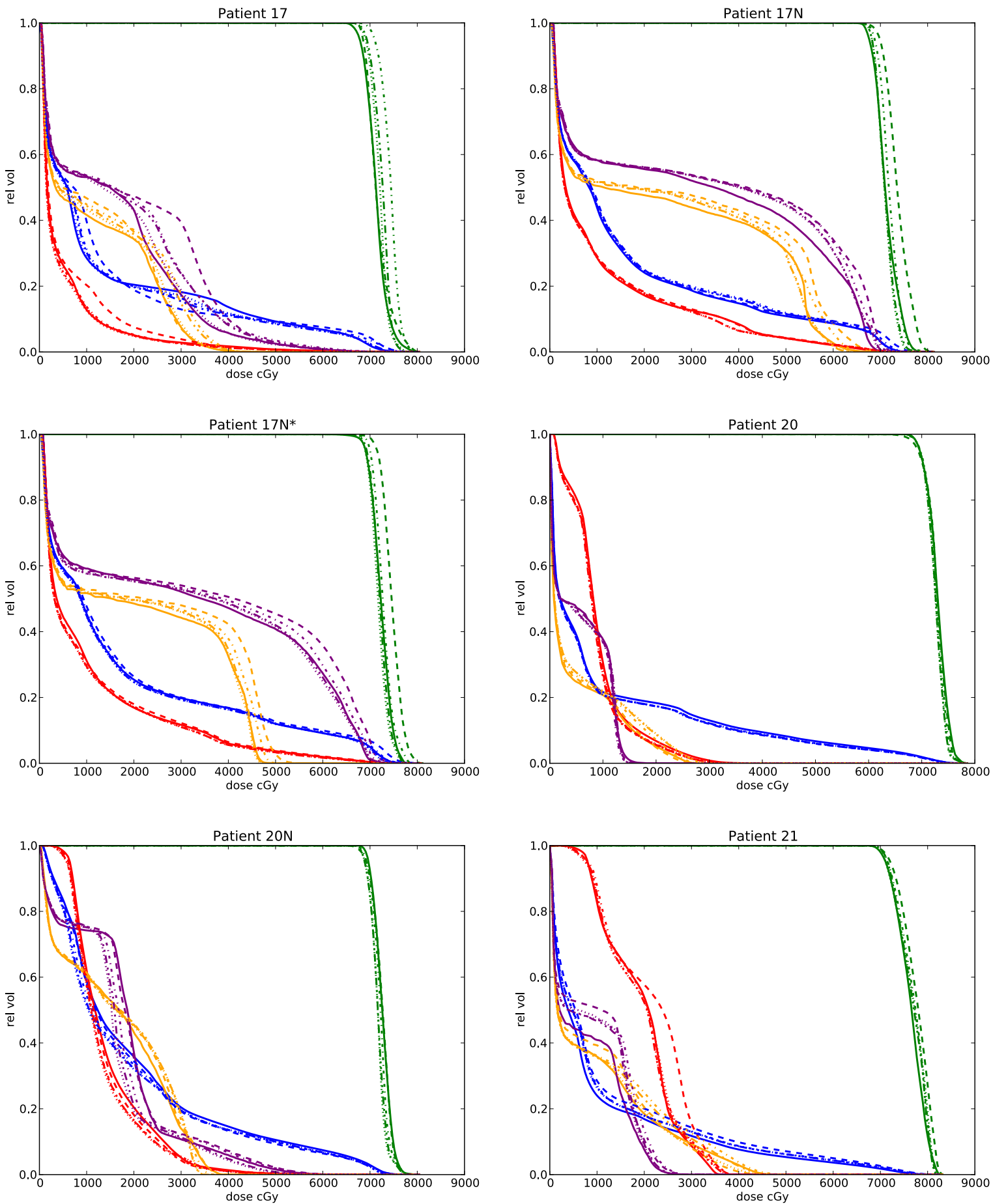


\section{Vita}

Christian Dial was born in Salt Lake City, Utah on November $14^{\text {th }} 1979$. He attended West High School in Salt Lake City and graduated in 1998. After graduation, Christian spent two years in the western region of Guatemala as a missionary for his church and returned home to Utah in December of 2000. After some initial years at the University of Utah in premedical studies, he declared his major in Physics and graduated with a Bachelor of Science degree in the spring of 2008. Upon completion of his undergraduate degree, he spent a year as a research assistant with the Utah Center for Advanced Imaging Research (UCAIR). Christian enrolled at Virginia Commonwealth University in the fall of 2009 and began research in the fall of 2010 under the direction of Drs. Geoffrey Hugo and Jeffrey Siebers. 